SCOTT, James Floyd, 1942INFRARED ABSORPTION BANDS OF ACETYLENE.

The Ohio State University, Ph.D., 1966

Physics, general

University Microfilms, Inc., Ann Arbor, Michigan 


\title{
INFRARED ABSORPTION BANDS OF ACETYLENE
}

\author{
DISSERTATION \\ Presented in Partial Fulfillment of the Requirements for the \\ Degree Doctor of Philosophy in the Graduate School of \\ The Ohio State University
}

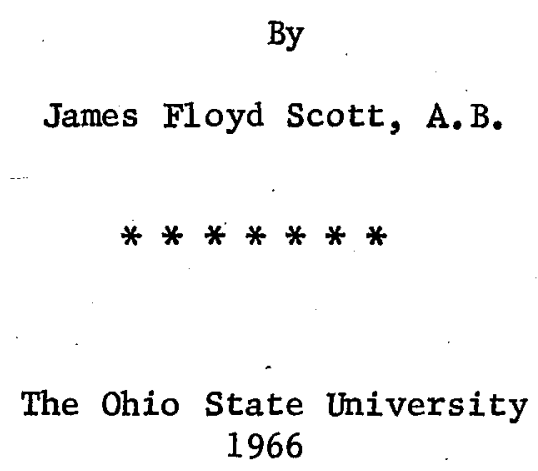

Approved by

K. havahavi Rao

Adviser

Department of Physics 


\section{ACKNOWLEDGMENTS}

I would like to thank most sincérely Professor $K$. Narahari Rao, who taught me what spectroscopy is, Professor Harald H. Nielsen, who taught me why some things are as they are, and my wife Carol, who put up with me during the learning period.

I wish to acknowledge also the financial assistance provided through a National Science Foundation Summer Fellowship for Graduate Teaching Assistants, a National Aeronautics and Space Administration Traineeship, and teaching and research assistantships in the Department of Physics.

Finally, I want to thank my fellow graduate students and laboratory coworkers for their many helps, especially m. Jan Adams, who performed most of the numerical calculations on the university's IBM 7094. 
VITA

$\begin{array}{ll}\text { May 4, } 1942 \text { Born - Beverly, New Jersey } \\ 1963 & \text { A.B., Harvard University, Cambridge, Massachusetts } \\ 1963-1964 & \begin{array}{l}\text { Teaching Assistant, Department of Physics, } \\ \text { The Ohio State University, Columbus, Ohio }\end{array} \\ 1964 & \begin{array}{l}\text { National Science Foundation Sumer Fellow, } \\ \text { The Ohio State University, Columbus, Ohio }\end{array} \\ 1964-1965 & \begin{array}{l}\text { Research Assistant, Department of Physics, } \\ \text { The Ohio State University, Columbus, Ohio }\end{array} \\ 1965-1966 & \text { National Aeronautics and Space Administration, } \\ & \text { Trainee, The Ohio State University, Columbus, Ohio }\end{array}$

PUBLICATIONS

J. F. Scott and R. Narahari Rao, "Infrared Absorption Bands of Acetylene. Part I. Analysis of the Bands at 13.7 Microns," J. Mol. Spectry. $16,15 \cdot(1965)$.

J. F. Scott and K. Narahari Rao, "Infrared Bands of Acetylene," contributed paper R7, Symposium on Molecular Structure and Spectroscopy, The Ohio State University, Columbus, Ohio, June 16, 1965.

J. F. Scott and K. Narahari Rao, "Infrared Absorption Bands of Acetylene. Part II. The Effect of Vibrational l-type Doubling," J. Mo1. Spectry. 18, 152 (1965).

J. F. Scott and $\mathrm{K}$. Narahari Rao, "Current Trends in High Resolution Infrared Spectroscopic Research," contributed paper B13, meeting of the Ohio Section of the American Physical Society, Dayton, Ohio, November 13, 1965.

J. F. Scott and K. Narahari Rao, "Infrared Absorption Bands of Acetylene. Part III. Analysis of the Bands at 3 Microns," J. Mo1. Spectry. (in press).

J. F. Scott and K. Narahari Rao, "Use of Loomis-Wood Plots in Vibration-Rotation Analysis," J. Mo1. Spectry. (in press). 
CONTENTS

Chapter Page

I. INTRODUCTION . . . . . . . . . . 1

II. ANALYSIS OF THE BANDS AT 13.7 MICRONS . . . . 7

III. VIBRATIONAL l-TYPE RESONANCE AND DOUBLING . • 27

IV. ANALYSIS OF THE BANDS AT 3 MICRONS ...... 53

V. THE USE OF LOOMIS-WOOD DIAGRAMS . . . . . 97

VI. BANDS IN THE PHOTOGRAPHIC INERARED ...... 105 APPENDIX ....................... 122

REFERENCES .................. 141 


\section{LIST OF TABLES}

Table

Page

1. Vacuum Wave Numbers $\left(\mathrm{cm}^{-1}\right)$ of the Rotational Lines of Acetylene - $v_{5}$ fundamental of ${ }^{12} \mathrm{C}_{2} \mathrm{H}_{2}$...... 15

2. Vacuum Wave Numbers $\left(\mathrm{cm}^{-1}\right)$ of the Rotational lines of Acetylene $-v_{5}$ fundamental of ${ }^{12} \mathrm{c}^{13} \mathrm{CH}_{2}$..... 16

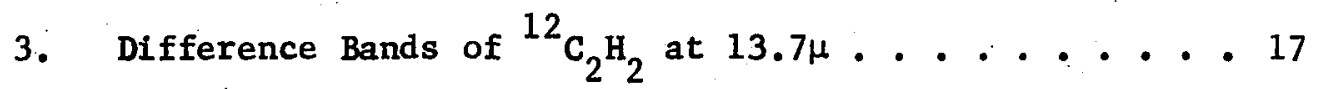

4. Some of the Band Centers and Rotational Constants of

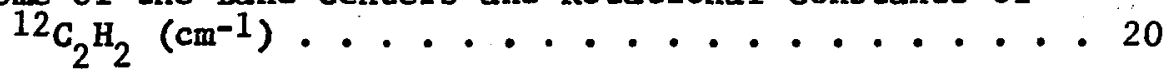

5. Vibrational Levels of Acetylene $\left(\mathrm{cm}^{-1}\right) . . . . . .21$

6. Vibrational Interaction Constants of ${ }^{12} \mathrm{C}_{2} \mathrm{H}_{2}$. . . . 48

7. Transitions Observed at Three Microns . . . . . . 58

8. Rotational Analysis of the Bands $v_{3}$ and $v_{2}+v_{4}+v_{5}$
$\left(\mathrm{~cm}^{-1}\right)$. . . . . . . . . . . . . . .

9. Constants Derived from the Bands at 3 Microns . . . 66

10. Rotational Analysis of the Band $v_{3}+v_{4}-v_{4}$.... 82

11. Rotational Analysis of the Band $v_{3}+v_{5}-v_{5}$.... 84

12. Rotational Analysis of the Band

$$
v_{2}+2 v_{4}^{2}+v_{5}^{1}-v_{4}^{1} \text {............. } 87
$$

13. Rotational Analysis of the Band

$$
v_{2}+v_{4}^{1}+2 v_{5}^{2}-v_{5}^{1} \text {............. } 90
$$

14. Line Positions of Some Photographic Bands . . . . . 108

15. Rotation Constants Derived from the Photographic

Bands .................. 116 


\section{ILLUSTRATIONS}

Matrices

Page

I. Vibration Rotation Submatrix Pertaining to the Levels 00011 . . . . . . . . . 30

II. Vibration Submatrix Pertaining to 00011. . . . . . 32

III. Vibration Rotation Submatrix Pertaining to the Levels 00021 . . . . . . . . . . 34

IV. Vibration Submatrix Pertaining to 00021 ..... 36

V. Vibration Rotation Submatrix Pertaining to the Levels 00031 . . . . . . . . . 38

VI. Vibration Submatrix Pertaining to 00031. . . . . 40

\section{Figures}

1. Vibration Rotation Bands of Acetylene at 13.7 Microns 10

2. Vibration Rotation Bands of Acetylene at 13.7 Microns : 12

3. Vibration Rotation Bands of Acetylene at 13.7 Microns 14

4. The Energy Levels 00011 and 00021. . . . . . . . . 42

5. The Energy Levels 00031 . . . . . . . . . . 44

6. An Energy Level Diagram of ${ }^{12} \mathrm{C}_{2} \mathrm{H}_{2}$. . . . . . . . 50

7. The Spectrum at 3 Microns ............ 55

8. Transitions Expected at 3 Microns ... . . . . . . 57

9. $\quad v(\mathrm{~m})$ versus $\mathrm{m}$ for $v_{2}+v_{4}+v_{5} \ldots \ldots . . . . . .69$

10. $\mathrm{R}(\mathrm{J}-1)+\mathrm{P}(\mathrm{J})$ versus $\mathrm{J}$ for $v_{3} \ldots \ldots . . . . . . .71$ 


\section{IILUSTRATIONS (Continued)}

Figures $\quad: \quad$ Page

11. Submatrix Pertaining to Transitions at 3 Microns . . 74

12. Graphical Determination of Rotational $\ell$-type

Doubling Constants . . . . . . . . . . 96

13. Loomis-Wood Diagram of the Spectrum at 13.7 Microns. . 100

14. Loomis-Wood Diagram of the Spectrum at 3 Microns . . 102 . 


\section{INTRODUCTION}

In the spectroscopic study of molecules it is customary to measure the positions of observed transitions and to determine from these transitions a set of energy levels compatible with the observations.

The energy levels are known to fit a particular functional dependence, since they are eigenvalues of the Hamiltonian for the molecule, and the eigenvalue problems for most molecular geometries have been solved to a high order of approximation. The experimentaliy determined energy levels are then used to fit the constants which occur in the general eigenvalue expression. The infrared region of the spectrum is most useful for the determination of constants of the energy eigenvalue expression which are associated with rotation and vibration of the molecule.

The infrared absorption spectrum of the acetylene molecule, $\mathrm{HCCH}$, has been analyzed by a large number of investigators from many different laboratories over the past forty years. As a result, quite a lot is known about the molecule; it is 1 inear and symmetric with respect to inversion (1), and its fundamentals have all been unambiguously assigned, although the asymmetric stretch had been misassigned in the literature up until 1964 (2). While many bands have been assigned with certainty, the basic problem of determining a set of 
vibrational and rotational constants which will mathematically reproduce the observed spectrum to within the experimental uncertainty remains today.

To a very large extent this difficulty is due to what are loosely termed "resonances," that is, to non-negligible off-diagonal elements in the Hamiltonian, which complicate the eigenvalue problem. Another reason for the difficulty encountered in deriving a good set of vibrational constants for acetylene is the fact that the molecule has a high degree of symmetry, making electric dipole transitions from the ground state to many of the vibrational energy levels forbidden by selection rules, so that many levels have not yet been determined from observed transitions. In addition, the large number of energy levels determined from transitions observed in the visible and photographic infrared regions of the spectrum have been heretofore unusable for determining vibrational constants for the reason that these levels have been thought to involve such high quantum numbers that the cubic terms in the vibrational energy expression, which are negligible in the infrared region, apparently become sizeable. This leads to difficulty since the experimentally determined energy levels yield equations which can be solved:simultaneously with vibrational constants as unknowns; if cubic terms must be included, the number of unknowns becomes larger for this molecule than the number of available energylevel equations, thus precluding solution for the unknowns. It has been my aim in the present study to examine several selected regions of the acetylene spectrum in which one could expect to find transitions involving levels which had not previously been 
observed. In particular, analysis has been made of regions of the spectrum in which discrepancies were apparent (3) between observed and calculated vibrational'transitions. It is gratifying that in each case considered in the present study, agreement has been obtained between the observed and calculated positions of all the energy levels involved. In one case (4) the assignment of a Q-branch by a previous investigator was a simple error; the transition was thought to have lower state $0000^{0} 1^{1}$ but in fact has lower state $0001^{1} 0^{0}$. In the three micron region it was found (5) that the inclusion of the effect of vibrational -type resonance (6), previously neglected by investigators of this region, brings about good numerical agreement of the vibrational levels involved in transitions there. Two bands at about five microns have also been reassigned on this basis. It should be emphasized that the discrepancies in the acetylene spectrum which are reconciled here were not completely ignored by previous workers; it is just that they were unable to treat the problems quantitatively because of their lack of information about certain vibrational levels. The necessary information on levels of interest has been obtained by the analysis of overlapping bands in the 3 and 13.7 micron regions. In the case of the 13.7 micron bands, analysis was made of what apparently is better data than previously obtained for this region of the acetylene spectrum. In the case of the 3 micron region, however, since very recent investigations of this region have been made (2) at the National Bureau of Standards with resolution at least. as good as that obtained here, the success in analyzing the spectra 
must be attributed to the development and use of the diagram techniques discussed in Chapter V.

In both the 3 and 13.7 micron regions primary interest was in obtaining information about the vibrational constants which are involved in the energy expression when both degenerate modes of the molecule are excited. Previously it had been observed that the only numerical values obtainable for these constants led to inconsistencies (3). The history of the investigation of these constants is very curious. Since 1955, due to Keller's work (7), the diagonal terms of the Hamiltonian for levels involving only the two degenerate modes have been expressed by Eq. (1) below:

$$
\begin{aligned}
G_{0}(v)=w_{4}^{0} v_{4}+w_{5}^{0} v_{5} & +x_{44} v_{4}^{2}+x_{45} v_{4} v_{5}+x_{55} v_{5}^{2} \\
& +g_{44} \ell_{4}^{2}+g_{45} l_{4} l_{5}+g_{55} \ell_{5}^{2}
\end{aligned}
$$

Previous to Keller's work it was thought, for theoretical reasons, that the constant $g_{45}$ above must be zero (8). His experimental work indicated that the observed energy levels -- that is, energy levels determined directly from observed transitions -- could not be mathematically reproduced without the inclusion of a non-zero $g_{45}$. Subsequent theoretical reconsiderations showed that $\mathrm{g}_{45}$, was, indeed, non-zero. Information about non-zero off-diagonal terms arising from the theoretical reconsiderations of the acetylene molecule has also proved important. Eq. (2) below is an expression for an important Fermi 
resonance, important because for several pairs of leve1s, 1ike $0010^{0} 0^{0}$ and $0101^{1} 1^{1}$, the interaction term is larger than the separation of the unperturbed levels, so that a substantial mixing of the wave functions occurs :

$$
\begin{gathered}
\left(\mathrm{v}_{1} ; \mathrm{v}_{2} ; \mathrm{v}_{3} ; \mathrm{v}_{4}, \ell_{4} ; \mathrm{v}_{5}, \ell_{5}|\mathrm{H}| \mathrm{v}_{1} ; \mathrm{v}_{2}-1 ; \mathrm{v}_{3}+1 ; \mathrm{v}_{4}-1, \ell_{4} \mp .1 ;\right. \\
\left.\mathrm{v}_{5}-1, \ell_{5} \pm 1\right)=(-\gamma / 8)\left[\mathrm{v}_{2}\left(\mathrm{v}_{3}^{-}+1\right)\left(\mathrm{v}_{4} \pm \ell_{4}\right)\left(\mathrm{v}_{5} \mp \ell_{5}\right)\right]^{1 / 2}
\end{gathered}
$$

Eq. (3) is an expression for the terms resulting in what has been called "vibrational $\ell$-type resonance" and "vibrational $\ell$-type doubling." Physically, this expression reflects the fact that there are terms which are diagonal in the total vibrational angular momentum, but non-diagonal in the vibrational angular momenta of the individual degenerate modes. In Eqs. (1-3) the usual notation for vibrational states has been employed; $v_{i}$ refers to the principal vibrational quantum number of the -i-th normal mode of vibration, $i=1,2,3,4,5 ; l_{i}$ refers to the vibrational angular momentum introduced about the figure axis by the i-th transverse mode, $i=4,5$. States are designated by the notation $v_{1} v_{2} v_{3} v_{4}^{l_{4}} v_{5}^{l_{5}}$, so that $0101^{1} 1^{1}$ is the state in which $v_{1}=0$, $\mathrm{v}_{2}=1, \mathrm{v}_{3}=0, \mathrm{v}_{4}=1, \ell_{4}=1, \mathrm{v}_{5}=1$, and $\ell_{5}=1$. It is sometimes desirable to distinguish between such states by the total $\ell$-value; hence $0101^{ \pm 1} 1^{\mp 1}$ is a sigma-state, having a total vibrational angular momentum equal to zero, while $0101^{ \pm 1} 1_{1}$ is a delta-state, having a total vibration al angular momentum of magnitude 2 (units are $\hbar$ ). 


$$
\begin{aligned}
& \left(\mathrm{v}_{4}, \ell_{4} ; \mathrm{v}_{5}, l_{5}\left|\mathrm{H}_{2}^{\prime}\right| \mathrm{v}_{4}, \ell_{4} \pm 2 ; \mathrm{v}_{5}, l_{5} \mp 2\right)= \\
& 1 / 4 \mathrm{r}_{45}\left[\left(\mathrm{v}_{4} \pm \ell_{4}+2\right)\left(\mathrm{v}_{4} \mp \ell_{4}\right)\left(\mathrm{v}_{5} \mp l_{5}+2\right)\left(\mathrm{v}_{5} \pm l_{5}\right)\right]^{1 / 2}
\end{aligned}
$$

The present work concerns itself in part with the evaluation of the constants appearing in Eqs. $(1-3)$. It is the first such attempt known to the author to include the effect of the terms represented by Eq. (3). The inclusion of this effect has led to strikingly different values for the constants $x_{45}$ and $g_{45}$ of Eq. (1) and is discussed in detail in Chapter III. 


\section{CHAPTER II}

\section{ANALYSIS OF THE BANDS AT 13.7 MICRONS}

High resolution infrared spectroscopists have studied many of the vibration rotation bands of the acetylene molecule, and recently Plyler, Tidwell, and Wiggins (3) described an attempt to correlate all the available data for determining a set of vibrational constants for this molecule. In this attempt, these authors encountered some difficulties. In particular, their vibrational constants do not predict the reported (7) measurement for the $0000^{0} 2^{2}$ state of ${ }^{12} \mathrm{C}_{2} \mathrm{H}_{2}$ any closer than $2 \mathrm{~cm}^{-1}$. Therefore, it seemed that measurement and analysis of the absorption bands of acetylene observed at $13.7 \mu$ might provide answers to some of the questions raised by Plyler, Tidwel1, and Wiggins, because in this spectral region it is possible to observe not only the $v_{5}$ fundamental vibration rotation band of this molecule (transition $0000^{0} 1^{1} \leftarrow 0000^{0} 0^{0}$ ) but also several "difference" bands including the $2 v_{5}{ }^{2}-v_{5}$ band (transition $0000^{0} 2^{2} \leftarrow 0000^{0} 1^{1}$ ). In fact, the observed rotational structure has been assigned to five bands of ${ }^{12} \mathrm{C}_{2} \mathrm{H}_{2}$ and one $\left(v_{5}\right.$ fundamental $)$ of ${ }^{12} \mathrm{C}^{13} \mathrm{CH}_{2}$. 


\section{EXPERIMENTAL DETAIIS}

The observational data were obtained by employing a vacuum infrared grating spectrograph. Figs. (1-3) show a tracing of the observed spectrum along with the analysis, and Tables 1, 2, and 3 give vacuum wave numbers $\left(\mathrm{cm}^{-1}\right)$ of the rotational lines measured for the different bands observed. It may be noted that the previous assignment (7) for the $2 v_{5}^{2}-v_{5}^{1}$ band has been revised and it has been shown to be the $\left(v_{4}+v_{5}\right)^{2}-v_{4}^{1}$ band. A different set of rotational lines has been identified as belonging to the $2 v_{5}^{2}-v_{5}^{1}$ band.

The data shown in Figs. (1-3) were taken with the Pfund-type spectrograph in our laboratory. The absorption pathlength was two meters; the pressure of the gas was one centimeter of mercury; the detector employed was a thermocouple. Some unassigned rotational lines are present, particularly around $725 \mathrm{~cm}^{-1}$. The line at $711 \mathrm{~cm}^{-1}$ indicated by an arrow is probably the Q-branch of a transition having the lower state $0002^{0} 0^{0}$ or $0002^{2} 0^{0}$; its assignment remains uncertain.

\section{MOLECULAR CONSTANTS OF ACETYLENE DERIVED FROM THE PRESENT STUDY}

Band Centers and Rotational Constants:

Standard techniques (1) have been employed in determining the band centers and rotational constants from the observational data presented in Tables $1-3$, and the values are summarized in Table 4.

A few comments are of interest regarding the distortion constants of some of the levels of acetylene involved here. Coburn, Rao, and Nielsen (9) have already reported the anomalous nature of the distortion constants evaluated from the observational data pertaining 
Figure 1

Vibration Rotation Bands of Acetylene at 13.7 Microns 


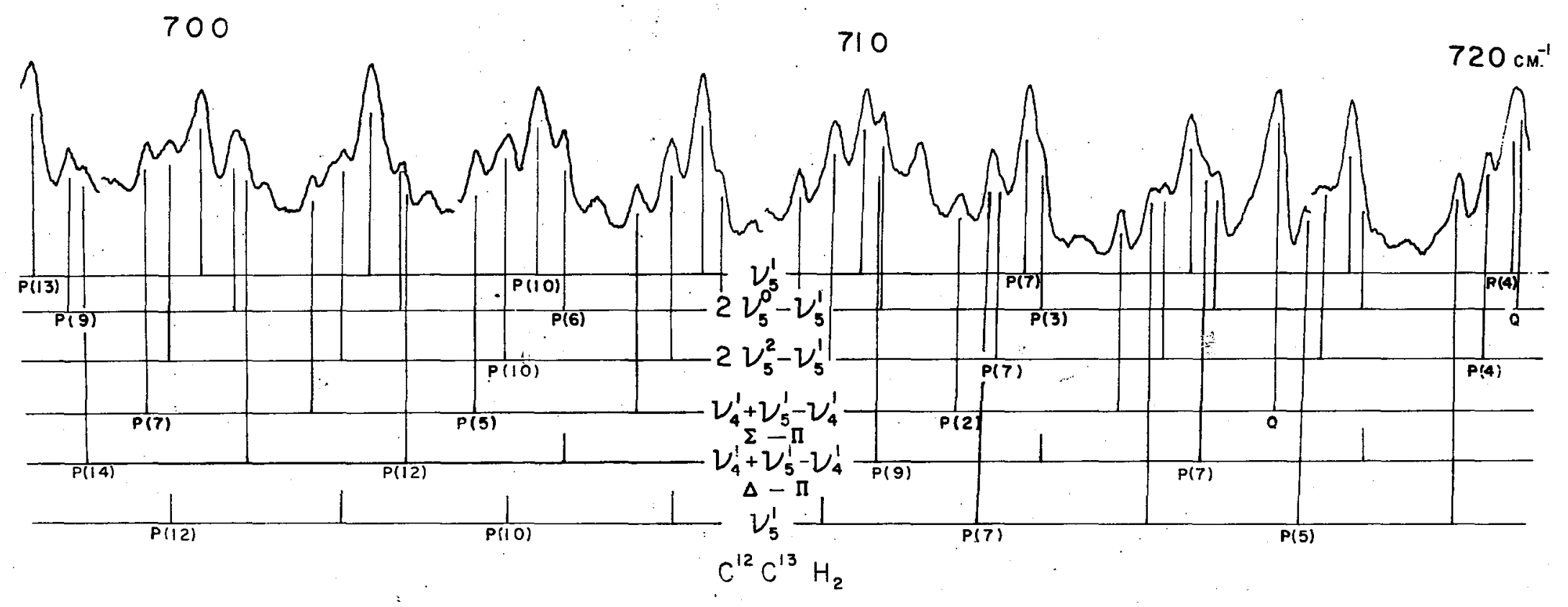


Figure 2

Vibration Rotation Bands of Acetylene at 13.7 Microns 


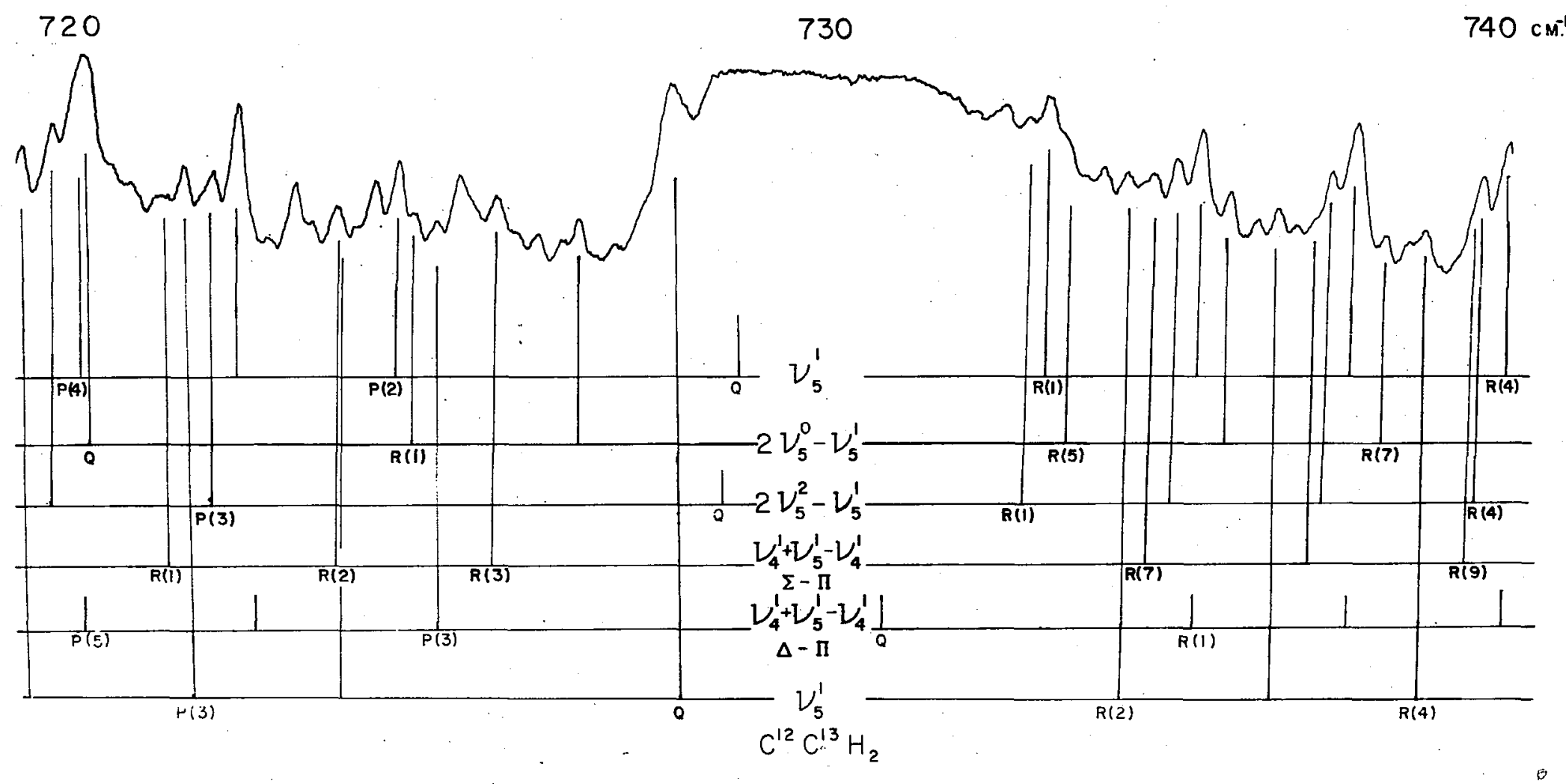




\section{Figure 3}

Vibration Rotation Bands of Acetylene at 13.7 Microns 
740

750

760

$\mathrm{cm}^{-1}$

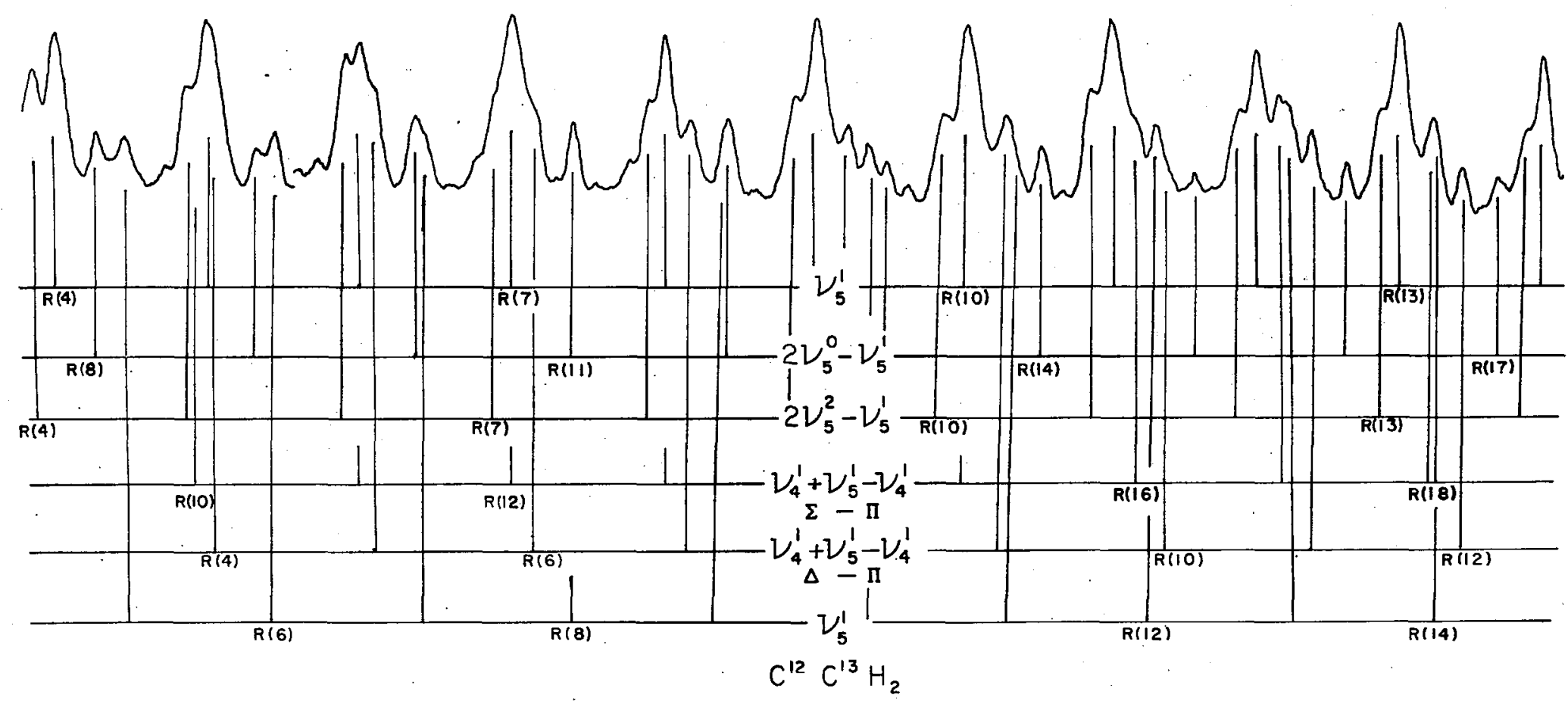


TABLE 1

VACUUM WAVE NUMBERS $\left(\mathrm{cm}^{-1}\right)$ OF THE ROTATIONAL LINES OF ACETYLENE

$v_{5}$ fundamental of ${ }^{12} \mathrm{C}_{2} \mathrm{H}_{2}$

\begin{tabular}{|c|c|c|c|c|c|}
\hline $\mathbf{J}$ & $\mathrm{R}(\mathrm{J})$ & $P(J)$ & $\mathbf{J}$ & $R(J)$ & $P(J)$ \\
\hline 0 & & & 24 & $787: 71$ & 672.67 \\
\hline 1 & 733.86 & & 25 & 790.07 & 670.32 \\
\hline 2 & 736.26 & 724.45 & 26 & 792.40 & 668.00 \\
\hline 3 & 738.60 & 722.10 & 27 & 794.69 & 665.58 \\
\hline 4 & 740.93 & 719.78 & 28 & 797.01 & 663.24 \\
\hline 5 & 743.27 & 717.38 & 29 & 799.37 & 660.93 \\
\hline 6 & 745.52 & 715.03 & 30 & 801.65 & 658.58 \\
\hline 7 & 747.91 & 712.68 & 31 & 803.99 & 646.21 \\
\hline 8 & 750.28 & 710.32 & 32 & 806.29 & 653.84 \\
\hline 9 & 752.66 & 707.95 & 33 & 808.62 & 651.50 \\
\hline 10 & 755.05 & 705.61 & 34 & 810.91 & 649.20 \\
\hline 11 & 757.33 & 703.24 & 35 & 813.23 & 646.83 \\
\hline 12 & 759.70 & 700.88 & 36 & 815.57 & 644.48 \\
\hline 13 & 762.02 & 698.53 & 37 & 817.86 & 642.14 \\
\hline 14 & 764.34 & 696.17 & 38 & 820.15 & 639.78 \\
\hline 15 & 766.72 & 693.72 & 39 & 822.44 & 637.47 \\
\hline 16 & 769.06 & 691.46 & 40 & 824.75 & 635.13 \\
\hline 17 & 771.41 & 689.10 & 41 & 827.02 & 632.76 \\
\hline 18 & 773.74 & 686.75 & 42 & 829.17 & 630.47 \\
\hline 19 & 776.07 & 684.44 & 43 & 831.65 & 628.10 \\
\hline 20 & 778.42 & 682.10 & 44 & 833.91 & 625.74 \\
\hline 21 & 780.77 & 679.73 & $45^{\prime}$ & 836.21 & 623.45 \\
\hline 22 & 783.07 & 677.35 & 46 & 838.47 & \\
\hline 23 & 785.39 & 675.01 & & & \\
\hline
\end{tabular}


TABLE 2

VACUUM WAVE NUMBERS $\left(\mathrm{cm}^{-1}\right)$ OF THE ROTATIONAL

LINES OF ACETYLENE

\begin{tabular}{|c|c|c|c|c|c|}
\hline $\mathrm{J}$ & $\mathbf{R}(\mathrm{J})$ & $P(J)$ & $\mathbf{J}^{\circ}$ & $R(J)$ & $P(J)$ \\
\hline 0 & & & 14 & 762.60 & 696.17 \\
\hline 1 & & & 15 & 764.95 & 693.90 \\
\hline 2 & 735.13 & 723.57 & 16 & 767.13 & \\
\hline 3 & 737.46 & 721.34 & 17 & 769.50 & \\
\hline 4 & 739.71 & 719.00 & 18 & & 687.28 \\
\hline 5 & 741.99 & 716.77 & 19 & & 684.94 \\
\hline 6 & 744.28 & 714.53 & 20 & & 682.74 \\
\hline 7 & 746.45 & 712.18 & 21 & & 680.41 \\
\hline 8 & 748.85 & 709.98 & 22 . & & 678.24 \\
\hline 9 & 751.25 & 707.52 & 23 & & 676.00 \\
\hline 10 & 753.20 & 705.21 & 24 & & 673.78 \\
\hline 11 & & 702.89 & 25 & & 670.55 \\
\hline 12 & $\cdot$ & 700.64 & 26 & & 668.29 \\
\hline 13 & & 698.39 & & & \\
\hline
\end{tabular}


TABLE 3

DIFFERENCE BANDS OF ${ }^{12} \mathrm{C}_{2} \mathrm{H}_{2}$ AT $13.7 \mu$

\begin{tabular}{|c|c|c|c|c|c|c|c|c|}
\hline \multirow[b]{2}{*}{$\mathrm{J}$} & \multicolumn{2}{|c|}{$\begin{array}{c}2 v_{5}^{0}-v_{5} \\
\Sigma-\Pi\end{array}$} & \multicolumn{2}{|c|}{$\begin{array}{c}2 v_{5}^{2}-v_{5} \\
\Delta-\Pi\end{array}$} & \multicolumn{2}{|c|}{$\left(v_{4}+v_{5}\right)^{0}-v_{4}$} & \multicolumn{2}{|c|}{$\left(v_{4}+v_{.5}\right)^{2}-v_{4}$} \\
\hline & $R(J)$ & $P(J)$ & $R(J)$ & $P(J)$ & $R(J)$ & $P(J)$ & $R(J)$ & $P(J)$ \\
\hline 0 & & & & & & & & \\
\hline 1 & 724.70 & 717.60 & 733.61 & & 721.06 & 714.04 & & \\
\hline 2 & 727.09 & 715.34 & 735.88 & $\theta$ & 723.57 & 711.72 & & \\
\hline 3 & & 712.88 & 738.24 & 721.77 & 726.00 & 709.39 & & 725.02 \\
\hline 4 & & 710.57 & 740.57 & 719.43 & & 707.04 & 1 & \\
\hline 5 & 734.31 & 708.24 & 742.93 & 716.97 & & 704.76 & 745.85 & \\
\hline 6 & 736.72 & 705.98 & 745.39 & 714.67 & & 702.45 & 748.27 & \\
\hline 7 & 739.12 & 703.72 & 747.66 & 712.18 & 735.56 & 700.14 & 750.68 & 715.20 \\
\hline 8 & 741.55 & 701.40 & 750.05 & 709.88 & 738.00 & 697.92 & 753.14 & 712.82 \\
\hline 9 & 743.99 & 699.09 & 752.33 & 707.52 & 740.57 & 695.60 & 755.65 & 710.45 \\
\hline 10 & 746.45 & 696.86 & 754.68 & 705.21 & 742.93 & 693.27 & 758.06 & \\
\hline 11 & 748.85 & 694.63 & 757.03 & 702.74 & & 691.03 & 760.56 & \\
\hline
\end{tabular}


TABLE 3 (Continued)

\begin{tabular}{|c|c|c|c|c|c|c|c|c|}
\hline \multirow[b]{2}{*}{$J$} & \multicolumn{2}{|c|}{$\begin{array}{c}2 v_{5}^{0}-v_{5} \\
\Sigma-\Pi\end{array}$} & \multicolumn{2}{|c|}{$\begin{array}{c}2 v_{5}^{2}-v_{5} \\
\Delta-\Pi\end{array}$} & \multicolumn{2}{|c|}{$\left(v_{4}+v_{5}\right)^{0}-v_{4}$} & \multicolumn{2}{|c|}{$\begin{array}{c}\left(v_{4}+v_{5}\right)^{2} \\
\Delta-\Pi\end{array}$} \\
\hline & $R(J)$ & $P(J)$ & $R(J)$ & $P(J)$ & $R(J)$ & $P(J)$ & $\mathrm{R}(\mathrm{J})$ & $P(J)$ \\
\hline 12 & 751.25 & 692.33 & 759.44 & 700.47 & & 688.78 & 763.30 & 703.72 \\
\hline 13 & 753.54 & 689.98 & 761.76 & 697.92 & & 686.59 & 765.55 & 701.52 \\
\hline 14 & 756.20 & 687.80 & 764.08 & 695.60 & & 684.38 & 768.10 & 699.29 \\
\hline 15 & 758.67 & 685.53 & 766.38 & 693.23 & & 682.18 & 770.65 & 697.06 \\
\hline 16 & 761.12 & 683.29 & 768.79 & 691.03 & 757.70 & 679.98 & & 694.89 \\
\hline 17 & 763.60 & 681.10 & 771.02 & 688.60 & 760.05 & 677.69 & & 692.70 \\
\hline 18 & 766.05 & 678.86 & 773.41 & 686.26 & 762.60 & 675.50 & & 690.39 \\
\hline 19 & 768.51 & 676.67 & 775.85 & 684.14 & 764.95 & 673.32 & & 688.19 \\
\hline 20 & 771.02 & 674.39 & & & 767.54 & 671.14 & & \\
\hline 21 & 773.42 & $672.19^{-}$ & & & 770.10 & 668.85 & & \\
\hline 22 & 775.85 & 669.94 & & & 772.59 & 666.70 & & \\
\hline 23 & & 667.62 & & & 775.14 & 664.55 & & \\
\hline
\end{tabular}


TABLE 3 (Continued)

\begin{tabular}{|c|c|c|c|c|c|c|c|c|}
\hline \multirow[b]{2}{*}{$\mathrm{J}$} & \multicolumn{2}{|c|}{$\begin{array}{c}2 v_{5}^{0}-v_{5} \\
\Sigma-\Pi\end{array}$} & \multicolumn{2}{|c|}{$\begin{array}{c}2 v_{5}^{2}-v_{5} \\
\Delta-\Pi\end{array}$} & \multicolumn{2}{|c|}{$\begin{array}{c}\left(v_{4}+v_{5}\right)^{0}-v_{4} \\
\Sigma-\Pi\end{array}$} & \multicolumn{2}{|c|}{$\begin{array}{c}\left(v_{4}+v_{5}\right)^{2}-v_{4} \\
\Delta-\Pi\end{array}$} \\
\hline & $R(J)$ & $P(J)$ & $R(J)$ & $P(J)$ & $R(J)$ & $P(J)$ & $R(J)$ & $P(J)$ \\
\hline 24 & & 665.40 & & & 777.66 & 662.27 & & \\
\hline 25 & & 663.17 & & & 780.26 & 660.19 & & \\
\hline 26 & & 660.96 & & & 782.75 & 657.97 & & \\
\hline 27 & 788.00 & 658.74 & & & 785.06 & 655.82 & & \\
\hline 28 & 790.51 & 656.51 & & & 787.56 & 653.60 & & \\
\hline 29 & 792.90 & 654.22 & & & & & . & \\
\hline 30 & 795.30 & 652.00 & & & & & & \\
\hline 31 & 797.72 & 649.87 & & & & & & \\
\hline 32 & 800.12 & 647.51 & & & & & & \\
\hline 33 & 802.46 & 645.28 & & & & & & , \\
\hline 34 & 804.86 & 643.00 & & & & & & \\
\hline 35 & 807.26 & & & & & & & \\
\hline
\end{tabular}


TABLE 4

SOME OF THE BAND CENTERS AND ROTATIONAL CONSTANTS OF ${ }^{12} \mathrm{C}_{2} \mathrm{H}_{2}\left(\mathrm{~cm}^{-1}\right)$

\begin{tabular}{|c|c|c|c|c|}
\hline $\begin{array}{l}\text { Assignment of } \\
\text { band }\end{array}$ & $\begin{array}{r}\left(v_{1} v_{2} v_{3} v_{4}^{l} v_{5}^{l}\right)^{\prime} \\
-\left(v_{1} v_{2} v_{3} v_{4}^{l} v_{5}^{l}\right)^{\prime \prime}\end{array}$ & $\begin{array}{l}\text { Species of } \\
\text { states }\end{array}$ & Band center ${ }^{a}$ & $B^{2}-B^{x}$ \\
\hline$v_{5}$ & $0000^{0} 1^{1} \leftarrow 0000^{0} 0^{0}$ & $\Pi_{\mathrm{u}} \leftarrow \Sigma_{\mathrm{g}}^{+}$ & 729.15 & $-0.00021_{4}^{b}$ \\
\hline $2 v_{5}^{0}-v_{5}$ & $0000^{0} 2^{0} \leftarrow 0000^{0} 1^{1}$ & $\Sigma_{g}^{+}+\Pi_{u}$ & 720.00 & $+0.004_{3}$ \\
\hline $2 v_{5}^{2}-v_{5}$ & $0000^{0} 2^{2} \leftarrow 0000^{0} 1^{1}$ & $\Delta_{\mathrm{g}} \leftarrow \Pi_{\mathrm{u}}$ & 728.86 & $-0.001_{3}$ \\
\hline$\left(v_{4}+v_{5}\right)^{0}-v_{4}$ & $0001^{1} 1^{1} \leftarrow 0001^{1} 0^{0}$ & $\Sigma_{u}^{+} \leftarrow \Pi_{g}$ & 716.43 & $+0.004_{8}$ \\
\hline$\left(v_{4}+v_{5}\right)^{2}-v_{4}$ & $0001^{1} 1^{1} \leftarrow 0001^{1} 0^{0}$ & $\Delta_{\mathrm{u}} \leftarrow \Pi_{\mathrm{g}}$ & $731.23^{c}$ & $+0.008_{5}$ \\
\hline
\end{tabular}

a Band centers are believed to be accurate to $\pm 0.02 \mathrm{~cm}^{-1}$. (For instance, the origin $\nu_{5}$ of ${ }^{12} \mathrm{C}^{13} \mathrm{CH}_{2}$ determined in the present work compares very well with the value derived in Reference 2.)

$\mathrm{b}$ Although the $\mathrm{P}$ and $\mathrm{R}$ structure of the $v_{5}$ band has been observed to $\mathrm{J}=45$, the $\left(D^{\prime}-D^{\prime \prime}\right)$ value has been evaluated to be hegligibly small within the accuracy of the present data. $c$ The $Q$ branch of this band was identified as belonging to the $2 v_{5}{ }^{2}-v_{5}$ band in
previous work (7). 
TABLE 5

VIBRATIONAL LEVELS OF ACETYLENE $\left(\mathrm{cm}^{-1}\right)$

\begin{tabular}{|c|c|c|c|}
\hline $\begin{array}{c}1 \\
\text { Energy Level }\end{array}$ & Observed & Obs.-Calc. I & Obs.-Calc. II ${ }^{\mathrm{b}}$ \\
\hline \multicolumn{4}{|l|}{ Present work: } \\
\hline $0000^{0} 1$ & 730.33 & 0.00 & +0.05 \\
\hline $0000^{0} 2^{0}$ & 1449.15 & 0.00 & -0.11 \\
\hline $0000^{0} 2^{2}$ & 1462.72 & 0.00 & -0.10 \\
\hline Data from previous work $(3,7)$ : & & & \\
\hline $0000^{0} 3^{1}$ & 2170.31 & +0.14 & +0.08 \\
\hline $0000^{0} 4^{0}$ & 2880.55 & +0.87 & +0.11 \\
\hline $0000^{0} 4^{2}$ & 2894.07 & +0.82 & +0.06 \\
\hline
\end{tabular}

${ }^{a}$ Employing the observed values for the three levels studied in the present work one obtains $\omega_{5}{ }^{0}=729.24, x_{55}=-2.33, g_{55}=3.42$. "Calculated $I^{\prime \prime}$ refers to values obtained by
using these constants.

${ }^{b}$ Combining all the six levels given in this table one obtains $\omega_{5}^{0}=729.15, x_{55}=-2.26$, $\mathrm{g}_{55}=3.39$, and "Calculated II" refers to the values derived by using these constants. For comparison, Plyler et al. (3) give $\omega_{5} 0=729.11, x_{55}=-2.27, g_{55}=3.45$. 
to the $\left(v_{4}+v_{5}\right)^{0}$ band of acetylene. Since that paper was written, a few revisions have been made in the formula for the combination relation $R(J-1)+P(J)$ as applied to bands like $\left(v_{4}+v_{5}\right)$ and $2 v_{5}$ for which the upper states are combinations of two perpendicular vibrations and are of species sigma. For such a case, Amat and Nielsen (10) have shown that

$$
\begin{aligned}
& R(J-1)+P(J)=2 v_{0}+2\left(B^{\prime}-B^{\prime \prime}\right) J^{2} \\
& -\delta_{0}\left\{\left[1+\frac{16\left(q_{i}^{0}+q_{i}^{0}\right)^{2}}{\Delta_{0}^{2}} J^{2}\left(J^{2}+1\right)\right]^{1 / 2}-1\right\}
\end{aligned}
$$

where $q_{i, j}^{0}=1 / 4$ of the $q_{i, j}$ used by Herzberg (1). For the 00011 leve1.

$$
\Delta_{0}=\left(v_{4}+v_{5}\right)^{2}-\left(v_{4}+v_{5}\right)^{0}=2\left(g_{45}-2 B^{*}\right)-r_{45},
$$

and for the 00002 level

$$
\delta_{0}=2 v_{5}^{2}-2 v_{5}^{0}=4\left(g_{55}-B^{2}\right)
$$

Using the values of the energy levels determined in the present chapter, it is possible to calculate $\Delta_{0}$ for the 00011 and 00002 levels from Eqs. (5) and (6). These values for $f_{0}$ can be compared with the ones derived from Eq. (4), using $q_{4}^{0}=1.30 \times 10^{-3} \mathrm{~cm}^{-1}$, $\mathrm{q}_{5}^{0}=1.15 \times 10^{-3} \mathrm{~cm}^{-1}$ given from theoretical considerations (11), the 
measurement of the $\left(v_{4}+v_{5}\right)^{0}$ band reported earlier, and the $2 v_{5}^{0}-v_{5}$ band measured in the present work. The results obtained are as follows: for the 00011 level, the value for $\phi_{0}$ derived from Eq. (5) is $14.7 \mathrm{~cm}^{-1}$, as compared to the value $12.7 \pm 1 \mathrm{~cm}^{-1}$ determined by employing Eq. (4); for the 00002 level the corresponding values are $8.8 \mathrm{~cm}^{-1}$ and $10.6 \pm 2 \mathrm{~cm}^{-1}$, respectively.

Vibrational Constants $\omega_{5}^{0}, x_{55}$, and $g_{55}$ of Acetylene:

The band centers given in Table 4 allow the calculation of some of the energy levels of this molecule, which in turn lead to the evaluation of the vibrational constants given in Eq. (1) in the preceding chapter. The results for the levels which involve the excitation of the $v_{5}$ vibration only have been given in Table 5. In this table "Observed" refers to vibrational band origins, which are related to the band centers of Table 4 in the following manner:

Vibrational band origin = Band center $+B^{\prime} l^{\prime 2}-B^{\prime \prime} l^{\prime \prime 2}$.

It is indeed true that inclusion of terms like $B l^{2}$ into the vibrational energy formula, as proposed in Herzberg's book (1) makes some matters simpler, however, the notation employed here were chosen to facilitate a comparison with the results published by Plyler $(3,12)$ and by $\operatorname{Keller}(7)$. The two sets of calculations given in Table 5 show that slight differences in vibrational constants can lead to large differences in the calculated values of the energy levels. Table 5 shows that the levels 
$0000^{0} 1^{1}, 0000^{0} 2^{0}, 0000^{0} 2^{2}, 0000^{0} 3^{1}, 0000^{0} 4^{0}$, and $0000^{0} 4^{2}$ can be fit to within a calculated-minus-observed disagreement of $0.1 \mathrm{~cm}^{-1}$. This is to be considered very satisfactory in comparison with the observed agreement or lack thereof for other vibrational levels of this molecule. The $0.1 \mathrm{~cm}^{-1}$ disagreement is only a bit larger than the uncertainty in some of the energy levels; however, it is not attributable to experimental error; it seems more likely to arise from the presence of the neglected cubic terms, which are apt to be of the order of magnitude of the discrepancies, that is, $0.1 \mathrm{~cm}^{-1}$. The measurement of the transition $\left(v_{4}+v_{5}\right)^{2}-v_{4}^{1}$ allowed us to determine the position of the level $0001^{ \pm 1} 1^{ \pm 1}$; this delta state had not been determined before, and the knowledge of its position proved invaluable in the calculation of the constants $x_{45}$ and $g_{45}$. Since this calculation is not as straightforward as that considered above, because of effect of vibrational l-type doubling, it is not considered here and is instead taken up in Chapter III.

$v_{5}$ Band of ${ }^{12} \mathrm{C}^{13} \mathrm{CH}_{2}$ :

As indicated in -Figs. $(1-3)$ and Table 2 , the $v_{5}$ fundamental vibration rotation band of the isotopically-substituted molecule ${ }^{12} \mathrm{C}^{13} \mathrm{CH}_{2}$ was identified and measured. Analysis of this band allowed us to locate its band center at $728.23 \mathrm{~cm}^{-1}$. This value agrees excellently with the value of $728.233 \mathrm{~cm}^{-1}$ quoted by Lafferty (2), who determined it indirectly from difference bands, using an isotopicallyenriched sample. In view of this agreement, and also because all the bands used in the present study have been observed in one spectral 
region, it is believed that a consistent set of vibrational constants have been obtained for the acetylene molecule.

Ground-state Rotationa1 Constants of ${ }^{12} \mathrm{C}_{2} \mathrm{H}_{2}$ :

The ground-state rotational constants of this molecule have been evaluated from the observational data for the fundamental. The results obtained are $B_{\text {ground state }}=1.1766_{6} \mathrm{~cm}^{-1} ; D_{\text {ground state }}=\left(1.8_{1} \pm 0.2\right)$ $x 10^{-6} \mathrm{~cm}^{-1}$. The accuracy with which these constants have been determined is limited by the blended nature of many of the rotational lines of the $v_{5}$ band, and the fact that the spectral positions in this work were obtained by employing a grating constant rather than the more modern methods. The present investigation has been done mainly with the intent of identifying the rotational lines of acetylene bands observed at 13.7 microns.

Identification of a Band Pertaining to ${ }^{12} \mathrm{C}_{2} \mathrm{H}_{2}$ at $2.5 \mu$ :

Combining the results obtained in the present study with the measurement reported for the $\left[\left(v_{1}+v_{4}+v_{5}\right)^{0}-v_{4}\right]$ band $(12)$, it has been possible to determine the level $\left(v_{1}+v_{4}+v_{5}\right)^{2}$. Based on this calculation, the $Q$ branch reported (12) at $4077.2 \mathrm{~cm}^{-1}$ has been assigned to the transition $\left[\left(v_{1}+v_{4}+v_{5}\right)^{2}-v_{4}\right]$.

\section{INTENSITY ALTERNATION IN ACETYLENE BANDS}

Although nothing unexpected or unusual has been noticed in the present data, it is believed that the spectrum shown in Figs. (1-3) provide a good illustration or the intensity alternation expected in 
the rotational structure of the vibration rotation bands of the linear symmetric $\mathrm{H}-{ }^{12} \mathrm{C}-{ }^{12} \mathrm{C}-\mathrm{H}$ molecule resulting from the $\operatorname{spin}(\mathrm{I}=1 / 2)$ of the $\mathrm{H}$ nucleus. It may be noted that in the bands $v_{5}\left(\Pi_{\mathrm{u}}-\Sigma_{\mathrm{g}}{ }^{+}\right)$ and $\left[\left(v_{4}+v_{5}\right)^{0}-v_{4}\right]\left(\Sigma_{\mathrm{u}}^{+}-\Pi_{\mathrm{g}}\right)$ lines with odd $\mathrm{J}$ are stronger, whereas in the band $2 v_{5}{ }^{0}-v_{5}\left(\Sigma_{g}^{+}-\Pi_{u}\right)$ the lines with even $J$ are the more intense. The $2 v_{5}{ }^{2}-v_{5}\left(\Delta_{g}-\Pi_{u}\right)$ and $\left[\left(v_{4}+v_{5}\right)^{2}-v_{4}\right]\left(\Delta_{u}-\Pi_{g}\right)$ bands show no intensity alternation. Also, as expected, the $v_{5}$ fundamental of ${ }^{12} \mathrm{C}^{13} \mathrm{CH}_{2}$ shows no intensity alternation. 


\section{CHAPTER III}

\section{VIBRATIONAL $\ell$-TYPE RESONANCE AND DOUBLING}

As indicated in Chapter $I$, there exist terms in the vibrational energy expression for acetylene which are diagonal in the total vibrational angular momentum about the figure axis but are not diagonal in the individual vibrational angular momenta associated with the transverse degenerate modes $v_{4}$ and $v_{5}$. Because of this, the portion of the Hamiltonian involving levels for which both transverse modes are excited is complicated by the presence of off-diagonal elements when the usual representation, labeling states by the quantum numbers $v_{i}$ and $l_{i}$, is made. The theoretical aspects of this effect are discussed in Reference 6 by Amat and Nielsen; here only their results are used, as given in Eq. (3) in Chapter I and repeated below:

$$
\begin{aligned}
& \left(\mathrm{v}_{4}, \ell_{4} ; \mathrm{v}_{5}, \ell_{5} ; \cdots\left|\mathrm{H}_{2}\right| \mathrm{v}_{4}, \ell_{4} \pm 2 ; \mathrm{v}_{5}, \ell_{5} \mp 2 ; \cdots\right) \\
& =1 / 4 \mathrm{hcr}_{45}\left[\left(\mathrm{v}_{4} \pm \ell_{4}+2\right)\left(\mathrm{v}_{4} \mp \ell_{4}\right)\left(\mathrm{v}_{5} \mp l_{5}+2\right)\left(\mathrm{v}_{5} \pm \ell_{5}\right)\right]^{1 / 2} .
\end{aligned}
$$

When terms of the form given in the equation above are included in the Hamiltonian and the resulting matrix is then diagonalized, the energy levels obtained are different from those derived ignoring such 
terms. When such a term occurs as the matrix element between two states which have different unperturbed energy levels $E_{1}$ and $E_{2}$ as their diagonal elements, the actual energy levels resulting from the presence of the perturbing off-diagonal element $R$ are $\left(E_{1}+E_{2}\right) / 2 \pm\left\{\left[1 / 2\left(E_{1}-E_{2}\right)\right]^{2}+R^{2}\right\}^{1 / 2}$, as can be easily verified by the diagonalization of the $2 \times 2$ matrix involved; this effect is termed "vibrational l-type resonance." When $\mathrm{E}_{1}=\mathrm{E}_{2}$, the energy levels which result are $E \pm R$ and the effect is termed "vibrational $b$-type doubling," where "doubling" signifies that the degeneracy of a level has been removed, creating two levels from one. Both the resonance and doubling effects are known in acetylene and are illustrated in Fig. 4. The cases shown are particularly simple in that no other resonances are involved and a maximum of two states are concerned; in the next chapter I shall deal with six states for which neither of these simplifications hold. Fig. 5 presents a somewhat complicated diagram showing the effects of both resonance and doubling within the 00031 states.

The energy level diagrams in Figs. 4 and 5 are most easily understood by reference to the matrices involved. These have been presented as Matrices I - VI. Matrices I, III, and V include both vibrational l-type terms and rotational l-type doubling terms (13); the rotational terms have been included because of the feasibility of inserting the numerical values for the $\mathrm{B}_{v}, \mathrm{D}_{\mathrm{v}}, \mathrm{q}_{i}$, and all of the vibrational constants, and then numerically diagonalizing these matrices for values of $\mathrm{J}$ up to, say, 20 by computer. Discrepancies between these calculated values and the observed rotational line positions of the vibrational levels observed in transitions would be an excellent measure of 
Matrix I

Vibration Rotation Submatrix Pertaining to the Levels 00011 


\begin{tabular}{|c|c|c|c|c|}
\hline & $0001^{+1} 1+1$ & $0001^{-1} 1^{1}$ & $0001^{+1} 1_{-1}$ & $0001^{-1} 1^{-1}$ \\
\hline $0001^{+1} 1+1$ & $\begin{array}{c}\omega_{4}{ }^{0}+w_{5}{ }^{0}+x_{44} \\
+x_{45}+X_{55} \\
+g_{44}+g_{45}+g_{55} \\
+F(J)\end{array}$ & $2 q_{4}^{0} J(J+1)$ & $2 q_{5}{ }^{0} J(J+1)$ & 0 \\
\hline $0001^{-1} 1+1$ & $2 q_{4}^{0} J(J+1)$ & $\begin{array}{c}\omega_{4}{ }^{0}+w_{5}{ }^{0}+X_{44} \\
+X_{45}+X_{55} \\
+g_{44}-g_{45}+g_{55} \\
+F(J)\end{array}$ & $r_{45}$ & $2 q_{5}^{0} J(J+1)$ \\
\hline $0001+{ }^{+} 1-1$ & $2 q_{5}^{0} J(J+1)$ & $r_{45}$ & $\begin{aligned} w_{4}{ }^{0} & +w_{5} o_{4} X_{44} \\
+ & X_{45}+X_{55} \\
+g_{44} & -g_{45}+g_{55} \\
& +F(J)\end{aligned}$ & $2 \mathrm{q}_{4}^{0} \mathrm{~J}(\mathrm{~J}+1)$ \\
\hline $0001^{-1} 1^{-1}$ & 0 & $2 q_{5}{ }^{0} J(J+1)$ & $2 q_{4}^{0} J(J+1)$ & $\begin{aligned} w_{4}{ }^{0} & +w_{5}{ }^{0}+X_{44} \\
& +x_{45}+X_{55} \\
+g_{44} & +g_{45}+g_{55} \\
& +F(J)\end{aligned}$ \\
\hline
\end{tabular}

MATRIX I 
Matrix II

Vibration Submatrix Pertaining to 00011 


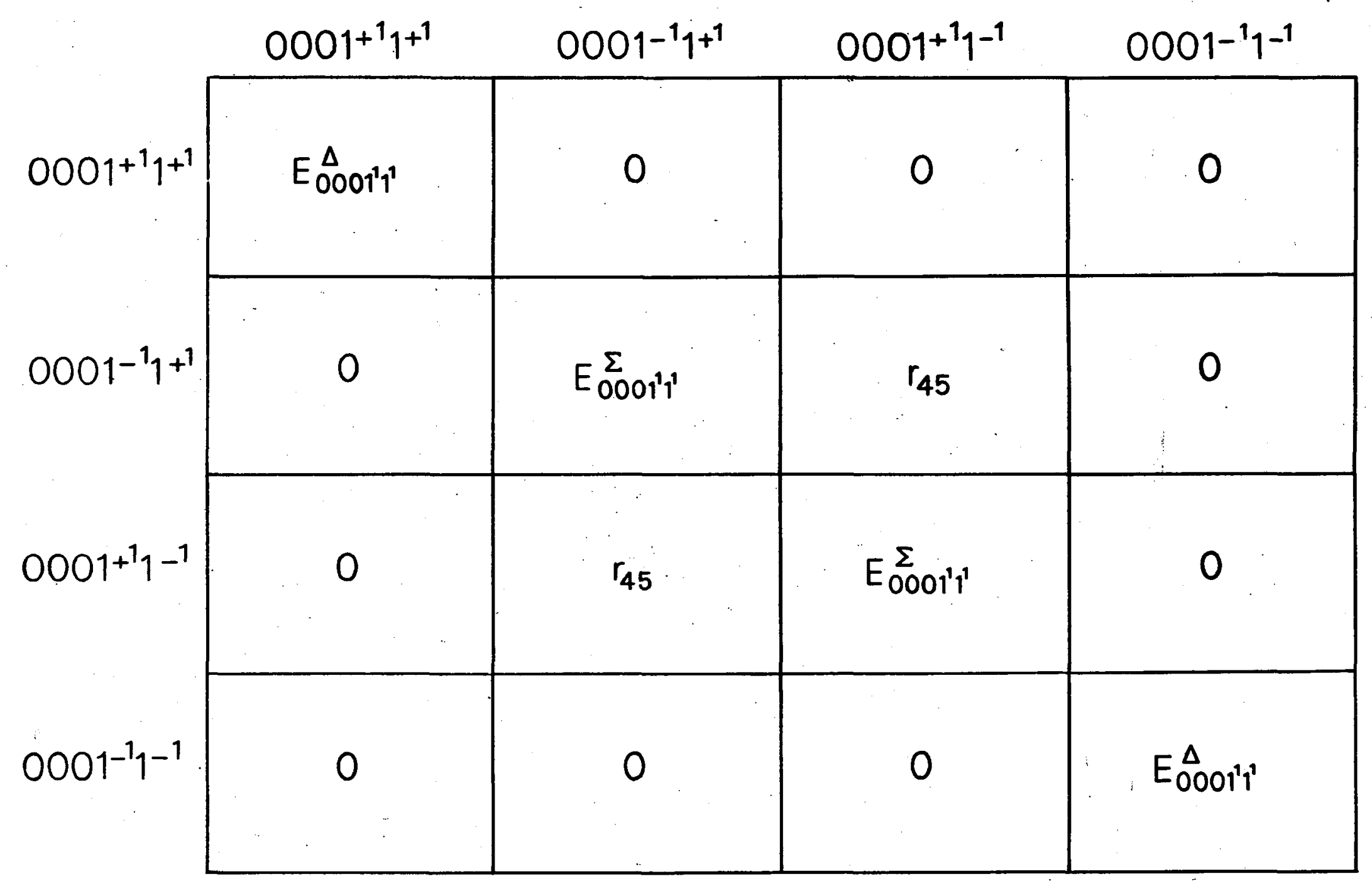

MATRIX II 


\section{Matrix III}

Vibration Rotation Submatrix Pertaining to the Levels 00021 


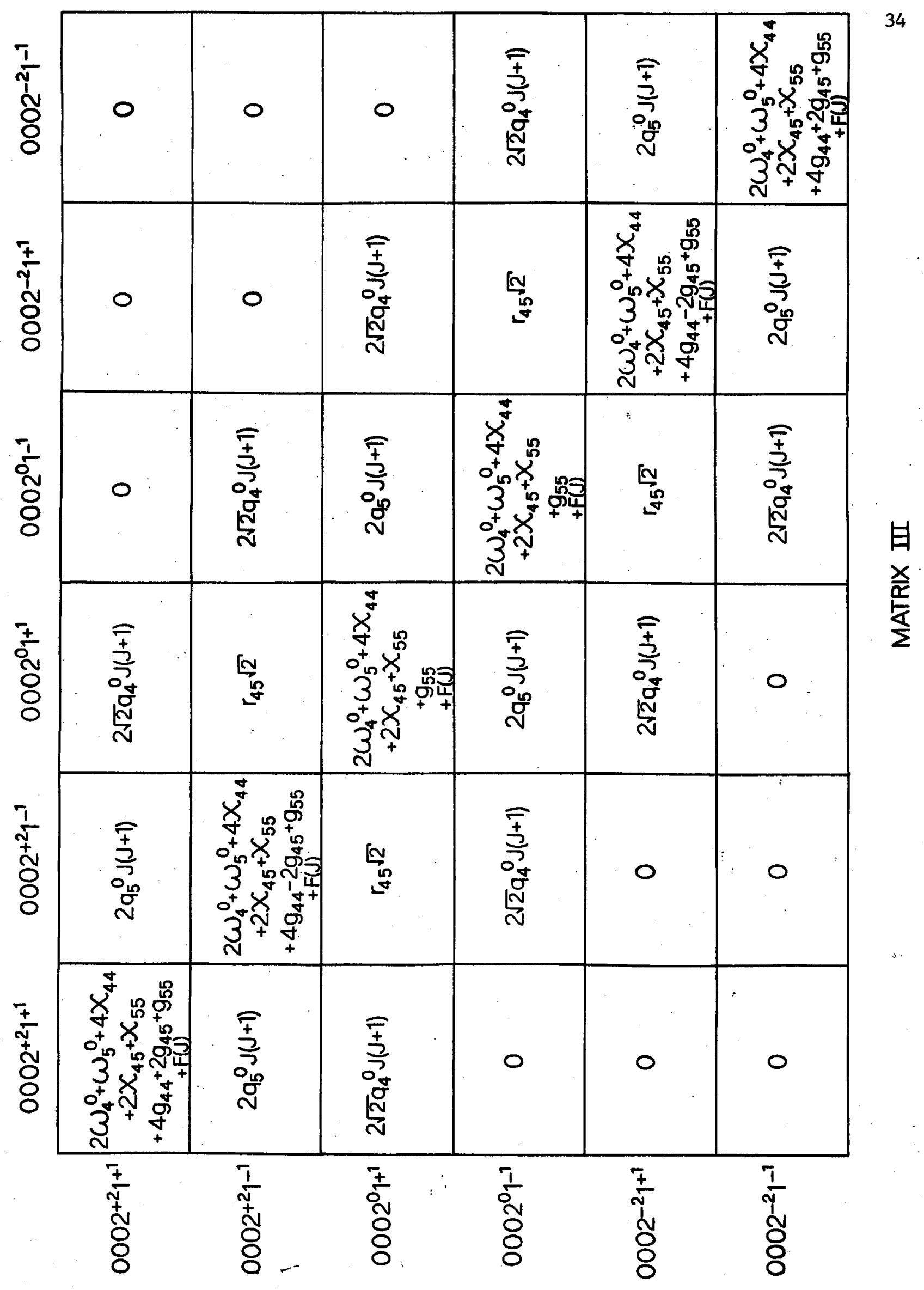


Matrix IV

Vibration Submatrix Pertaining to 00021 


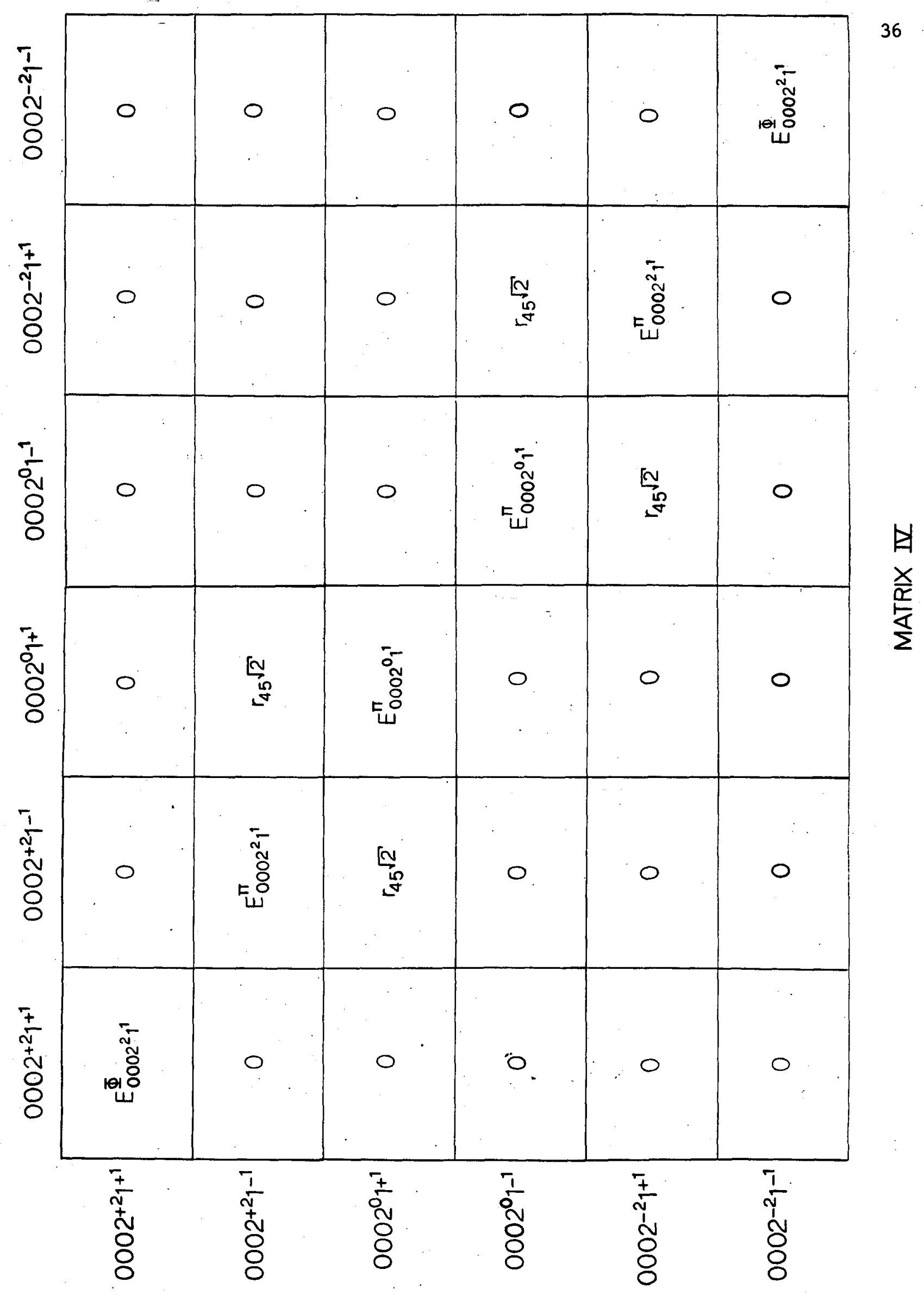


Matrix V

Vibration Rotation Submatrix Pertaining

to the Levels 00031 


\begin{tabular}{|c|c|c|c|c|c|c|c|c|}
\hline & $0003^{+3} 1^{1}$ & $0003^{+3} 1_{1}^{-1}$ & $0003^{+1} 1+1$ & $0003^{+1} 1^{-1}$ & $0003^{-1} 1^{1}$ & $0003^{-1} 1^{-1}$ & $0003^{-3_{1}+1}$ & $\left.0003^{-3}\right\}_{1}^{-1}$ \\
\hline $0003^{3}{ }^{3} 1^{1}$ & $\begin{array}{c}3 \omega_{4}^{0}+\omega_{5}^{0}+9 x_{44} \\
+3 x_{45}+x_{55} \\
+9 g_{44}+3 g_{45}+g_{55} \\
+F(N)\end{array}$ & $2 q_{5}^{0} \mathrm{~J}(\mathrm{~J}+1)$ & $\begin{array}{l}2 \sqrt{3} a_{4}^{0}[[J(J+1) \\
-2][J(J+1)-6]\}^{1 / 2}\end{array}$ & 0 & 0 & 0 & 0 & 0 \\
\hline $0003^{3} 1^{-3}$ & $2 q_{5}^{0} \mathrm{~J}(\mathrm{~J}+1)$ & $\mid \begin{array}{c}3 \omega_{4}^{0}+\omega_{5}^{0}+9 x_{44} \\
+3 x_{45}+x_{55} \\
+9 g_{44}-3 g_{45}+g_{55} \\
+F(v)\end{array}$ & $\sqrt{3} r_{45}$ & $\begin{array}{l}2 \sqrt{3} q_{4}^{0}\{[J(J+1) \\
-2][J(J+1)-6]]^{1 / 2}\end{array}$ & 0 & 0 & 0 & 0 \\
\hline $.0003^{+1} 1^{+1}$ & $\begin{array}{l}2 \sqrt{3} q_{4}^{0}[[\mathrm{~J}(\mathrm{~J}+1) \\
-2][\mathrm{J}(\mathrm{J}+1)-6]]^{1 / 2}\end{array}$ & $\sqrt{3} r_{45}$ & $\begin{array}{l}3 \omega_{4}^{0}+\omega_{5}^{0}+9 X_{44} \\
+3 X_{45}+X_{55} \\
+g_{44}+g_{45}+g_{55} \\
+F(J)\end{array}$ & $2 q_{5}^{0} J(J+1)$ & $4 q_{4}^{0} J(J+1)$ & 0 & 0 & 0 \\
\hline $0003^{+1} q^{-1}$ & 0 & $\begin{array}{l}2 \sqrt{3} q_{4}^{0}\{[[(J+1) \\
-2][J(J+1)-6]]^{1 / 2}\end{array}$ & $2 q_{5}^{0} J(J+1)$ & $\left\{\begin{array}{c}3 \omega_{4}^{0}+\omega_{5}^{0}+9 x_{44} \\
+3 x_{45}+x_{55} \\
+g_{44}-g_{45}+g_{55} \\
+F(j)\end{array}\right.$ & $2 r_{45}$ & $4 \mathrm{q}_{4}^{0} \mathrm{~J}(\mathrm{~J}+1)$ & 0 & 0 \\
\hline $0003^{-1} 1^{+1}$ & 0 & 0 & $4 q_{4}^{0} J(J+1)$ & $2 r_{45}$ & $\begin{array}{c}3 \omega_{4}^{0}+\omega_{5}^{0}+9 x_{44} \\
+3 x_{45}+x_{55} \\
+g_{44}-g_{45}+g_{55} \\
+F(J)\end{array}$ & $2 q_{5}^{0} J(J+1)$ & $\begin{array}{l}2 \sqrt{3} q_{4}^{0}\{[J(J+1) \\
-2][J(J+1)-6]\}^{1 / 2}\end{array}$ & 0 \\
\hline $0003^{-1} 1^{-1}$ & 0 & 0 & 0 & $4 q_{4}^{0} J(J+1)$ & $2 q_{5}^{0} \mathrm{~J}(J+1)$ & $\begin{array}{c}3 \omega_{4}^{0}+\omega_{5}^{0}+9 x_{44} \\
+3 x_{45}+x_{55} \\
+g_{44}+g_{45}+g_{55} \\
+F(j)\end{array}$ & $\sqrt{3} r_{45}$ & $\begin{array}{l}2 \sqrt{3} \mathrm{q}_{4}^{0}[[J(J+1) \\
-2][(J+1)-6]]^{1 / 2}\end{array}$ \\
\hline $0003^{-3} 1^{+1}$ & 0 & 0 & 0 & 0 & $\begin{array}{l}2 \sqrt{3} q_{4}^{0}[[\mathrm{~J}(\mathrm{~J}+1) \\
-2][\mathrm{J}(\mathrm{J}+1)-6]]^{1 / 2}\end{array}$ & $\sqrt{3} r_{45}$ & $\mid \begin{array}{c}3 \omega_{4}^{0}+\omega_{5}^{0}+9 x_{44} \\
+3 x_{45}+x_{55} \\
+9 g_{44}-3 g_{45}+g_{55} \\
+F(J)\end{array}$ & $2 q_{5}^{0} \mathrm{~J}(\mathrm{~J}+1)$ \\
\hline $0003^{-3} 1^{-1}$ & 0 & 0 & 0 & 0 & 0 & $\begin{array}{l}2 \sqrt{3} \mathrm{q}_{4}^{0}\{[\mathrm{~J}(\mathrm{~J}+1) \\
-2][\mathrm{J}(\mathrm{J}+1)-6]]^{1 / 2}\end{array}$ & $2 q_{5}^{0} \mathrm{~J}(\mathrm{~J}+1)$ & $\begin{array}{c}3 \omega_{4}^{0}+\omega_{5}^{0}+9 x_{44} \\
+3 x_{45}+x_{55} \\
+9 g_{44}+3 g_{45}+g_{55} \\
+F(J)\end{array}$ \\
\hline
\end{tabular}

MATRIX $\mathbb{Z}$ 
Matrix VI

Vibration Submatrix Pertaining to 00031 


\begin{tabular}{|c|c|c|c|c|c|c|c|c|}
\hline & $0003^{3} 3^{3}+1$ & $0003^{+3} 1^{-1}$ & $0003^{+1} 1^{+1}$ & $0003^{+1} 1^{-1}$ & $0003^{-1} 1^{1}$ & $0003^{-1} 1^{-1}$ & $0003^{-3} 3^{3}+1$ & $0003^{-3_{1}-1}$ \\
\hline $0003^{3}{ }^{3} 1+1$ & $E_{0003^{3}}^{r}$ & 0 & 0 & 0 & 0 & 0 & 0 & 0 \\
\hline $0003^{3} 1^{3}-1$ & 0 & $E_{0003_{1}^{3}}^{\Delta}$ & $\sqrt{3} r_{45}$ & 0 & 0 & 0 & 0 & 0 \\
\hline $0003^{+1} 1^{+1}$ & 0 & $\sqrt{3} r_{45}$ & $E_{0003^{\prime} 1^{\prime}}^{\Delta}$ & 0 & 0 & 0 & 0. & 0 \\
\hline $0003^{+1} 1^{-1}$ & 0 & 0 & 0 & $E_{0003^{\prime} 1^{1}}^{\Sigma}$ & $2 r_{45}$ & 0 & 0 & 0 \\
\hline $0003^{-1} 1^{+9}$ & 0 & 0 & 0 & $2 r_{45}$ & $E_{0003^{1} 1^{\prime}}^{\Sigma}$ & 0 & 0 & 0 \\
\hline $0003^{-1} 1^{-1}$ & 0 & 0 & 0 & 0 & 0 & $E_{0003^{\prime} \prime^{\prime}}$ & $\sqrt{3}_{r_{45}}$ & 0 \\
\hline $0003^{-3} 1^{3}+1$ & 0 & 0 & 0 & 0 & 0 & $\sqrt{3} r_{45}$ & $E_{0003^{3} y^{1}}^{\Delta}$ & 0 \\
\hline $0003^{-3} 1^{-1}$ & 0 & 0 & 0 & 0 & 0 & 0 & 0 & $E_{0003^{3} 1^{1}}^{r}$ \\
\hline
\end{tabular}

MATRIX ZI 
Figure 4

The Energy Levels 00011 and 00021 

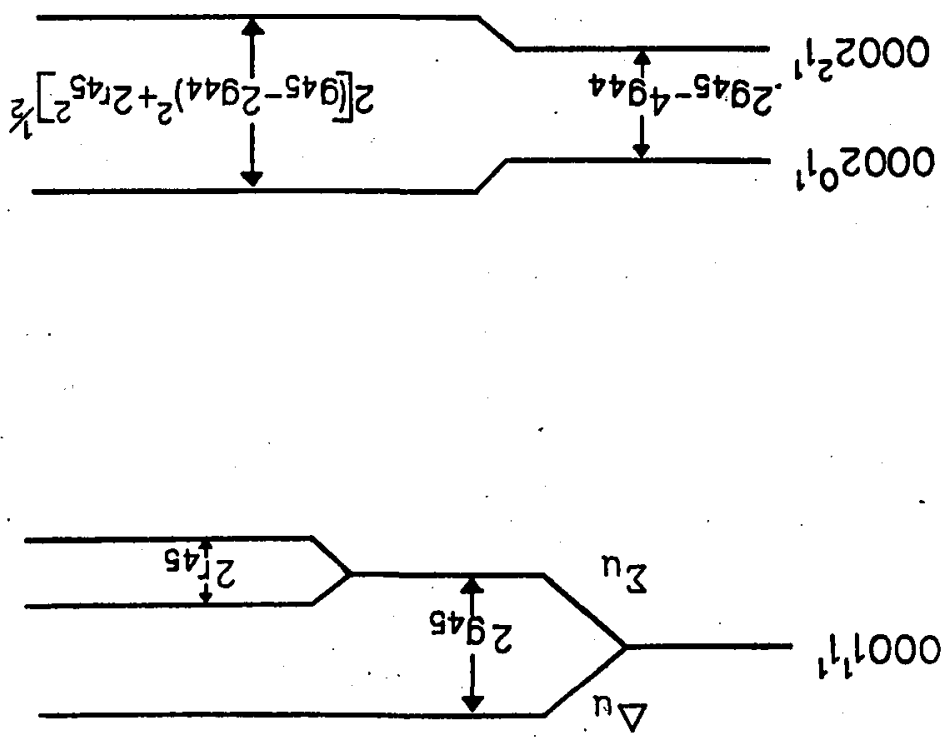

27 
Figure 5

The Energy Leve1s 00031 


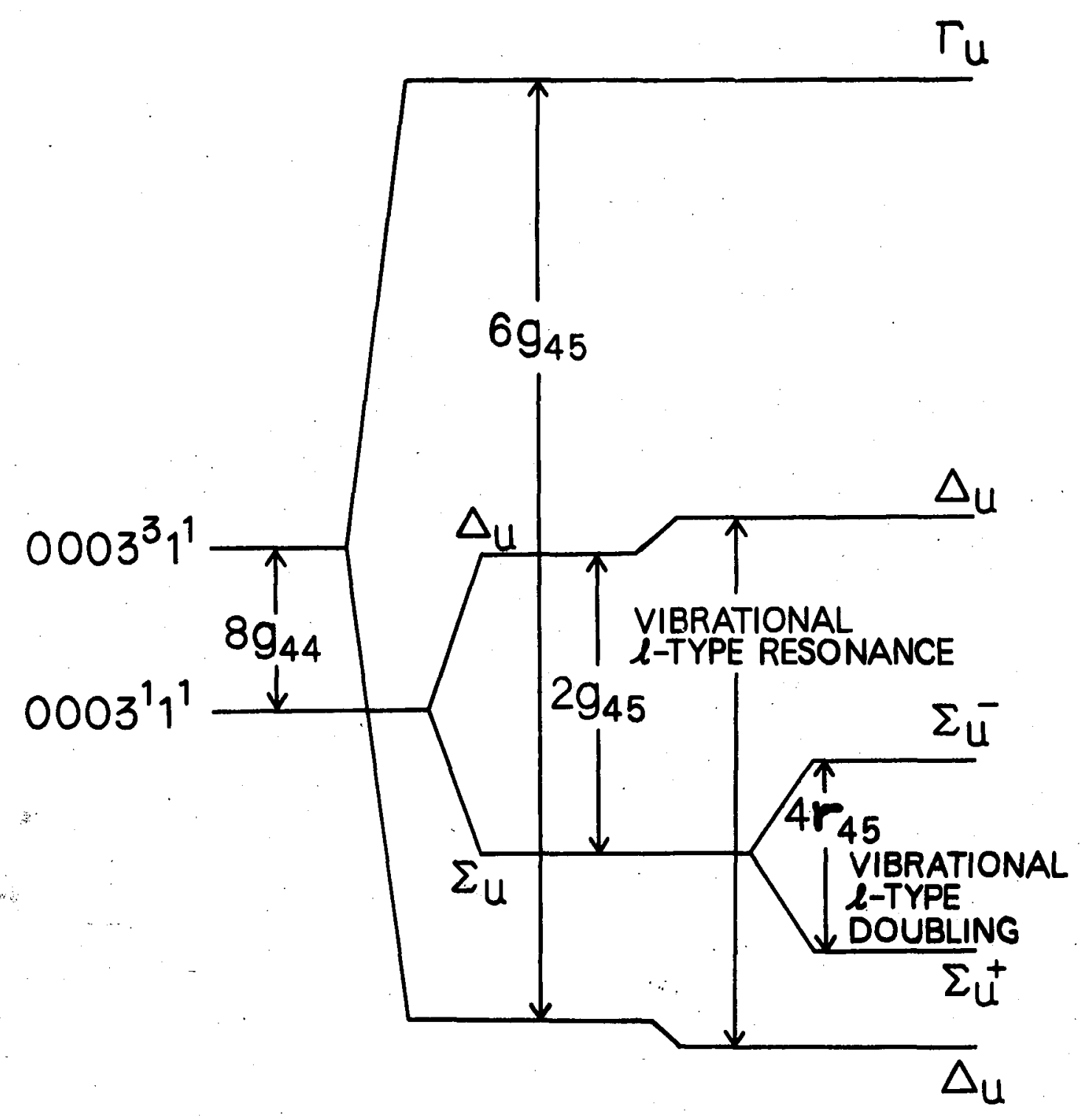


the uncertainty in the constants involved. This has not been done in the present work, partly because it would require the use of rotational constants obtained almost entirely from the works of other investigators, and primarily because the vibrational problem has been given greater emphasis. Matrices II, IV, and VI are vibrational submatrices of the Hamiltonian and do not include rotational terms. These three matrices, as is immediately evident, factor to a collection of $1 \mathrm{X} 1$ and $2 \mathrm{X} 2 \mathrm{sub}$-. matrices; hence, for each, the diagonalization is trivial. For the levels 00011 there is one 2X2; its diagonalization produces the doubling shown in Fig. 4. For the levels 00021, the pi-states involve a 2X2 which gives rise to the resonance. shown in Fig. 4. The leve1s 00031 involve $2 \times 2$ submatrices both for the case in which $\ell_{4}+l_{5}=0$ and where $l_{4}+l_{5}= \pm 2$; this gives rise to doubling of the sigmastate and resonance between the delta-states, as shown diagrammatically in Fig. 5.

By the diagonalization of these three matrices, algebraic relations for the actual perturbed vibrational energy levels have been obtained. The positions of these energy levels determined from the measurement of observed transitions have then been used to calculate the constants, diagonal and nondiagonal, involved.

Upon diagonalizing Matrix II we obtain a modification of Eq. (1):

$$
G_{0}(v)=w_{4}^{0}+w_{5}^{0}+x_{44}+x_{45}+x_{55}+g_{44}-g_{45}+g_{55} \pm r_{45} \text {, }
$$

where the plus sign refers to the $\Sigma^{+}$state and the minus sign to the $\Sigma^{-}$state; and for the $\Delta$ state, 


$$
G_{0}(v)=w_{4}^{0}+w_{5}^{0}+x_{44}+x_{45}+x_{55}+g_{44}+g_{45}+g_{55} \text {. }
$$

Chapter I discusses the measurement of both the $\left(v_{4}+v_{5}\right)^{0}-v_{4}$ and $\left(v_{4}+v_{5}\right)^{2}-v_{4}$ bands, and from the data given there one can arrive at the following two relations, using Eqs. (8-9), (units are $\mathrm{cm}^{-1}$ ):

$$
\begin{gathered}
{\left[\left(v_{4}+v_{5}\right)^{0}-v_{4}\right]-v_{5}=\mathrm{x}_{45}-\mathrm{g}_{45}+\mathrm{r}_{45}=-15.03,} \\
{\left[\left(v_{4}+v_{5}\right)^{2}-v_{4}\right]-v_{5}=\mathrm{x}_{45}+\mathrm{g}_{45}=+4.47 .}
\end{gathered}
$$

Another pair of useful levels for evaluating the constants $x_{45}, g_{45}$, and $r_{45}$ are the states $0002^{0} 1^{1}$ and $0002^{2} 1^{1}$. For these levels, the work of Amat and Nielsen (6) leads to the following expression ${ }^{a}$ for the vibrational term values:

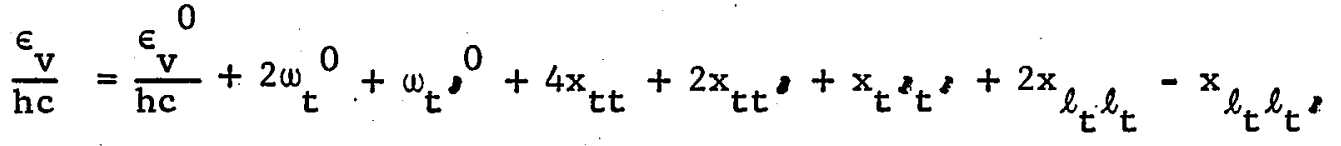

$$
\begin{aligned}
& +x_{l_{t} l_{t}} \pm\left\{\left(-2 x_{\ell_{t} \ell_{t}}+x_{l_{t} \ell_{t}}\right)^{2}+2 r_{t t^{\prime}}\right\}^{1 / 2}
\end{aligned}
$$

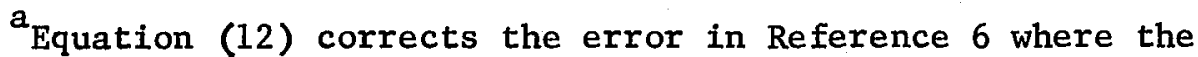
$r_{t t}$ term was raised to only a single power. 
In the notation of the present paper, Eq. (12) becomes

$$
\begin{aligned}
G_{0}(v)=2 w_{4}^{0}+w_{5}^{0}+4 x_{44} & +2 x_{45}+x_{55}+2 g_{44}-g_{45}+g_{55} \\
& \pm\left\{\left(-2 g_{44}+g_{45}\right)^{2}+2 r_{45}^{2}\right\}^{1 / 2},
\end{aligned}
$$

where the plus sign is taken for the $0002{ }^{0} 1^{1}$ level, and the minus sign. for $0002^{2} 1^{1}$. The above is, of course, simply the result obtained by the diagonalization of Matrix IV. Inserting into Eq. (13) the experimental data of Plyler et al., for the $2 v_{4}{ }^{0,2}+v_{5}$ bands ${ }^{b}$ and the value for the level $0002^{2} 0^{0}$ obtained from the work of Innes (14), it is deduced that

$$
\begin{aligned}
& {\left[\left(2 v_{4}^{0}+v_{5}\right)\right]-\left(2 v_{4}^{2}\right)-\left(v_{5}\right)=2 x_{45}-2 g_{44}-g_{45}} \\
& +\left\{\left(g_{45}-2 g_{44}\right)^{2}+2 r_{45}^{2}\right\}^{1 / 2}=-4.52, \\
& {\left[\left(2 v_{4}^{2}+v_{5}\right)\right]-\left(2 v_{4}^{2}\right)-\left(v_{5}\right)=2 x_{45}-2 g_{44}-g_{45}} \\
& -\left\{\left(g_{45}-2 g_{44}\right)^{2}+2 x_{45}^{2}\right\}^{1 / 2}=-24.23 .
\end{aligned}
$$

$\mathrm{b}_{\text {The }} \pm$ signs in Eq. (12) are associated with the wrong levels in Reference 6; this may be verified by setting $r_{t+}=0$ in Eq. (12) and noting that the expressions reduce to the correct form for the unperturbed energy levels given by the \pm assignments following Eq. (13). With this consideration, Eq. (12) indicates that the assignment of these bands given by Plyler et al. must be reversed, and we have done so in Eqs. (14) and (15). 
It is of interest to mention that the investigations by Innes deal with the electronic bands of the acetylene molecule. Figure 6 presents an energy level diagram wherein the levels of interest to the present paper are drawn. It is easy to see that the electronic transitions marked (i) and (ii) in the figure originate from the same upper electronic level and terminate in the levels $0002^{2} 0^{0}$ and the ground state $0000^{\circ} 0^{0}$. The simultaneous solution of Eqs. (10), (11), (13), (14) and (15) yields the roots given below in Table 6:

\section{TABLE 6}

\section{Set A}

$g_{45}$

$x_{45}$

$r_{45}$

$g_{44}$

$$
7.92
$$$$
-3.45
$$$$
-3.66
$$

$-0.22$
Set B

$$
6.35
$$

$-1.88$

$-6.80$

It was hoped that a choice between the two sets of roots given above could be made on the basis of an independent determination of the constant $\mathrm{g}_{44^{\circ}}$. Such a determination is afforded by the observational data of Innes (14) on the levels $0002^{2} 0^{0}, 0003^{1} 0^{0}$, and $0004^{0} 0^{0}$ and the level $0001^{1} 0^{0}$ known (1) from work in the infrared: The value obtained from Innes levels, however, is $g_{44}=1.36 \mathrm{~cm}^{-1}$ and is about equally far from each of the roots in Table 6, offering no help in the choice between them. The reason that Innes' value does not agree better with the results given here is probably as follows: the levels $0001^{1} 0^{0}$, $0002^{2} 0^{0}, 0003^{1} 0^{0}, 0004^{0} 0^{0}$ appear to yield a set of four equations in 
Figure 6

$$
\text { An Energy Level Diagram of }{ }^{12} \mathrm{C}_{2} \mathrm{H}_{2}
$$




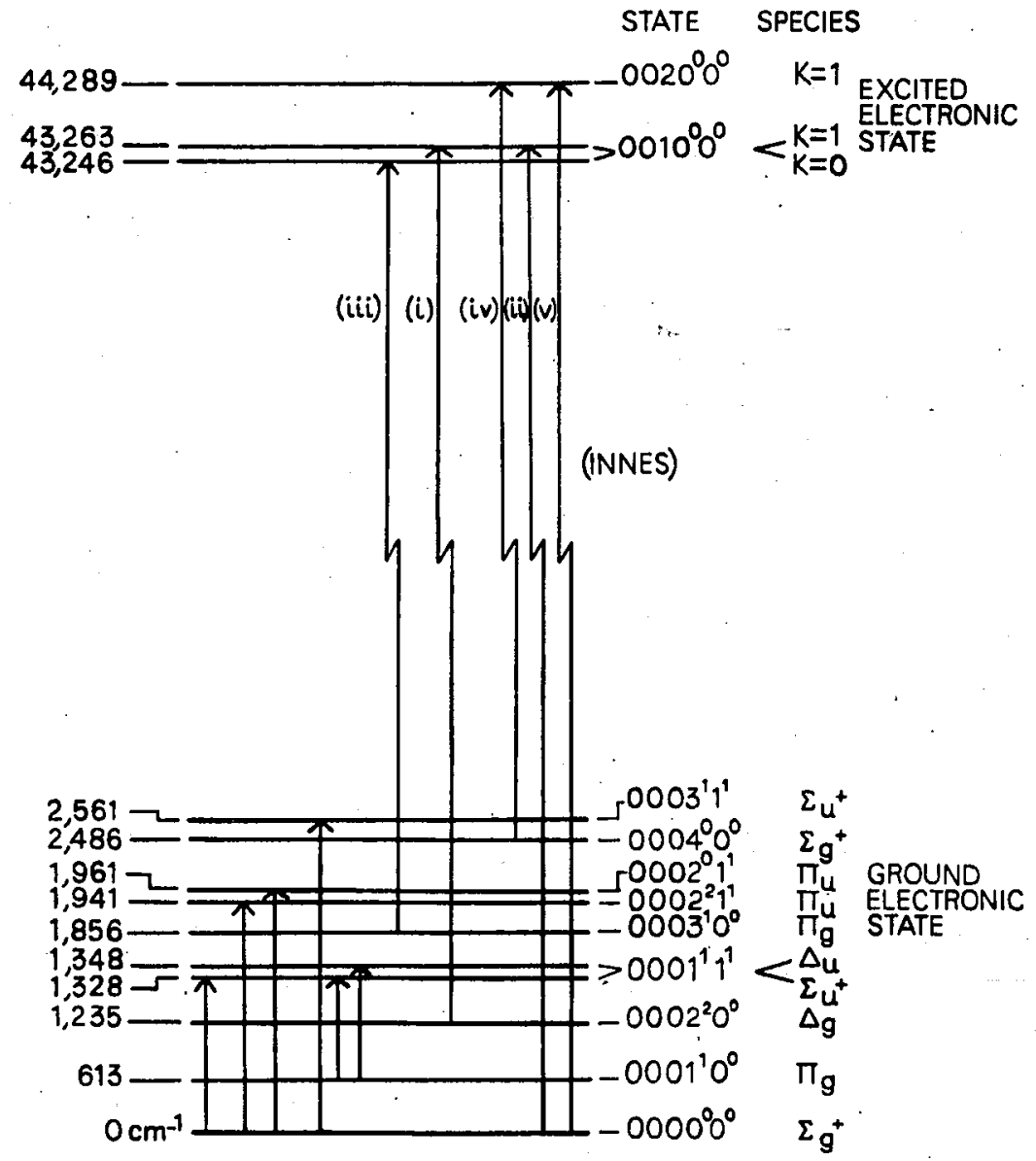


the three unknowns $w_{4}^{0}, x_{44}$, and $g_{44}$, but actually one of the equations involved is linearly dependent upon the others; hence, while all three constants can be calculated, and the fourth equation shows the selfconsistency of the experimental data, there is no real check, algebraically speaking, on the value of $\mathrm{g}_{44}$; moreover, since the levels involved do not include a pair such as $0002^{0} 0^{0}$ and $0002^{2} 0^{0}$ or $0003^{1} 0^{0}$ and $0003^{3} 0^{0}$, from which $g_{44}$ can be determined directly, this calculation is, in fact, rather insensitive to the value of $g_{44}$, which is very much smaller than that of $\omega_{4}^{0}$. Therefore the disagreement of both of our values of $g_{44}$ with that obtainable from Innes' work is not too disturbing.

An additional means of distinguishing between the true and extraneous roots in Table 6 was to be found, it had been hoped, in the levels 00031 presented in Fig. 5 and Matrix VI. The sigma-plus level of $0003^{1} 1^{1}$ has been observed by others (3) and yields the following algebraic expression upon diagonalization of Matrix VI:

$$
\begin{array}{r}
G_{0}(v)=3 w_{4}{ }^{0}+w_{5}{ }^{0}+9 x_{44}+3 x_{45}+x_{55}+g_{44} \\
-g_{45}+g_{55}+2 r_{45}
\end{array}
$$

This leads to the relation (using constants in Table 6)

$$
\left[\left(3 v_{4}+v_{5}\right)^{0}\right]-3 v_{4}-v_{5}=3 \mathrm{x}_{45}-\mathrm{g}_{45}+2 \mathrm{r}_{45}=-25.59
$$


Inserting the observational data for the infrared bands $\left(3 v_{4}+v_{5}\right)^{0}$ and $v_{5}$ along with the value for the level $0003^{1} 0^{0}$ determined from the studies of the electronic bands, the left-hand side of Eq. (17) is evaluated as -25.50 which compares excellent1y with the value of -25.59. based on the constants $\mathrm{x}_{45}, \mathrm{~g}_{45}$, and $\mathrm{r}_{45}$. Unfortunately, this expression is linearly dependent upon the expressions in Eqs. (10-11) and therefore offers no way of choosing between the two sets of roots in Table 6; it is satisfied equally well by each. It does serve to demonstrate the self-consistency of our equations, however, illustrating that the theoretically-derived equations are completely compatible with the data. Many other levels have been reported for acetylene involving the vibrational modes $v_{4}$ and $v_{5} \cdot \mathrm{All}$ these have one of the combinations $v_{4}+v_{5}, 2 v_{4}+v_{5}$, or $3 v_{4}+v_{5}$ occurring in them and therefore do not provide us with any more information than that discussed above insofar as the effect of $r_{45}$ term is concerned.

The next chapter shows, however, that the constants in Set $A$ of Table 6 are much more likely to approximate the true values than are those in Set B. This conclusion is drawn from the consideration of several levels involved as the upper states of difference bands in the three micron region. Since the calculations involve resonating triads which exhibit combined Fermi and vibrational l-type resonance, the effect is not simply that discussed in this chapter, and the details are presented in the next chapter, following the analysis of the bands involved. 


\section{CHAPTER IV}

\section{ANALYSIS OF THE BANDS AT THREE MICRONS}

Acetylene bands occurring in the three micron region of the spectrum have been studied by a number of different investigators $(1,2,3,6)$. In this region, one observes the bands $v_{3}, v_{2}+v_{4}+v_{5}$ and several overlapping "hot" bands (see Fig. 7), one of which was previously assigned (3) as $v_{2}+2 v_{4}^{0}+v_{5}-v_{4}$. There are seven "hot" bands which are expected to be of detectable intensity at normal temperatures; they are given in Fig. 8. Since all are of the $\Pi$ - $\Pi$ variety and are expected to exhibit resolvable rotational l-type doubling, the spectrum is very complex. In the present work, it has been possible to analyze six bands of ${ }^{12} \mathrm{C}_{2} \mathrm{H}_{2}$, including four "hot" bands, and two bands of ${ }^{12} \mathrm{C}^{13} \mathrm{CH}_{2}$; the carbon-13 transitions were comparable in intensity to the ${ }^{12} \mathrm{C}_{2} \mathrm{H}_{2}$ "hot" bands, although an unenriched sample of acetylene was used. Fig. 7 shows a fast scan of the spectrum in the region around three microns, with the rotational assignments given for $v_{3}$ and $v_{2}+v_{4}+v_{5}$. Slow scans with complete assignment of overlapping rotational structure are given in Appendix I.

Table 7 is a complete list of transitions observed at three microns. 
Figure 7

The Spectrum at 3 Microns 


$$
\mathrm{C}_{2} \mathrm{H}_{2} \text { at } 3 \mu
$$
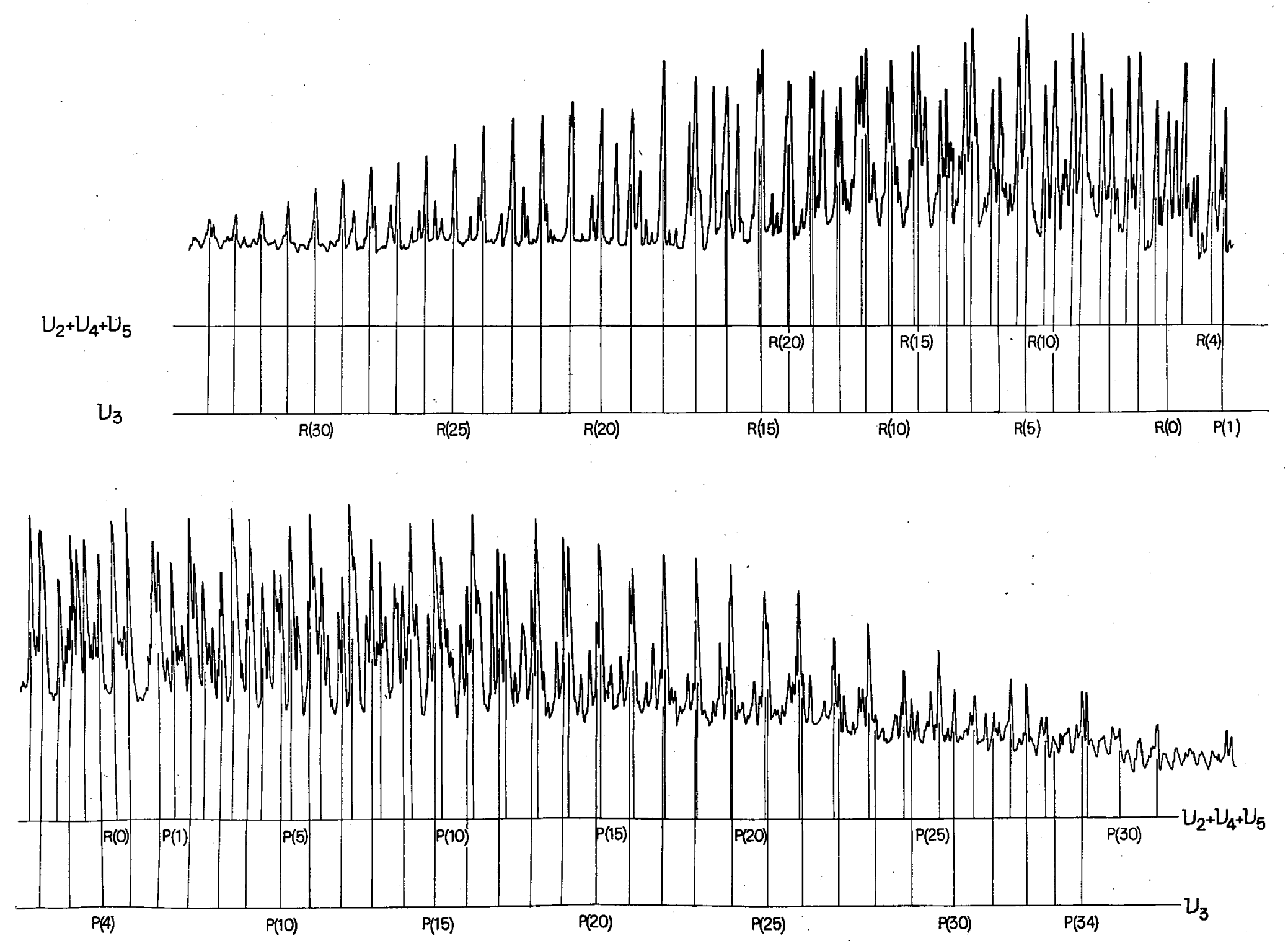


\section{Figure 8}

Transitions Expected at 3 Microns 

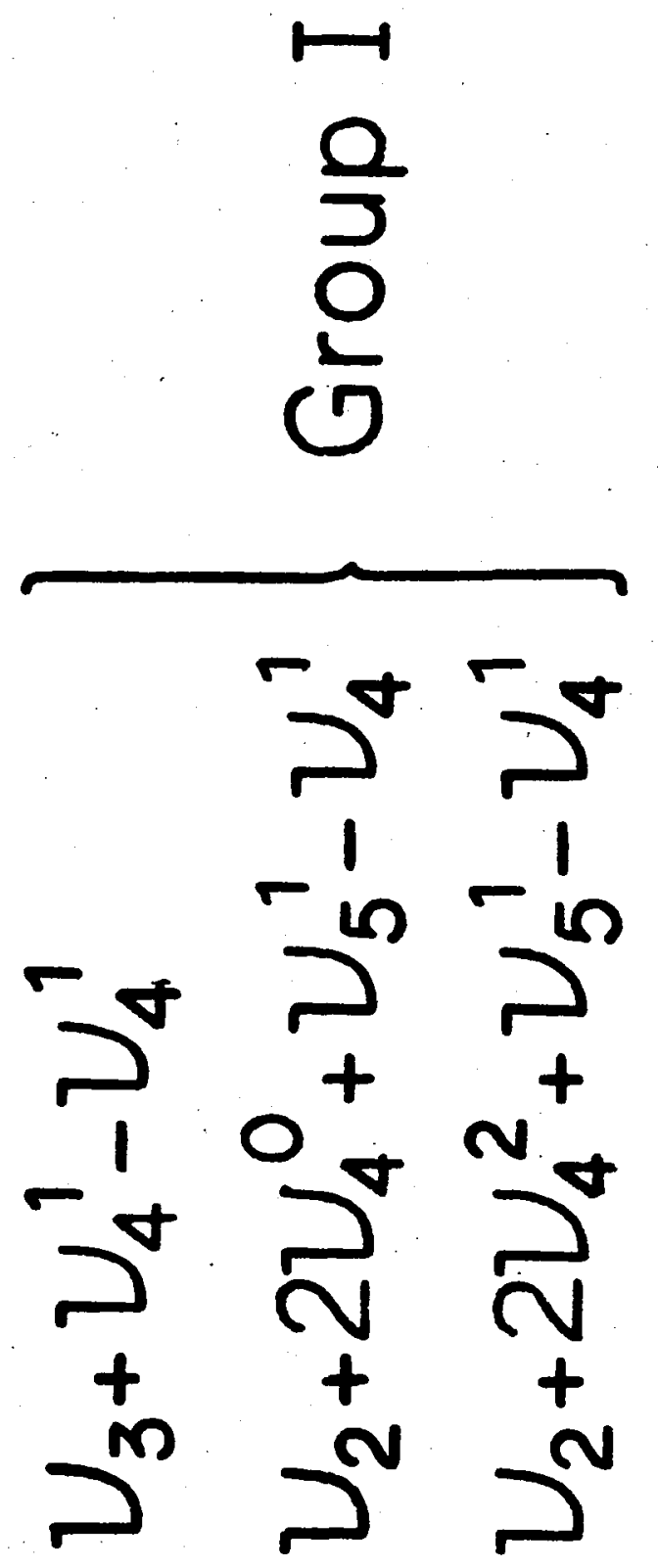

ロ

응

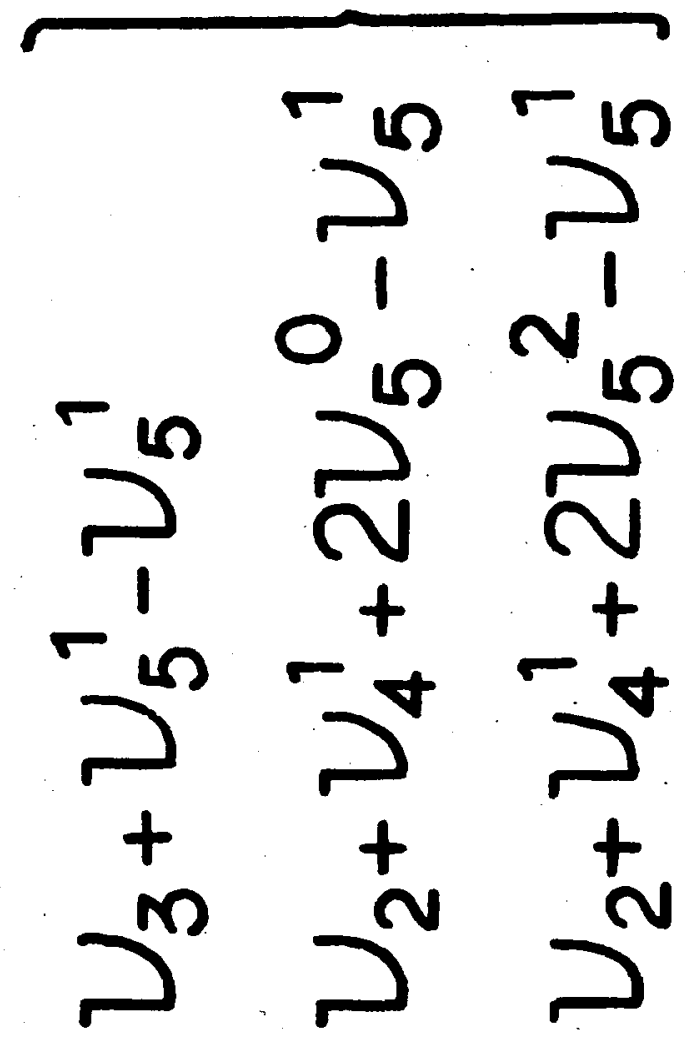


TABLE 7

TRANSITIONS OBSERVED AT THREE MICRONS

\begin{tabular}{lcc}
\hline UPPER STATE & LOWER STATE & BAND CENTER $\left(\mathrm{cm}^{-1}\right)$ \\
\hline $0010^{0} 0^{0}$ & $0000^{0} 0^{0}$ & 3294.822 \\
$0101^{1} 1^{1}$ & $0000^{0} 0^{0}$ & 3281.894 \\
$0011^{1} 0^{0}$ & $0001^{1} 0^{0}$ & 3285.44 \\
$0010^{0} 1^{1}$ & $0000^{0} 1^{1}$ & 3269.55 \\
$0102^{2} 1^{1}$ & $0001^{1} 0^{0}$ & 3269.70 \\
$0101^{1} 2^{2}$ & $0000^{0} 1^{1}$ & 3286.39 \\
\hline
\end{tabular}


TRANSITIONS FROM THE GROUND STATE

Bands of ${ }^{12} \mathrm{C}_{2} \mathrm{H}_{2}$

The bands $v_{3}$ and $v_{2}+v_{4}+v_{5}$ have been most recently reassigned by Lafferty and Thibault (2). A Fermi resonance given by Eq. (18) occurs between the upper states with the notable result that the intensities of the two bands are approximately the same; the band at the higher wave number is assigned as $v_{3^{*}}$

Both of these bands have been measured up to approximately $\mathrm{J}=50$ in the present work. It has been observed, in agreement with the results in Reference (2), that the rotational energies of the upper states, $0010^{0} 0$ and $0101^{1} 1^{1}$, cannot be expressed as $F^{\prime}(J)=B^{\prime} J(J+1)-D^{\prime} J^{2}(J+1)^{2}$. An additional term or terms was required to fit the observed combination differences $R(J)-P(J)$ and $R(J-1)+P(J)$. On the other hand, the use of additional terms did not improve the rms deviation of the fit for the ground state combination difference $R(J-1)-P(J+1)$; this demonstrates that the need for additional terms arises from effects in the upper states and not from any possible errors in calculating the dispersion curve. Lafferty found that the addition of an $\mathrm{H}^{2}$ terms, resulting in the expression for $\mathrm{P}$ - and R-branch line positions given below in Eq. (17), gave a satisfactory fit of the observed data.

$$
\begin{gathered}
v(m)=v_{0}+\left(B^{\prime}+B^{\prime \prime}\right) m+\left(B^{*}-B^{\prime \prime}-D^{\prime}+D^{\prime \prime}\right) m^{2}-\left(2 D^{\prime}+2 D^{\prime \prime}-H^{\prime}\right) m^{3} \\
-\left(D^{\prime}-D^{\prime \prime}-3 H^{\prime \prime}\right) m^{4}+3 H^{\prime} m^{5}+H^{\prime \prime} m^{6}
\end{gathered}
$$


The line positions (vacuum $\mathrm{cm}^{-1}$ ) calculated in this way are given in Table 8, along with the observed values. Table 9 compares the constants determined with those obtained by Lafferty, using the same kind of fit. The extent of agreement is good; although the calculated uncertainties of the constants do not overlap, these uncertainties are not in any sense absolute in that only random, and not systematic errors are included in such calculations. In particular, there is a shift of about $0.03 \mathrm{~cm}^{-1}$ between Lafferty's data and ours over most of the region measured, as is reflected in our values for the band centers given in Table 9. To help clarify this matter, the bands were measured with both HC1 and Co calibration; with each standard we obtained the same result for the band centers to within $\pm 0.002 \mathrm{~cm}^{-1}$. A singlepen system was used to eliminate pen lag. The acetylene data were recorded interspersed with HC1 or CO lines, which were recorded by changing the prism order. It is doubtful that this system would allow a band center error of $0.03 \mathrm{~cm}^{-1}$.

While the expression in Eq. (17) fits our data with an rms deviation which approximates the experimental uncertainty of measurement, the data is not sufficient to conclude that such a dependence is involved. Firstly, the term $3 \mathrm{H}^{5}$ is dominated at low J-values by the $\mathrm{m}^{4}$ and lower order terms and at high J-values by the $\mathrm{m}^{6}$ term, so that the data can be fitted to $v(\mathrm{~m})=\mathrm{A}+\mathrm{Bm}+\mathrm{Cm}^{2}+\mathrm{Dm}^{3}+\mathrm{Em}^{4}+\mathrm{Gm}^{6}$ about as well as they can to Eq. (17). Secondly, the addition of a seventhorder term did lower the standard deviation of fit slightly for our data. These two points indicate that functional dependence given by Eq. (17) is not justified by the data. In the absence of a theoret- 
TABLE 8

ROTATIONAL ANALYS IS OF THE BANDS $v_{3}$ AND $v_{2}+v_{4}+v_{5}\left(\mathrm{~cm}^{-1}\right)$

*denotes blend

\begin{tabular}{|c|c|c|c|c|c|c|c|c|}
\hline \multirow[b]{3}{*}{$\mathbf{J}$} & \multicolumn{4}{|c|}{$v_{3}$} & \multicolumn{4}{|c|}{$v_{2}+v_{4}+v_{5}$} \\
\hline & \multicolumn{2}{|c|}{$P(J)$} & \multicolumn{2}{|c|}{$R(J)$} & \multicolumn{2}{|c|}{$P(J)$} & \multicolumn{2}{|c|}{$R(J)$} \\
\hline & OBSERVED & $0-c$ & OBSERVED & $0-C$ & OBSERVED & $0-C$ & OBSERVED & $0-C$ \\
\hline 0 & & & 3297.16 & -0.01 & & & 3284.23 & 0.00 \\
\hline 1 & 3292.49 & +0.02 & 3299.51 & +0.01 & 3279.55 & +0.01 & 3286.56 & -0.01 \\
\hline 2 & 3290.13 & +0.02 & 3301.81 & -0.02 & 3277.20 & +0.02 & 3288.91 & +0.01 \\
\hline 3 & 3287.74 & 0.00 & 3304.14 & -0.01 & 3274.81 & 0.00 & 3291.21 & -0.01 \\
\hline 4 & 3285.40 & +0.04 & 3306.48 & +0.02 & 3272.43 & 0.00 & 3293.53 & 0.00 \\
\hline 5 & 3282.99 & +0.01 & 3308.75 & -0.01 & 3270.06 & +0.01 & 3295.82 & -0.01 \\
\hline 6 & 3280.59 & +0.01 & 3311.07 & +0.01 & 3267.67 & +0.01 & 3298.13 & +0.01 \\
\hline 7 & 3278.19 & +0.01 & 3313.34 & 0.00 & 3265.26 & 0.00 & 3300.40 & -0.01 \\
\hline 8 & 3275.77 & 0.00 & 3315.62 & +0.01 & 3262.84 & -0.01 & 3302.69 & 0.00 \\
\hline 9 & 3273.34 & -0.01 & 3317.86 & -0.01 & 3260.43 & 0.00 & 3304.95 & 0.00 \\
\hline
\end{tabular}


TABLE 8 (Continued)

\begin{tabular}{|c|c|c|c|c|c|c|c|c|}
\hline \multirow[b]{3}{*}{$J$} & \multicolumn{4}{|c|}{$v_{3}$} & \multicolumn{4}{|c|}{$v_{2}+v_{4}+v_{5}$} \\
\hline & \multicolumn{2}{|c|}{$P(J)$} & \multicolumn{2}{|c|}{$R(J)$} & \multicolumn{2}{|c|}{$P(J)$} & \multicolumn{2}{|c|}{$R(J)$} \\
\hline & OBSERVED & $0-C$ & OBSERVED & O-C & OBSERVED & $0-C$ & OBSERVED & $0-C$ \\
\hline 10 & 3270.90 & -0.02 & 3320.12 & -0.01 & 3258.00 & 0.00 & 3307.23 & +0.02 \\
\hline 11 & 3268.47 & -0.01 & 3322.39 & +0.02 & 3255.58 & +0.01 & 3309.45 & -0.01 \\
\hline 12 & 3266.02 & -0.01 & 3324.60 & 0.00 & 3253.12 & 0.00 & 3311.71 & +0.01 \\
\hline 13 & 3263.56 & 0.00 & 3326.82 & +0.01 & 3250.67 & 0.00 & 3313.92 & 0.00 \\
\hline 14 & 3261.09 & 0.00 & 3329.03 & +0.01 & 3248.20 & -0.01 & 3316.14 & 0.00 \\
\hline 15 & 3258.60 & -0.00 & 3331.23 & +0.02 & 3245.74 & 0.00 & 3318.35 & 0.00 \\
\hline 16 & 3256.11 & -0.01 & 3333.43 & $+0.04 *$ & 3243.24 & -0.02 & 3320.55 & +0.01 \\
\hline 17 & 3253.61 & 0.00 & 3335.57 & $+0.01 *$ & 3240.77 & 0.00 & 3322.74 & +0.01 \\
\hline 18 & 3251.09 & 0.00 & 3337.69 & $-0.02 *$ & $3238.29 *$ & +0.02 & 3324.92 & +0.02 \\
\hline 19 & 3248.58 & +0.02 & 3339.81 & $-0.03 *$ & $3235.74 *$ & -0.01 & 3327.05 & -0.01 \\
\hline 20 & 3246.04 & +0.02 & 3341.97 & +0.01 & 3233.25 & +0.02 & 3329.20 & -0.01 \\
\hline
\end{tabular}


TABLE 8 (Continued)

\begin{tabular}{|c|c|c|c|c|c|c|c|c|}
\hline \multirow[b]{3}{*}{$\mathrm{J}$} & \multicolumn{4}{|c|}{$v_{3}$} & \multicolumn{4}{|c|}{$v_{2}+v_{4}+v_{5}$} \\
\hline & \multicolumn{2}{|c|}{$P(J)$} & \multicolumn{2}{|c|}{$R(J)$} & \multicolumn{2}{|c|}{$P(J)$} & \multicolumn{2}{|c|}{$R(J)$} \\
\hline & OBSERVED & $\mathrm{O}-\mathrm{C}$ & OBSERVED & $0-C$ & OBSERVED & O-C & OBSERVED & O-C \\
\hline 21 & 3243.47 & +0.01 & 3344.06 & -0.01 & 3230.70 & 0.00 & 3331.32 & -0.02 \\
\hline 22 & 3240.88 & -0.01 & 3346.15 & 0.00 & 3228.16 & 0.00 & 3333.43 & $-0.03 *$ \\
\hline 23 & $3238.29 *$ & -0.02 & 3348.22 & -0.01 & 3225.60 & 0.00 & 3335.57 & $0.00 *$ \\
\hline 24 & $3235.74 *$ & +0.03 & 3350.26 & -0.02 & 3223.04 & 0.00 & 3337.69 & $+0.02 *$ \\
\hline 25 & 3233.09 & -0.01 & 3352.30 & -0.01 & 3220.48 & +0.02 & 3339.81 & $+0.06 *$ \\
\hline 26 & 3230.47 & 0.00 & 3354.33 & 0.00 & 3217.87 & 0.00 & 3341.83 & +0.01 \\
\hline 27 & 3227.82 & 0.00 & 3356.31 & -0.02 & 3215.26 & -0.01 & 3343.88 & +0.01 \\
\hline 28 & 3225.16 & 0.00 & 3358.28 & -0.03 & 3212.66 & 0.00 & 3345.91 & 0.00 \\
\hline 29 & 3222.48 & 0.00 & 3360.26 & -0.01 & 3210.04 & +0.01 & 3347.93 & 0.00 \\
\hline 30 & 3219.78 & -0.01 & 3362.21 & 0.00 & 3207.39 & 0.00 & 3349.93 & -0.01 \\
\hline 31 & 3217.09 & +0.01 & 3364.19 & +0.06 & 3204.74 & 0.00 & 3351.94 & +0.01 \\
\hline
\end{tabular}


TABLE 8 (Continued)

\begin{tabular}{|c|c|c|c|c|c|c|c|c|}
\hline \multirow[b]{3}{*}{$\mathrm{J}$} & \multirow{2}{*}{\multicolumn{2}{|c|}{$P(J)$}} & & & \multicolumn{4}{|c|}{$v_{2}+v_{4}+v_{5}$} \\
\hline & & & R( & & $\mathbf{P}$ & & $\mathrm{R}($ & \\
\hline & OBSERVED & $0-c$ & OBSERVED & $0-c$ & OBSERVED & $0-c$ & OBSERVED & $0-C$ \\
\hline 32 & 3214.36 & 0.00 & 3366.04 & 0.00 & 3202.08 & 0.00 & 3353.90 & -0.01 \\
\hline 33 & 3211.61 & 0.00 & 3367.91 & - 0.01 & 3199.40 & 0.00 & 3355.86 & -0.01 \\
\hline 34 & 3208.84 & -0.02 & 3369.78 & -0.01 & 3196.68 & -0.03 & 3357.82 & +0.01 \\
\hline 35 & 3206.08 & 0.00 & 3371.65 & +0.02 & 3194.00 & 0.00 & 3359.74 & 0.00 \\
\hline 36 & 3203.35 & +0.06 & 3373.47 & +0.01 & 3191.29 & 0.00 & 3361.66 & +0.01 \\
\hline 37 & 3200.49 & 0.00 & 3375.29 & +0.01 & 3188.56 & 0.00 & 3363.56 & +0.01 \\
\hline 38 & & & 3377.09 & +0.02 & 3185.82 & 0.00 & 3365.43 & 0.00 \\
\hline 39 & 3194.82 & -0.01 & 3378.87 & +0.01 & 3183.07 & +0.01 & 3367.29 & 0.00 \\
\hline 40 & 3191.98 & -0.01 & 3380.63 & +0.01 & 3180.28 & -0.01 & 3369.15 & +0.01 \\
\hline 41 & 3189.13 & +0.01 & 3382.36 & -0.02 & $3177.52 *$ & +0.01 & 3370.96 & -0.01 \\
\hline 42 & 3186.24 & -0.01 & 3384.10 & -0.02 & $3174.71 *$ & -0.01 & 3372.84 & +0.06 \\
\hline
\end{tabular}


TABLE 8 (Continued)

\begin{tabular}{|c|c|c|c|c|c|c|c|c|}
\hline \multirow[b]{2}{*}{$\mathbf{J}$} & \multicolumn{2}{|c|}{$P(J)$} & $\mathrm{R}$ & & \multicolumn{4}{|c|}{$v_{2}+v_{4}+v_{5}$} \\
\hline & OBSERVED & O-C & OBSERVED & $0-C$ & OBSERVED & $0-C$ & OBSERVED & $0-c$ \\
\hline 43 & 3183.34 & -0.02 & 3385.89 & +0.03 & & & 3374.57 & -0.01 \\
\hline 44 & & & 3387.54 & -0.04 & & & & \\
\hline 45 & $3177.52 *$ & -0.04 & 3389.24 & -0.07 & 3166.27 & 0.00 & 3378.11 & -0.02 \\
\hline 46 & $3174.71 *$ & +0.06 & & & & & & \\
\hline 47 & & & 3392.72 & -0.03 & & & 3381.65 & +0.02 \\
\hline 48 & . & & 3394.47 & 0.00 & & & & ' \\
\hline 49 & & & 3396.18 & -0.03 & & & & \\
\hline 50 & & & 3398.00 & +0.05 & & & $:$ & \\
\hline 51 & & & & & & & & \\
\hline 52 & & & 3401.49 & 0.00 & & & & \\
\hline
\end{tabular}


TABLE 9

CONSTANTS DERIVED FROM THE BANDS AT 3 MICRONS

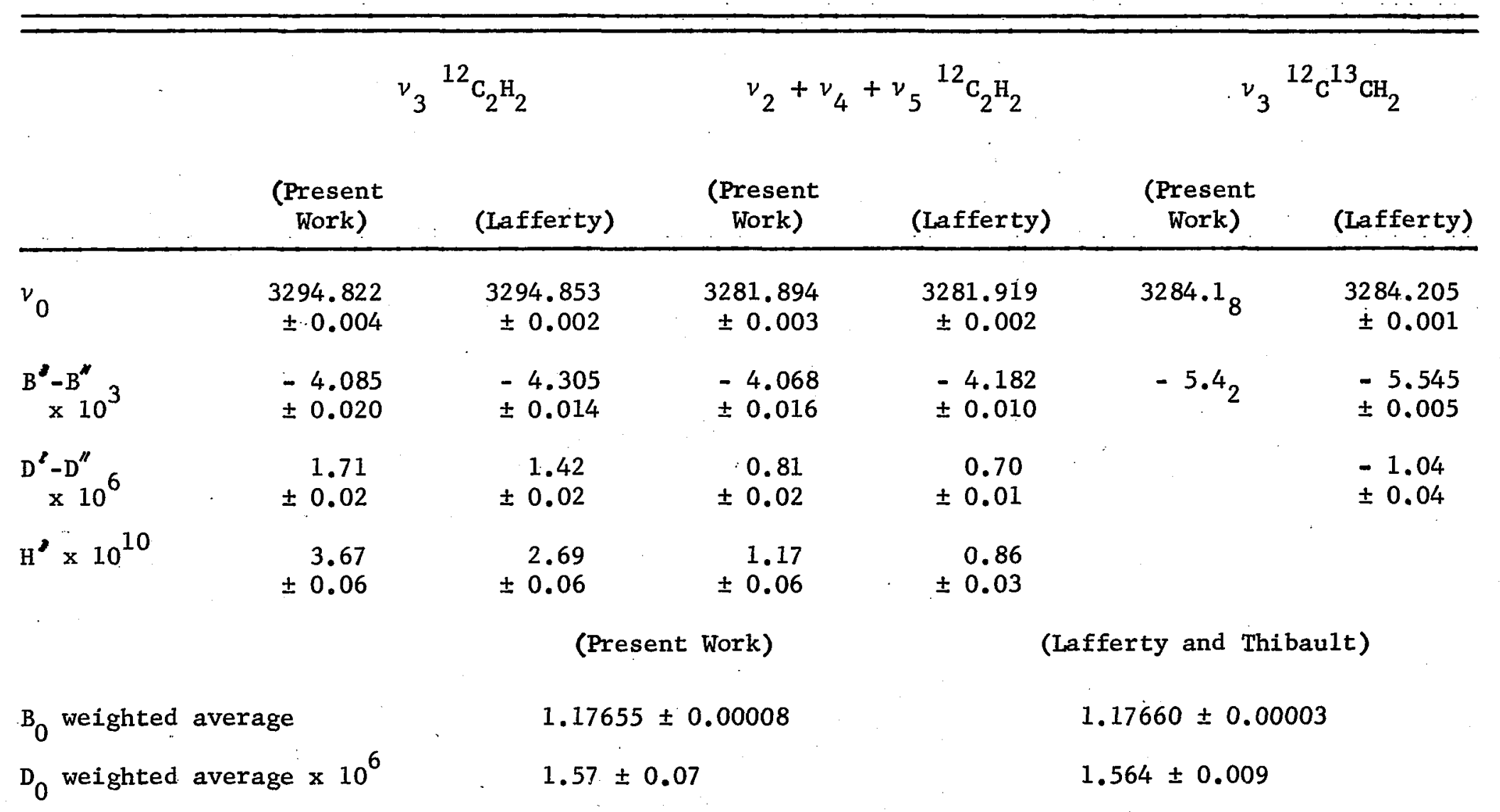


ically derived expression for the higher-order rotational effects of the Fermi resonance between the two upper states in question, it seems premature to represent the data by an expression involving an $\mathrm{H}^{*}$ term, even an $\mathrm{H}^{\prime}$ (effective).

The data do clearly show that the effect present in the two bands under discussion can be approximated very well by $F^{\prime}(J)=B^{t} J(J+1)-D^{2} J^{2}(J+1)^{2}+k J^{6}$. This is shown in Figs. 9 and 10 . Bands of ${ }^{12} \mathrm{C}^{13} \mathrm{CH}_{2}$ :

The bands $v_{3}$ and $v_{2}+v_{4}+v_{5}$ of this molecule were observed, although an unenriched sample of acetylene was used. It was possible to measure $v_{3}$ up to about $\mathrm{J}=20$ for both $\mathrm{P}$ - and $\mathrm{R}$ - branches, a1though the R-branch lines were more often blended. Constants derived from this band are given in Table 9. While our data for this species are less extensive than that which Lafferty obtained with an enriched sample, the determination of the band center in our work was sufficient1y accurate to show the $0.03 \mathrm{~cm}^{-1}$ shift discussed above. $v_{2}+v_{4}+v_{5}$ is weaker than $v_{3}$ and could only be observed on the long wavelength side of the 3 micron bands, where it was not as badly overlapped. $P(7)$ through $P(34)$ of this band were observed. No band center is given for this band in Table 9 because of the extrapolation it would require from our data. 
Figure 9

$v(\mathrm{~m})$ versus $\mathrm{m}$ for $v_{2}+v_{4}+v_{5}$ 


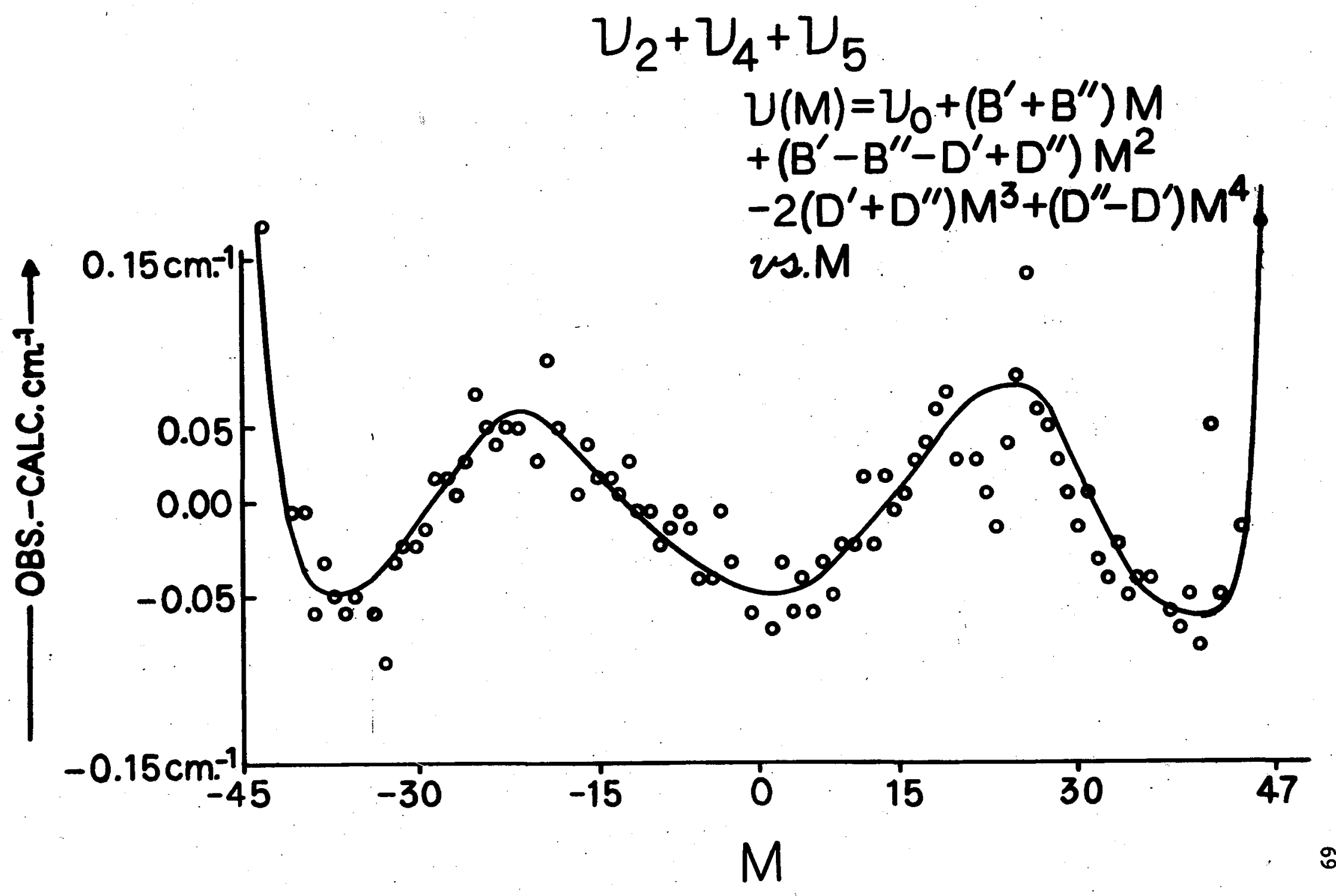


Figure 10

$R(J=1)+P(J)$ versus $J$ for $v_{3}$ 


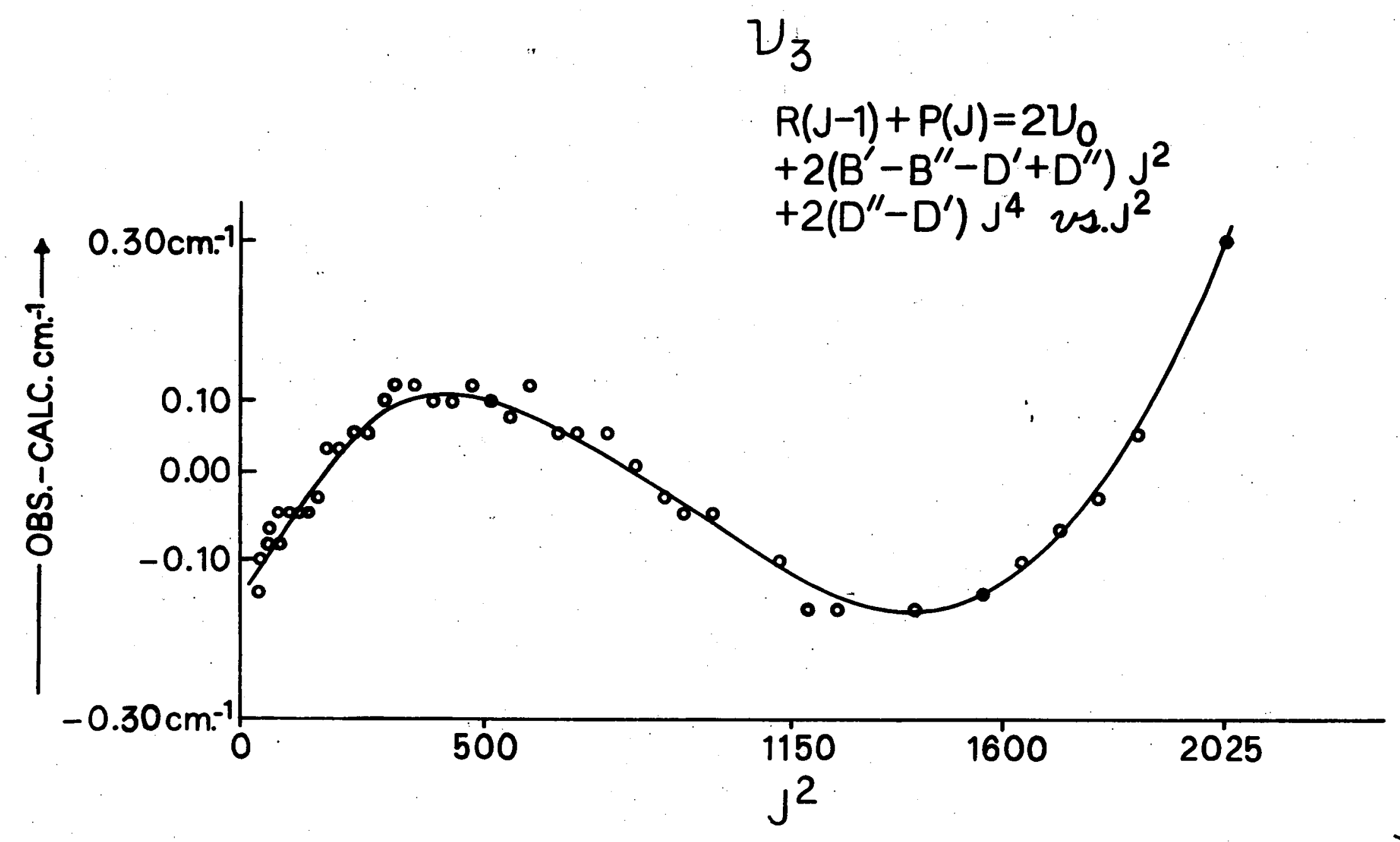




\section{TRANSITIONS FROM OTHER LEVELS: "HOT" BANDS}

Vibrational Analysis:

The two groups of resonating triads in Fig. 8 are of particular theoretical interest in that each should show both Fermi resonance of the form given in Eq. (18) (2).

$$
\begin{aligned}
& \left(v_{1} ; v_{2} ; v_{3} ; v_{4}, l_{4} ; v_{5}, l_{5} \| v_{1} ; v_{2}-1 ; v_{3}+1 ; v_{4}-1, \ell_{4} \mp 1 ;\right. \\
& \left.v_{5}-1, l_{5} \pm 1\right)=(-\gamma / 8)\left[v_{2}\left(v_{3}+1\right)\left(v_{4} \pm l_{4}\right)\left(v_{5} \mp l_{5}\right)\right]^{1 / 2}
\end{aligned}
$$

and vibrational l-type resonance of the form (6) given in Eq. (19)

$$
\begin{aligned}
& \left(v_{4}, \ell_{4} ; v_{5}, l_{5} \| v_{4}, l_{4} \pm 2 ; v_{5}, l_{5} \mp 2\right)= \\
& 1 / 4 r_{45}\left[\left(v_{4} \pm \ell_{4}+2\right)\left(v_{4} \mp l_{4}\right)\left(v_{5} \pm l_{5}+2\right)\left(v_{5} \pm l_{5}\right)\right]^{1 / 2}
\end{aligned}
$$

The resulting matrix for the energy levels in the first group in Fig. 8 is given in Fig. 11; the same matrix describes the energy levels in the second group in Fig. 8 if the levels $0102^{0} 1^{1}, 0011^{1} 0^{0}, 0102^{2} 1^{1}$ are replaced by $0101^{1} 2^{0}, 0010^{0} 1^{1}$, and $0101^{1} 2^{2}$, respectively. Here $E_{1}, E_{2}$, and $E_{3}$ are the unperturbed energy levels of the states designated by the quantum numbers given.

$\mathrm{E}_{1}, \mathrm{E}_{2}$, and $\mathrm{E}_{3}$ are calculated numerically below by use of the most recently determined values of the vibrational constants involved $(3,4,5)$; gamma is known numerically from the work of Lafferty and 


\section{Figure 11}

Submatrix Pertaining to Transitions at 3 Microns 


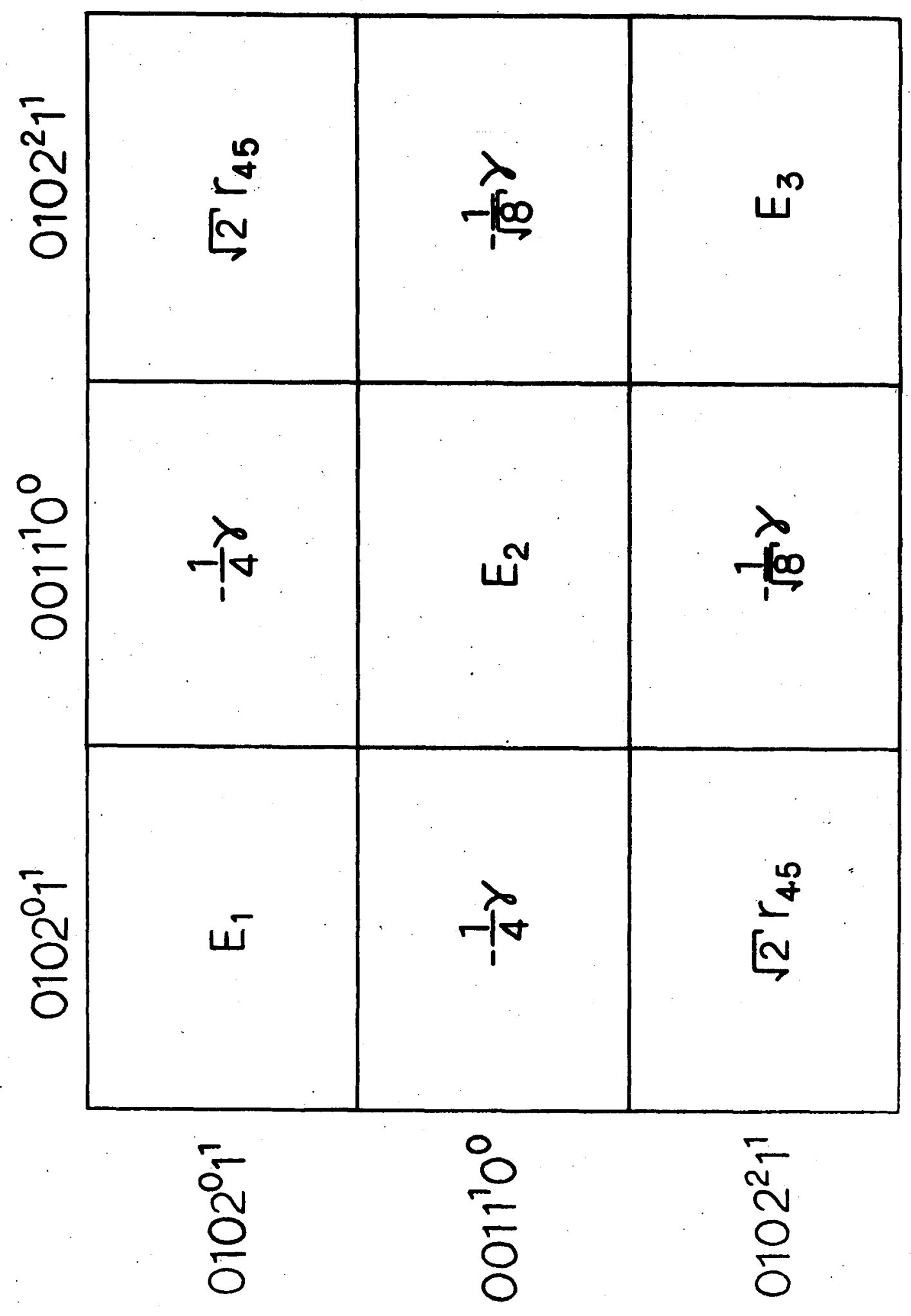


Thibault (2), and $r_{45}$ was obtained in our last paper of this series $(15,16)$. These values allow us to numerically diagonalize the matrix in Fig. 11. Since earlier calculations (6) (see Chapter III) yielded two alternate sets of values for the constants $g_{45}, x_{45}$, and $r_{45}$, this numerical diagonalization was performed with the values of $E_{1}, E_{3}$, and $r_{45}$ calculated from each set.

Set A

$$
\begin{aligned}
& g_{45}=7.92 \\
& x_{45}=-3.45 \\
& r_{45}=-3.66 \\
& g_{44}=-0.22
\end{aligned}
$$

Set B

$$
\begin{aligned}
& g_{45}=6.35 \\
& x_{45}=-1.88 \\
& r_{45}=-6.80 \\
& g_{44}=2.13
\end{aligned}
$$

Using the values given above, together with values of energy levels determined directly from observed transitions $(2,3,4,15)$ by means of the Ritz Combination Principle, the following is obtained (other constants from Ref. 3):

$$
\begin{aligned}
E_{1}= & 0102{ }^{0} 1 \text { (unperturbed) }=\left(v_{2}\right)+\left(2 v_{4}^{2}\right)+\left(v_{5}\right)-4 g_{44} \\
& +2 x_{24}+2 x_{45}+x_{25}=1973.99+1235.07+730.34+0.88 \\
& \therefore-25.40-6.90-1.38=3906.60 \quad \text { Set } A \\
= & 1973.99+1235.07+730.34-8.52-25.40-3.76-1.38 \\
= & 3900.34 \quad \text { Set B }
\end{aligned}
$$


$\mathrm{E}_{2}=0011^{1} 0^{0}$ (unperturbed) $=\left(v_{3} \underset{\text { unperturbed }}{)}+\left(v_{4}\right)+\mathrm{x}_{34}\right.$ $=3288.66+612.88-8.19=3893.35$

$$
\begin{aligned}
\mathrm{E}_{3}= & 0102^{2} 1^{1} \text { (unperturbed pi state) }=\left(v_{2}\right)+\left(2 v_{4}^{2}\right)+\left(v_{5}\right) \\
& +2 \mathrm{x}_{24}+2 \mathrm{x}_{45}+\mathrm{x}_{25}-2 \mathrm{~g}_{45}=1973.99+1235.07+730.34 \\
& -25.40-6.90-1.38-15.84=3888.88 \quad \text { Set } \mathrm{A} \\
= & 1973.99+1235.07+730.34-25.40-3.76-1.38-12.70 \\
= & 3896.16 \quad \text { Set B }
\end{aligned}
$$

Therefore, for these levels, the matrix in Fig. 11 becomes

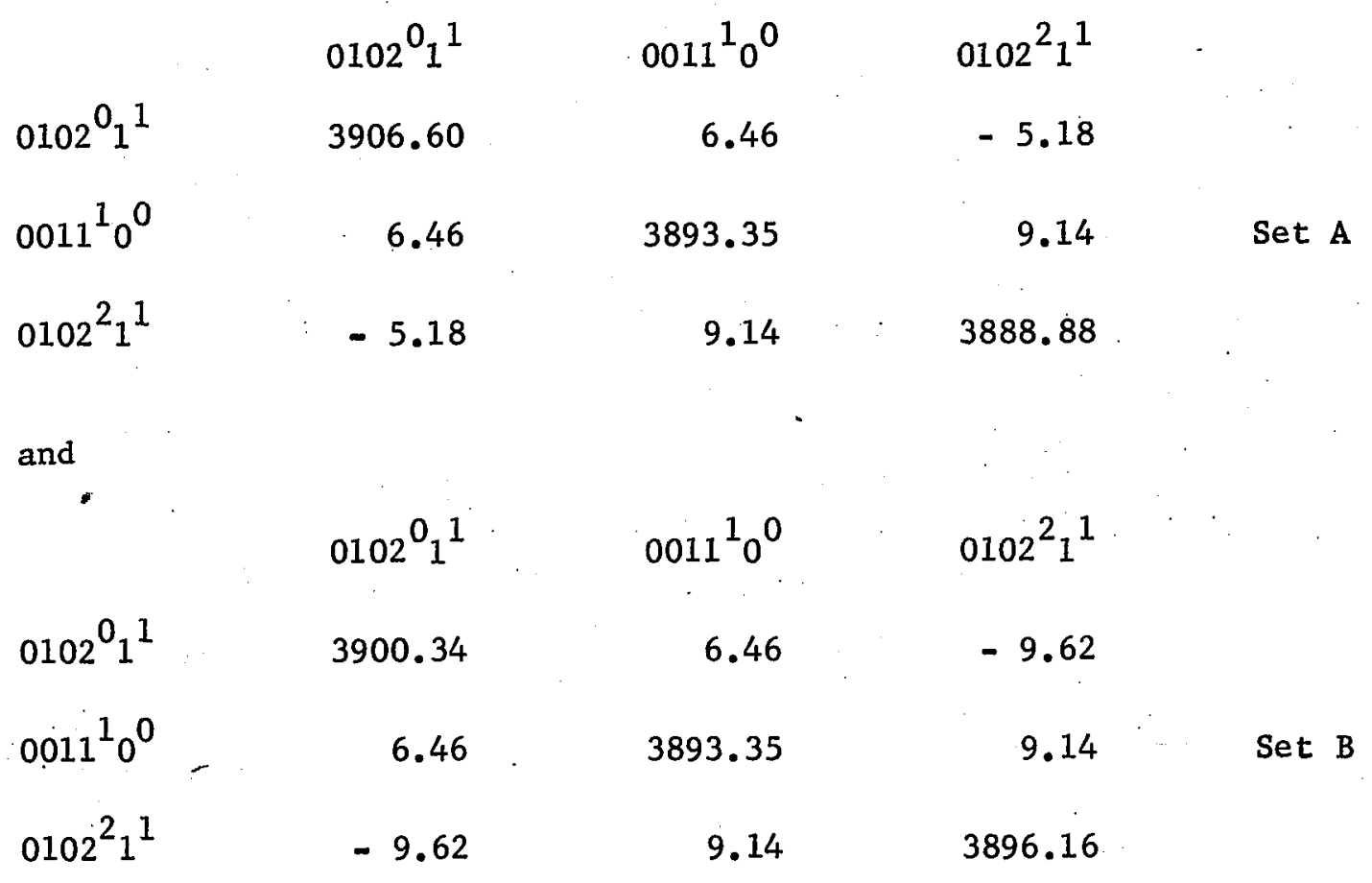


Upon diagonalization, these matrices yield the perturbed energy levels compared below with those determined from observed transitions by means of the Ritz Combination Principle:

$\begin{array}{ccc}\text { Set A } & \text { Set B } & \text { "Observed" } \\ 3909.35 & 3908.15 & \\ 3900.17 & 3902.27 & 3898.32 \\ 3879.31 & 3879.43 & 3882.63\end{array}$

Set $A$ is seen to yield slightly better agreement with the "observed" values. If the value of gamma is now changed from Lafferty's value of $25 \mathrm{~cm}^{-1}$ to $20 \mathrm{~cm}^{-1}$, one obtains:

$\begin{array}{ccc}\text { Set A } & \text { "Observed" } & \text { Set B } \\ 3908.73 & - & 3908.12 \\ 3898.44 & 3898.32 & 3899.84 \\ 3881.66 & 3882.63 & 3881.89\end{array}$

A similar calculation is performed below for the other resonating triad:

$$
\begin{aligned}
\mathrm{E}_{1}= & 0101^{1} 2^{0} \text { (unperturbed) }=\left(v_{2}\right)+\left(v_{4}\right)+\left(2 v_{5}{ }^{0}\right)+\mathbf{x}_{24}+2 \mathrm{x}_{25} \\
& +2 \mathrm{x}_{45} \\
= & 1973.99+612.88+1449.15-12.70-2.76-6.90=4013.66 \\
= & \text { Set } \mathrm{A}
\end{aligned}
$$




$$
\begin{aligned}
\mathrm{E}_{2} & =0010^{0} 1^{1} \text { (unperturbed) }=\left(v_{3}\right)+\left(v_{5}\right)+\mathbf{x}_{35} \\
& =3288.66+730.34-8.68=4010.32
\end{aligned}
$$

$$
\begin{aligned}
E_{3}= & 0101^{1} 2^{2} \text { (unperturbed pi state) }=\left(v_{2}\right)+\left(v_{4}\right)+\left(2 v_{5}^{2}\right) \\
& +x_{24}+2 x_{25}+2 x_{45}-2 g_{45}=1973.99+612.88+1462.72 \\
& -12.70-2.76-6.90-15.84=4011.39 \quad \text { Set } A \\
= & 1973.99+612.88+1462.72-12.70-2.76-3.76-12.70 \\
= & 4017.67 \quad \text { Set B }
\end{aligned}
$$

Therefore, for these levels, matrix I becomes:

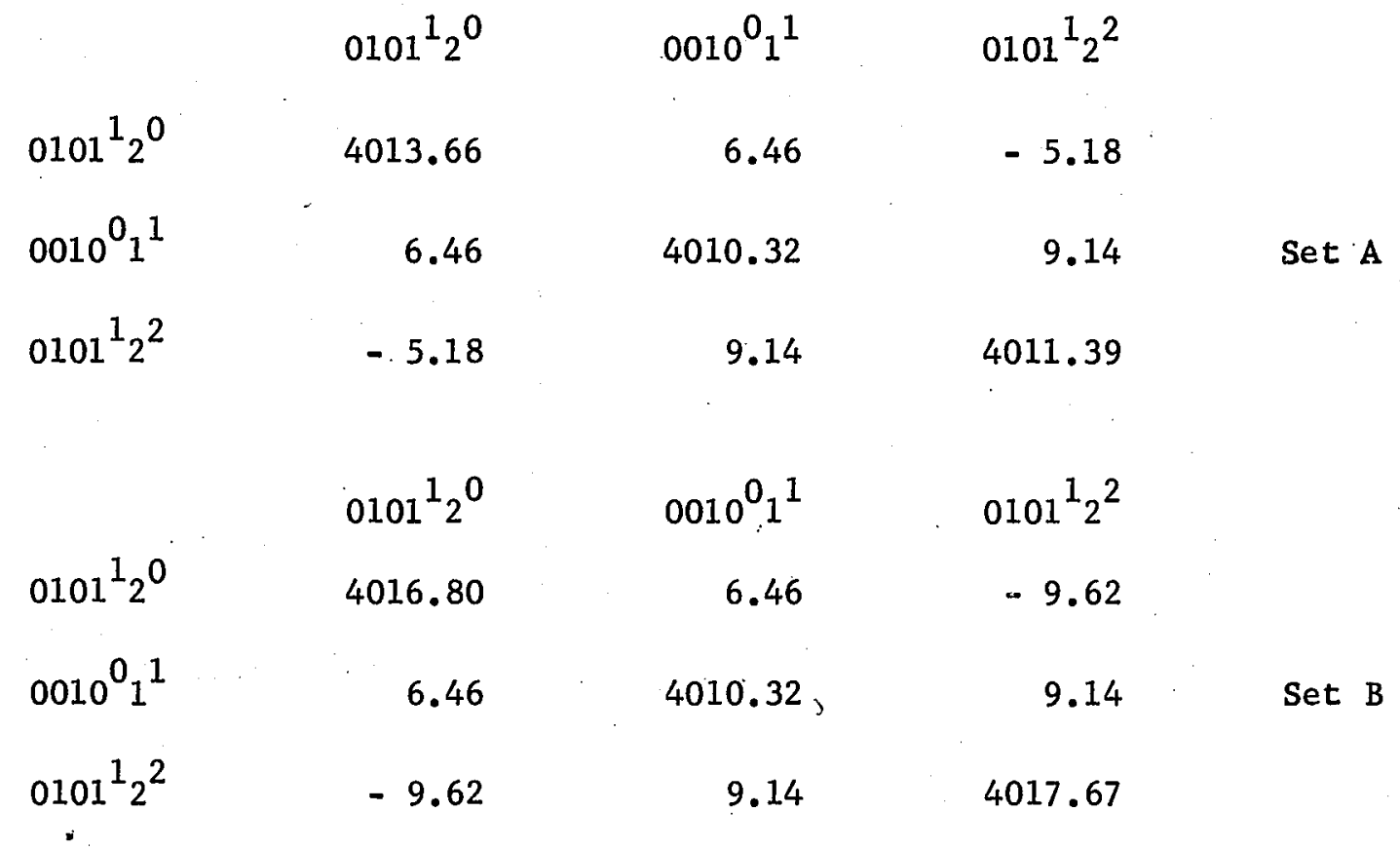

Upon diagonalization these matrices yield the perturbed energy levels compared below with those determined from observed transitions from the Ritz Combination Principle: 


$\begin{array}{ccc}\text { Set A } & \text { Set B } & \text { "Observed" } \\ 4020.17 & 4043.91 & - \\ 4017.72 & 4026.64 & 4016.71 \\ 3997.48 & 4004.24 & 3999.89\end{array}$

As with the triad involving $0011^{1} 0^{0}$, it is again seen that a better fit is obtained using the vibrational constants in Set A. When the value of gamma is again reduced from Lafferty's value of $25 \mathrm{~cm}^{-1}$ to $20 \mathrm{~cm}^{-1}$, the results shown below are obtained:

$\begin{array}{ccc}\text { Set A } & \text { "Observed" } & \text { Set B } \\ 4018.12 & - & 4042.56 \\ 4017.26 & 4016.71 & 4026.71 \\ 3999.99 & 3999.89 & 4017.67\end{array}$

As can be seen from the above discussion, the vibrational constants listed in Set A give a very nice fit to those four levels determined from observed transitions when the Fermi resonance parameter gamma is taken as $20 \mathrm{~cm}^{-1}$; this allows a choice between the two sets of vibrational constants and a determination of which set is most nearly correct, it allows a determination of the gamma, and it allows the assignment of the upper states of the observed hot bands in the three micron region. It appears that the vibrational calculations here are somewhat more sensitive to the value of gamma than were Lafferty's calculations; because of this, the value derived here more nearly approximates the true value. The constants determined from the dis- 
cussion above are listed below with observed and calculated vibrational levels:

$\begin{array}{lccc}\text { State } & \begin{array}{c}\text { Energy Leve1 } \\ \left(\mathrm{cm}^{-1}\right)\end{array} & \text { "Observed" } & 0-\mathrm{C} \\ 0101^{1} 2^{2} & 4017.26 & 4016.71 & -0.55 \\ 0010^{0}{ }^{1} & 3999.99 & 3999.89 & +0.10 \\ 0102^{2}{ }_{1}^{1} & 3881.66 & 3882.63 & -0.97 \\ 0011^{1} 0^{0} & 3898.44 & 3898.32 & +0.12 \\ \mathrm{x}_{45}=-3.45 & \mathrm{~g}_{45}=7.92 & \mathrm{r}_{45}=-3.66 \quad \mathrm{~g}_{44}=-0.22 & \gamma=20\end{array}$

It would seem that the above agreement of calculated and observed values is reasonable in view of the uncertainty in the values of some of the anharmonic constants involved in the calculation. $x_{34}$ and $x_{35}$ are not known with great accuracy, and this leads to an uncertainty in the unperturbed values of $\mathrm{E}_{2}\left(0011^{1} 0^{0}\right.$, and $\left.0010^{0} 1^{1}\right)$, hence to errors in the calculated perturbed values. Two questions seem to remain with regard to the above discussion, however. The first is the difference between the value of $\mathrm{g}_{44}$ and that of $1.36 \mathrm{~cm}^{-1}$ determined by Innes from the levels $0001^{1} 0^{0}, 0002^{2} 0^{0}, 0003^{1} 0$, and $0004^{0} 0^{0}$. With regard to this matter, it should be pointed out that Innes' four levels do not involve a pair such as $0002^{0} 0^{0}$ and $0002^{2} 0^{0}$ or $0004^{0} 0^{0}$ and $0004^{2} 0^{0}$ and are consequently relatively insensitive to the calculation of $\mathrm{g}_{44}$; moreover, the consistency of his values for these levels may be due in part to the fact that they provide a system of three equations in three unknowns 
$\left(\omega_{4}^{0}, x_{44}, g_{44}\right)$, and not four equations, since that for $0004^{0} 0^{0}$ is linearly dependent upon those for $0001^{1} 0^{0}$ and $0003^{1} 0^{0}$. For these reasons, the two values $\mathrm{g}_{44}=-0.22$ and $+1.36 \mathrm{~cm}^{-1}$ are perhaps equally inaccurate or equally accurate. The second point is that the transitions to the uppermost level of each resonating triad were not observed. For the triad involving $0011^{1} 0^{0}$ this is quite understandable. since the unperturbed energy level $0102^{0} 1^{1}$ is quite a bit higher than the other two; the operator which diagonalizes these three levels shows clearly that the mixing of $0102^{0} 1^{1}$ with $0011^{1} 0^{0}$ is rather slight, so that the naturally strong intensity of $v_{3}+v_{4}-v_{4}$ is not expected to be shared with $v_{2}+2 v_{4}^{0}+v_{5}-v_{4}$. On the other hand, the zero-order wave functions designated by $0101^{1} 2^{0}, 0101^{1} 2^{2}$, and $0010^{0} 1^{1}$ are all quite thoroughly mixed, owing to the extreme nearness of the unperturbed levels. Therefore, the fact that no band was observed which could be interpreted as $v_{2}+v_{4}+2 v_{5}^{0}-v_{5}$ is puzzling.

Rotationa1 Analysis:

Rotational assignments for the four $I$ - II transitions observed are given in Tables 10 through 13, along with the derived constants. Calculated values were determined by fitting fourth-order curves to the wave numbers of each subband as a function of m-number. This fit yields the parameters $B$ and $D$. In addition, the l-type splittings of each rotational line were fit to $\Delta y=\left(\mathrm{q}^{\prime \prime}+\mathrm{q}^{\prime \prime}\right) \mathrm{m}+\left(\mathrm{q}^{\prime \prime}-\mathrm{q}^{\prime \prime}\right) \mathrm{m}^{2}$, where $\mathrm{q}$ is the rotational -type doubling constant defined by Herzberg (1) and used by Plyler et al. (12). Some deviation of the l-type splittings from the quadratic dependence given above seems to be in evidence, 
TABLE 10

ROTATIONAL ANALYSIS OF THE BAND $v_{3}+v_{4}-v_{4}$

Q-branch at 3285.44 (A11 units $\mathrm{cm}^{-1}$ )

\begin{tabular}{|c|c|c|c|c|c|c|}
\hline $\mathbf{J}$ & $P(J)^{a}$ & $\begin{array}{l}\text { O-C } \\
\text { ave. }\end{array}$ & $R(J)$ & $0-C$ & $R(J)$ & $0-C$ \\
\hline 1 & & & 3290.13 & 0.00 & & \\
\hline 2 & 3280.71 & -0.02 & & & 3292.49 & 0.00 \\
\hline 3 & 3278.34 & -0.02 & 3294.79 & +0.02 & & \\
\hline 4 & 3275.96 & -0.02 & & & 3297.16 & +0.03 \\
\hline 5 & 3273.60 & +0.01 & 3299.39 & +0.02 & & \\
\hline 6 & 3271.18 & -0.01 & & & 3301.71 & -0.03 \\
\hline 7 & 3268.79 & 0.00 & 3303.94 & +0.01 & & \\
\hline 8 & 3266.36 & -0.01 & 3306.17 & -0.03 & 3306.36 & +0.04 \\
\hline 9 & 3253.96 & +0.02 & 3308.47 & +0.02 & & \\
\hline 10 & 3261.50 & -0.01 & 3310.70 & 0.00 & 3310.88 & +0.03 \\
\hline 11 & 3259.08 & +0.01 & 3312.92 & -0.01 & 3313.06 & -0.05 \\
\hline 12 & $3256: 62$ & +0.01 & 3315.15 & 0.00 & 3315.36 & +0.01 \\
\hline 13 & 3254.17 & +0.02 & 3317.32 & -0.04 & 3317.59 & 0.00 \\
\hline 14 & 3251.69 & +0.01 & 3319.53 & -0.03 & 3319.80 & -0.01 \\
\hline 15 & 3249.23 & +0.03 & 3321.76 & +0.01 & 3322.05 & +0.03 \\
\hline 16 & 3246.70 & 0.00 & 3323.93 & +0.01 & 3324.23 & +0.01 \\
\hline 17 & 3244.22 & +0.02 & 3326.08 & 0.00 & 3326.43 & +0.02 \\
\hline 18 & 3241.70 & +0.01 & 3328.24 & +0.01 & 3328.60 & +0.01 \\
\hline 19 & 3239.17 & 0.00 & 3330.36 & 0.00 & 3330.79 & +0.04 \\
\hline
\end{tabular}


TABLE 10 (Continued)

\begin{tabular}{|c|c|c|c|c|c|c|}
\hline $\mathbf{J}$ & $P(J)$ & $\begin{array}{l}0-C \\
\text { ave. }\end{array}$ & $R(J)$ & $0-C$ & $R(J)$ & O-C \\
\hline 20 & 3236.63 & 0.00 & 3332.47 & -0.01 & 3332.91 & 0.00 \\
\hline 21 & 3234.09 & +0.01 & 3334.61 & +0.02 & 335.03 & -0.01 \\
\hline 22 & 3231.52 & 0.00 & 3336.72 & +0.04 & 3337.18 & +0.02 \\
\hline 23 & 3228.95 & +0.01 & 3338.73 & -0.02 & 3339.28 & +0.01 \\
\hline 24 & 3226.35 & -0.01 & 3340.85 & +0.04 & 3341.33 & -0.03 \\
\hline 25 & 3223.75 & -0.01 & 3342.85 & -0.01 & 3343.42 & -0.02 \\
\hline 26 & 3221.10 & -0.04 & 3344.85 & -0.03 & 3345.49 & -0.01 \\
\hline 27 & 3218.50 & -0.01 & 3346.85 & -0.04 & 3347.56 & +0.02 \\
\hline 28 & 3215.88 & +0.01 & 3348.88 & 0.00 & 3349.56 & 0.00 \\
\hline 29 & 3213.15 & -0.06 & 3350.84 & -0.01 & 3351.57 & $=0.00$ \\
\hline 30 & 3210.50 & -0.04 & 3352.78 & -0.02 & 3353.56 & 0.00 \\
\hline 31 & 3207.90 & +0.05 & 3354.73 & 0.00 & 3355.52 & 0.00 \\
\hline 32 & 3205.20 & +0.07 & 3356.64 & 0.00 & 3357.47 & 0.00 \\
\hline 33 & 3202.38 & -0.02 & 3358.57 & +0.04 & 3359.38 & -0.01 \\
\hline 34 & & & 3360.53 & +0.13 & 3361.29 & 0.00 \\
\hline 35 & & & & & & \\
\hline 36 & & & & & 3365.15 & +0.13 \\
\hline 37 & & & & & 3366.89 & +0.04 \\
\hline & CONS? & DERI & $\begin{array}{l}\text { band ce } \\
\left(B^{\prime}-B^{\prime \prime}\right) \\
\left(D^{\prime}-D^{\prime \prime}\right) \\
.55 \times 1.0\end{array}$ & \multicolumn{3}{|c|}{$\begin{array}{l}=3285.455 \pm 0.006 \\
=-4.21 \times 10^{-3} \\
=0.96 \times 10^{-6} \\
\quad q_{4}=q^{\prime \prime}=5.14 \times 10^{-3}\end{array}$} \\
\hline
\end{tabular}

$a_{\ell \text {-doublets unresolved }}$ 


\section{TABLE 11}

ROTATIONAL ANALYSIS OF THE BAND $v_{3}+v_{5}-v_{5}$

\begin{tabular}{|c|c|c|c|c|c|c|c|c|}
\hline $\mathrm{J}$ & $P(J)$ & $0-C$ & $P(J)$ & $0-c$ & $R(J)$ & $0-\mathrm{C}$ & $R(J)$ & $0-C$ \\
\hline 1 & & & & & & 3274.22 & $-0.03^{a}$ & \\
\hline 2 & & 3264.86 & $+0.02^{\mathrm{a}}$ & & ' & 3276.54 & -0.04 & \\
\hline 3 & . & 3262.45 & -0.01 & & & & 3278.86 & -0.01 \\
\hline 4 & & 3260.05 & -0.03 & & 3281.24 & -0.02 & 3281.16 & -0.01 \\
\hline 5 & & $3257.69^{\circ}$ & 0.00 & & 3283.57 & -0.01 & 3283.44 & -0.02 \\
\hline 6 & & 3255.30 & +0.01 & & 3285.88 & -0.01 & 3285.73 & -0.01 \\
\hline 7 & & 3252.89 & 0.00 & & 3288.24 & +0.05 & 3287.99 & -0.02 \\
\hline 8 & & 3250.52 & +0.05 & & 3290.51 & +0.03 & 3290.23 & -0.04 \\
\hline 9 & . & 3248.09 & +0.04 & & 3292.79 & +0.02 & 3292.49 & -0.03 \\
\hline 10 & & & & & 3295.08 & +0.04 & 3294.79 & +0.03 \\
\hline 11 & & & & & & & 3297.03 & +0.04 \\
\hline 12 & & & & & & & 3299.22 & +0.01 \\
\hline 13 & & & & & & & 3301.42 & +0.01 \\
\hline
\end{tabular}


TABLE 11 (Continued)

\begin{tabular}{|c|c|c|c|c|c|c|c|c|}
\hline $\mathrm{J}$ & $P(J)$ & $0-C$ & $P(J)$ & $0-c$ & $R(J)$ & $0-C$ & $R(J)$ & $0-c$ \\
\hline 14 & 3235.92 & +0.01 & & & & & 3303.64 & +0.03 \\
\hline 15 & 3233.45 & -0.01 & & & & & 3305.82 & +0.02 \\
\hline 16 & 3231.01 & +0.01 & & & & & 3308.01 & +0.03 \\
\hline 17 & 3228.56 & +0.03 & & & & & 3310.16 & +0.01 \\
\hline 18 & 3226.10 & +0.05 & & & & & 3312.30 & 0.00 \\
\hline 19 & 3223.54 & -0.03 & & & & & 3314.45 & 0.00 \\
\hline 20 & 3221.10 & +0.02 & & & & & 3316.57 & -0.02 \\
\hline 21 & 3218.57 & -0.01 & 3217.93 & +0.01 & & & 3318.68 & -0.03 \\
\hline 22 & 3216.08 & +0.01 & . & & 3321.51 & +0.02 & & \\
\hline 23 & 3213.55 & 0.00 & 3212.72 & -0.01 & 3323.63 & +0.02 & & \\
\hline 24 & 3211.04 & +0.02 & & & 3325.73 & +0.01 & & \\
\hline 25 & 3208.48 & 0.00 & 3207.51 & +0.01 & 3327.77 & -0.03 & & \\
\hline 26 & 3205.93 & -0.01 & 3204.86 & -0.01 & 3329.86 & -0.01 & & \\
\hline 27 & 3203.35 & -0.03 & 3202.23 & +0.01 & 3331.90 & -0.02 & & \\
\hline 28 & 3200.81 & 0.00 & 3199.56 & 0.00 & 3333.96 & 0.00 & & \\
\hline
\end{tabular}


TABLE 11 (Continued)

\begin{tabular}{|c|c|c|c|c|c|c|c|c|}
\hline $\mathrm{J}$ & $P(J)$ & $0-C$ & $P(J)$ & $0-C$ & $R(J)$ & $0-C$ & $R(J)$ & $0-c$ \\
\hline 29 & 3198.19 & -0.04 & 3196.86 & -0.03 & 3335.96 & -0.01 & & \\
\hline 30 & 3195.61 & -0.02 & 3194.20 & -0.01 & 3337.98 & +0.02 & & \\
\hline 31 & 3193.01 & -0.02 & 3191.50 & -0.01 & & & & \\
\hline 32 & 3190.40 & -0.01 & 3188.78 & -0.01 & & & & \\
\hline 33 & 3187.77 & -0.01 & 3186.07 & 0.00 & & & & \\
\hline 34 & 3185.13 & 0.00 & 3183.34 & +0.01 & & & & - \\
\hline 35 & 3182.50 & +0.03 & 3180.60 & +0.03 & & & & \\
\hline 36 & 3179.82 & +0.03 & & & . & & & \\
\hline
\end{tabular}

CONSTANTS DERIVED: band center $=3269.560 \pm 0.010$

$$
\begin{array}{rl}
\left(B^{\prime}-B^{\prime \prime}\right) & \text { ave. } \\
\left(D^{\prime}-D^{\prime \prime}\right) & -4.11 \times 10^{-3} \\
q^{\prime}=6.00 \times 10^{-3} & 0.88 \times 10^{-6} \\
& q_{5}=q^{\prime \prime}=4.60 \times 10^{-3}
\end{array}
$$

${ }^{a}$-doublets unresolved 
TABLE 12

ROTATIONAL ANALYSIS OF THE BAND $v_{2}+2 v_{4}^{2}+v_{5}{ }^{1}-v_{4}^{1}$

\begin{tabular}{|c|c|c|c|c|c|c|}
\hline \multicolumn{7}{|c|}{ Q-branch at 3269.70 (All units $\mathrm{cm}^{-1}$ ) } \\
\hline$J$ & $P(J)$ & $0-C$ & $P(J)$ & $0-C$ & $R(J)^{a}$ & $\begin{array}{l}0-C \\
\text { ave. }\end{array}$ \\
\hline 1 & & & & & 3274.50 & +0.07 \\
\hline 2 & & 3265.05 & $+0.03^{a}$ & & & \\
\hline 3 & & 3262.65 & 0.00 & & 3279.11 & +0.03 \\
\hline 4 & & 3260.27 & 0.00 & & 3281.49 & +0.10 \\
\hline 5 & . & 3257.88 & 0.00 & & 3283.71 & +0.02 \\
\hline 6 & & & . & & 3285.88 & -0.10 \\
\hline 7 & & & & & 3288.24 & -0.02 \\
\hline 8 & & & & & 3290.51 & -0.02 \\
\hline 9 & & & & & 3292.78 & -0.01 \\
\hline 10 & & & & & 3295.08 & +0.03 \\
\hline 11 & 3243.47 & -0.01 & & & 3297.29 & 0.00 \\
\hline 12 & 3241.02 & -0.04 & 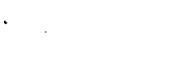 & & 3299.51 & 0.00 \\
\hline 13 & 3238.59 & -0.03 & 3238.24 & -0.08 & 3301.71 & -0.02 \\
\hline 14 & 3236.18 & 0.00 & 3235.83 & -0.01 & 3303.94 & 0.00 \\
\hline 15 & 3233.72 & -0.01 & 3233.28 & -0.06 & 3306.17 & +0.04 \\
\hline 16 & 3231.25 & -0.03 & 3230.81 & -0.03 & 3308.31 & 0.00 \\
\hline 17 & 3228.81 & 0.00 & 3228.31 & -0.02 & 3310.49 & +0.01 \\
\hline
\end{tabular}


TABLE 12 (Continued)

\begin{tabular}{|c|c|c|c|c|c|c|}
\hline $\mathbf{J}$ & $P(J)$ & $0-c$ & $P(J)$ & $0-c$ & $R(J)$ & $\begin{array}{l}0-c \\
\text { ave. }\end{array}$ \\
\hline 18 & 3226.35 & +0.01 & 3225.79 & -0.02 & 3312.59 & -0.04 \\
\hline 19 & 3223.86 & -0.01 & 3223.28 & 0.00 & 3314.74 & -0.03 \\
\hline 20 & 3221.40 & +0.02 & 3220.73 & -0.01 & 3316.87 & -0.02 \\
\hline 21 & 3218.87 & -0.01 & 3218.20 & +0.01 & 3318.97 & -0.03 \\
\hline 22 & 3216.38 & 0.00 & 3215.64 & +0.01 & & \\
\hline 23 & 3213.88 & +0.01 & 3213.05 & -0.01 & 3323.20 & +0.02 \\
\hline 24 & 3211.36 & +0.01 & 3210.50 & +0.02 & 3325.27 & +0.02 \\
\hline 25 & 3208.84 & +0.02 & 3207.90 & +0.01 & 3327.31 & +0.01 \\
\hline 26 & 3206.27 & -0.01 & 3205.30 & +0.01 & & \\
\hline 27 & 3203.70 & -0.03 & 3202.70 & +0.03 & & \\
\hline 28 & 3201.17 & 0.00 & 3200.07 & +0.02 & & \\
\hline 29 & 3198.59 & 0.00 & 3197.43 & +0.01 & & \\
\hline 30 & 3196.09 & +0.08 & 3194.82 & +0.05 & & \\
\hline 31 & 3193.47 & +0.05 & 3292.11 & 0.00 & & \\
\hline 32 & 3190.80 & -0.01 & 3189.44 & 0.00 & & \\
\hline 33 & 3188.17 & -0.02 & 3186.76 & 0.00 & & \\
\hline 34 & 3185.54 & -0.02 & 3184.06 & -0.01 & & \\
\hline 35 & $\cdots$ & & 3181.35 & -0.01 & & \\
\hline 36 & 3180.22 & -0.03 & 3178.62 & -0.02 & & \\
\hline 37 & & & 3175.90 & 0.00 & & \\
\hline 38 & & & 3173.15 & -0.01 & & \\
\hline
\end{tabular}


TABLE 12 (Continued)

CONSTANTS DERIVED: band center $=3269.74 \pm 0.01$

$$
\begin{aligned}
& \left(B^{\prime}-B^{*}\right)_{\text {ave. }}=-4.34 \times 10^{-3} \\
& \left(D^{\prime}-D^{\prime \prime}\right)=0.61 \times 10^{-6} \\
& q^{\prime}=4.36 \times 10^{-3} \quad q_{4}=q^{\prime \prime}=5.49 \times 10^{-3}
\end{aligned}
$$

${ }_{\text {a-doublets unresolved }}$ 
ROTATIONAL ANALYSis OF THE BAND $v_{2}+v_{4}{ }^{1}+2 v_{5}{ }^{2}-v_{5}{ }^{1}$

\begin{tabular}{|c|c|c|c|c|c|c|c|c|}
\hline $\mathrm{J}$ & $P(J)$ & $0-c$ & $\mathrm{P}(\mathrm{J})$ & $0-C$ & $R(J)$ & $0-C$ & $R(J)$ & $0-C$ \\
\hline 1 & & & & & 3291.08 & +0.04 & & \\
\hline 2 & & & 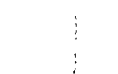 & & 3293.40 & +0.05 & & \\
\hline 3 & 3279.27 & -0.02 & & & & & & \\
\hline 4 & 3276.88 & -0.02 & & & & & & \\
\hline 5 & 3274.50 & -0.01 & & & . & & & \\
\hline 6 & 3272.09 & -0.01 & & & 3302.56 & +0.05 & & \\
\hline 7 & 3269.70 & +0.01 & & & . & & & \\
\hline 8 & 3267.25 & -0.01 & & & 3307.05 & +0.03 & & \\
\hline 9 & 3264.86 & +0.04 & & & 3309.28 & +0.02 & 3309.61 & +0.01 \\
\hline 10 & 3262.37 & 0.00 & & & 3311.51 & +0.03 & 3311.86 & -0.03 \\
\hline 11 & 3259.90 & -0.01 & & & 3313.69 & -0.01 & 3314.15 & -0.01 \\
\hline
\end{tabular}


TABLE 13 (Continued)

\begin{tabular}{|c|c|c|c|c|c|c|c|c|}
\hline $\mathrm{J}$ & $P(J)$ & $0-C$ & $P(J)$ & $0-C$ & $R(J)$ & $0-c$ & $\mathrm{R}(\mathrm{J})$ & $0-c$ \\
\hline 12 & 3257.42 & -0.01 & & & 3315.91 & +0.01 & 3316.44 & 0.00 \\
\hline 13 & 3254.93 & -0.02 & & & 3318.07 & -0.02 & 3318.68 & -0.02 \\
\hline 14 & 3252.43 & -0.02 & & & 3320.25 & -0.02 & & \\
\hline 15 & & & & & 3322.39 & -0.04 & 3323.20 & 0.00 \\
\hline 16 & 3247.39 & -0.03 & 3247.79 & 0.00 & 3324.60 & +0.02 & 3325.45 & +0.01 \\
\hline 17 & 3244.87 & -0.02 & 3245.31 & -0.01 & & . & 3327.66 & -0.01 \\
\hline 18 & 3242.32 & -0.02 & 3242.84 & -0.01 & 3328.87 & +0.02 & 3329.86 & -0.04 \\
\hline 19 & 3239.74 & -0.04 & 3240.37 & +0.01 & 3330.99 & +0.03 & 3332.12 & +0.01 \\
\hline 20 & 3237.19 & -0.01 & 3237.86 & -0.01 & 3333.07 & 0.00 & 3334.33 & +0.02 \\
\hline 21 & 3234.62 & 0.00 & 3235.37 & -0.01 & 3335.14 & 0.00 & 3336.53 & +0.02 \\
\hline 22 & 3232.02 & 0.00 & 3232.86 & -0.01 & 3337.18 & -0.03 & 3338.73 & +0.04 \\
\hline
\end{tabular}


TABLE 13 (Continued)

\begin{tabular}{|c|c|c|c|c|c|c|c|c|}
\hline $\mathrm{J}$ & $P(J)$ & $0-c$ & $P(J)$ & $\mathrm{O}-\mathrm{C}$ & $\mathrm{R}(\mathrm{J})$ & $0-C$ & $R(J)$ & $0-c$ \\
\hline 23 & 3229.41 & +0.01 & 3230.39 & +0.03 & 3339.28 & +0.01 & 3340.85 & -0.02 \\
\hline 24 & 3226.80 & +0.02 & & & 3341.33 & +0.02 & & \\
\hline 25 & & & & & & & 3345.22 & +0.04 \\
\hline 26 & 3221.51 & +0.03 & & & 3345.34 & +0.01 & 3347.35 & +0.02 \\
\hline 27 & 3218.87 & +0.06 & & & 3347.35 & 0.00 & 3349.46 & 0.00 \\
\hline 28 & 3216.19 & +0.07 & . & & 3349.29 & -0.04 & 3351.57 & -0.01 \\
\hline 29 & & & .' & & 3351.29 & 0.00 & 3353.67 & -0.02 \\
\hline 30 & 3210.70 & 0.00 & $\therefore$ & . & 3353.20 & -0.03 & & \\
\hline 31 & & & $\because$ & & 3355.16 & 0.00 & & \\
\hline 32 & 3205.20 & -0.02 & 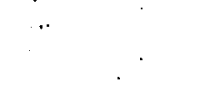 & & 3357.05 & -0.03 & & \\
\hline 33 & 3202.38 & -0.07 & : & & 3359.00 & +0.03 & & \\
\hline
\end{tabular}


TABLE 13 (Continued)

\begin{tabular}{|c|c|c|c|c|c|c|c|c|c|}
\hline $\mathrm{J}$ & $P(J)$ & $0-C$ & $\because$ & $\mathrm{P}(\mathrm{J})$ & O-C. & $R(J)$ & $0-C$ & $R(J)$ & $0-c$ \\
\hline 34 & & & & & & 3360.84 & 0.00 & 3361.94 & -0.06 \\
\hline 35 & & & & & 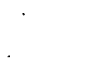 & & & & \\
\hline 36 & & & & & & & 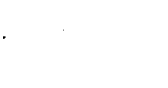 & & \\
\hline 37 & & & & $\ldots$ & & & & & \\
\hline 38 & & & & & & 3368.18 & +0.03 & & \\
\hline
\end{tabular}

CONSTANTS DERIVED: band center $=3286.36 \pm 0.01$

$$
\begin{array}{cc}
\left(B^{\prime}-B^{\prime \prime}\right)_{\text {ave. }} & =-4.38 \times 10^{-3} \\
\left(D^{\prime}-D^{\prime \prime}\right) & =0.50 \times 10^{-6} \\
q^{\prime}=6.88 \times 10^{-3} & q_{5}=q^{\prime \prime}=4.60 \times 10^{-3}
\end{array}
$$


although Fig. 12 shows that the formula above is a good approximation; because of the apparent deviation at high J-values, however, the calculated values in Tables $10-13$ do not involve the constraint imposed by the $\Delta y$ formula.

Some blended lines are listed in the tables; these were not included in determining the constants. Lines for which no values are given in the tables were not observed, usually due to blending. The values of $q_{5}$ determined agree extremely well with those of previous investigators (12). Those for $q_{4}$ are not quite as accurate, due primarily to the fact that our data resolved the doubling on only one branch for the two bands having lower state 00010; this leads to greater inaccuracy in the determination of $q^{\prime}$ and $q^{\prime \prime}$ for those bands than arises when doubling is measured in both branches. 
Figure 12

Graphica1 Determination of Rotational $\ell$-type Doubling Constants 


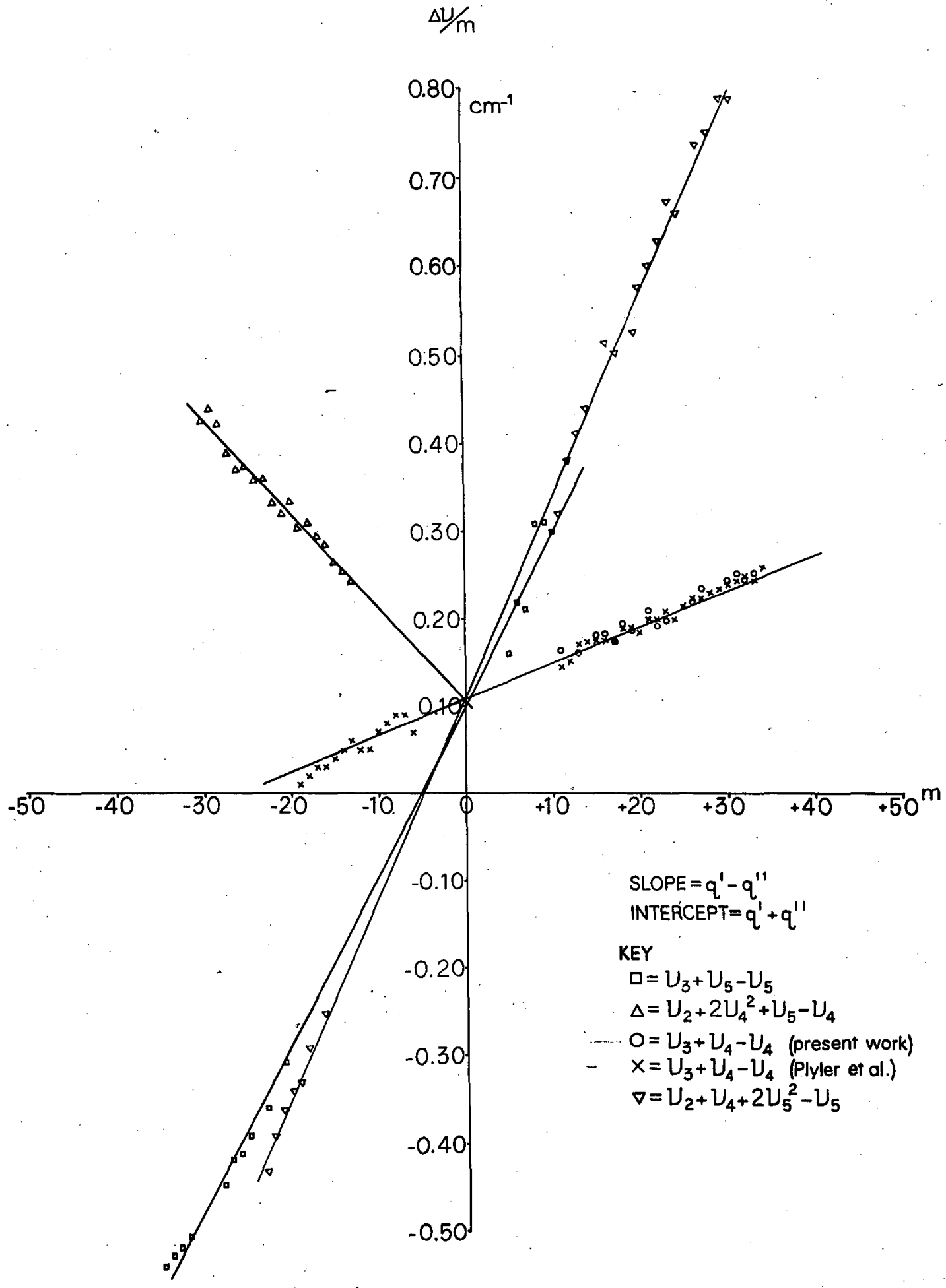




\section{CHAPTER V}

\section{LOOMIS-WOOD DIAGRAMS FOR POLYATOMIC}

\section{INFRARED SPECTRA}

In Chapters II and IV the regions of the two active fundamentals, three and fourteen microns, were investigated. Both of the fundamentals are badly overlapped at normal temperatures, due to the presence of transitions from the low-lying vibrational states associated with the two bending modes. Since many of the upper states involved in these difference bands do not yield infrared-active transitions from the ground state, there is considerable interest in the analysis of these overlapping bands. It has been found that the diagram method originally described by Loomis and Wood $(1,17)$ is very helpful in sorting out the overlapping bands.

In the spectra of linear molecules usually a series of evenlyspaced lines stand out clearly as belonging to the same band in an overlapped region. By forming first and second differences for the band and keeping the second difference constant, the expected positions of additional lines belonging to this band may be calculated. If the series of lines is indeed a band, then further lines will be found at each of the calculated positions, with a regular intensity pattern evident. These lines may then be numbered by assignment of an arbi- 
trary running number $\mathrm{m}$; they form the abscissa axis of our diagram. For each line of the calculated band, differences are formed with all the neighboring spectral lines; these differences are plotted versus the running number $\mathrm{m}$, as shown on the accompanying figures.

Fig. 13 is a diagram of the spectra at 13.7 microns. $P$ and $R$ branch lines of the $v_{5}$ fundamental were used as running numbers since their intensity made this an easy choice. Differences between each line of $v_{5}$ and its neighbors are plotted above or below the point on the abscissa representing that $v_{5}$ line. The result is a series of smooth curves, each consisting of a single band. The curvature of the plots is due to the term $\left(B_{v}^{*}-B_{v}^{*}\right) J^{2}$, and is very noticeable for most of the bands in this figure. Band $B$ does not exhibit such curvature, indicating that $B^{*}$ is very nearly equal to $B^{\prime \prime}$ for this band; the negative slope of this band shows that for it the term $\left(B_{v}^{\prime}+B_{v}^{N}\right)$ is much less than $B^{\prime}+B^{\prime \prime}$ of the band $\left(v_{5}\right)$ used as abscissa -- and points out that band $B$ is due to the isotopic species involving carbon-13. This was a surprising observation, since a natural, unenriched sample of acetylene had been used. Fig. 13 shows more clearly the effect of $\mathrm{B}^{\prime \prime}+\mathrm{B}^{\prime \prime}$ and $\mathrm{B}^{\ell}-\mathrm{B}^{\prime \prime}$ values than most similar diagrams do. This is because the band chosen as abscissa has $\mathrm{B}^{l}$ very nearly equal to $B^{\prime \prime}$ and because the band centers of the overlapping bands are all near the band center of $v_{5}$. In general it is not quite so easy to pick out isotopic transitions, or to locate band centers -- which are at the apex of each parabolic curve in Fig. 13. Fig. 14 demonstrates this quite we11. 
Figure 13

Loomis-Wood Diagram of the Spectrum at 13.7 Microns 


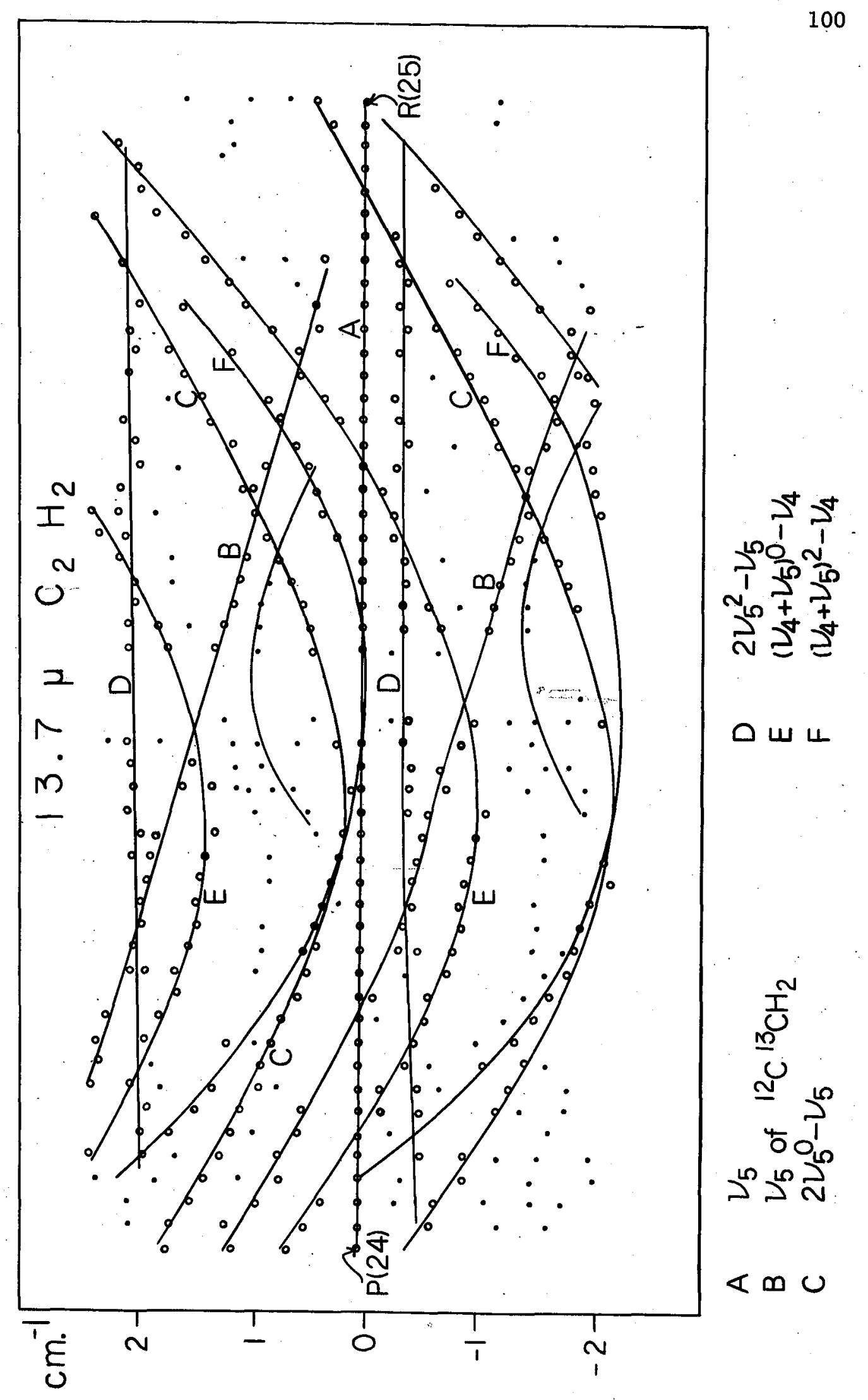


Figure 14

Loomis-Wood Diagram of the Spectrum at 3 Microns 


\section{LOOMIS-WOOD DIAGRAM OF $3 \mu \mathrm{C}_{2} \mathrm{H}_{2}$ SPECTRA}

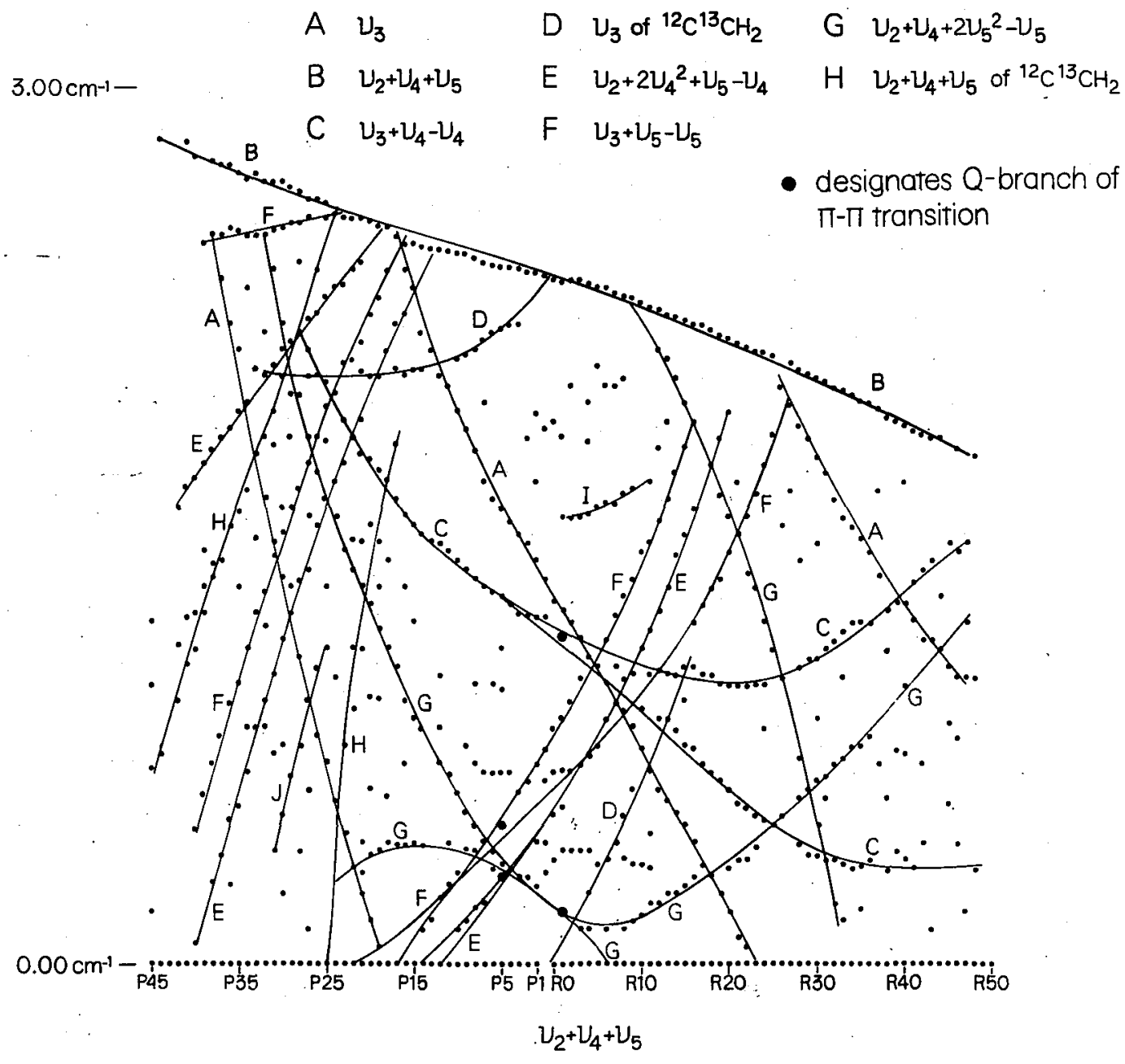


Fig. 14 shows a diagram involving approximately 700 lines measured in the three micron region. As in Fig. 13, the dots which do not fall on drawn curves generally correspond to spectral lines of very weak intensity which were not identified. In Fig. 14, m-values of the $P$ and $R$ branch lines of the strong combination band $v_{2}+v_{4}+v_{5}$ were used as running numbers. Since $\mathrm{B}^{\prime} \neq \mathrm{B}^{\prime \prime}$ for this band, and since the band centers of the overlapping bands are not close together, the effect of $B^{\prime}+B^{\prime \prime}$ and $B^{i}-B^{\prime \prime}$ terms seen in Fig. 13 is not obvious here. Nevertheless, the bands do fall on smooth curves, and the main job of analysis can be done as well as in Fig. 13. The most striking feature of Fig. 14 is the presence of $\Pi-\Pi$ bands $C, E, F$ and $G$. These transitions all exhibit resolved rotational $\ell$-type doubling which manifests itself as discernible splitting on the P-branch side, R-branch side, or both. Band $\mathrm{C}$ has been discussed in the past by Plyler et al. (12); the others are previously unreported. Note that band G exhibits extremely large doubling in both $P$ and $R$ branches; this is due to the large difference $q^{\prime}-q^{\prime \prime}$ between the rotational $\ell$-type doubling constants of the upper and lower states.

It has been found that this diagram method has been helpful in analyzing overlapping bands of $\mathrm{C}_{2} \mathrm{D}_{2}$ and of $\mathrm{CO}_{2}$, in addition to those cases shown here. Moreover, it can also readily by used for analysis of parallel bands of symmetric top molecules; it was found, using data on $\mathrm{ND}_{3}$, that the $\mathrm{K}$-subbands could be treated exactly as overlapping bands of a linear molecule. The present interest in "hot" bands of 
symmetric tops and the problem of vibrational b-type doubling in such molecules led to consideration of this; it appears that the diagram techniques discussed here might aid in the initial analysis of such spectra. 
CHAPTER VI

BANDS IN THE VISIBLE AND PHOTOGRAPHIC INFRARED

The present chapter is concerned with the analysis of nine vibration rotation bands of ${ }^{12} \mathrm{C}_{2} \mathrm{H}_{2}$ which occur in the visible and near infrared regions of the spectrum. A11 of these bands have been observed before; however, band centers and B-values have not been determined for them using modern techniques. Further, there has been a lack of knowledge of numerical values of the vibrational constants involved in the expressions for the upper states of the transitions considered here. Because of this, the assignment of two of these bands has been viewed as very uncertain (1).

The data presented in this chapter were obtained with a tenmeter spectrograph ${ }^{a}$ in a Czerny-Turner mounting. For a1l of the bands presented here, a path length of forty meters was used, with pressures from $100 \mathrm{~mm}$. to $1 \mathrm{~atm}$. Iron arc emission standards were used for calibration; a third-order dispersion curve was determined for each plate by means of a least-squares fit of the iron lines.

Because fewer lines are observable in vibration rotation bands of acetylene occurring in the photographic region than are encountered

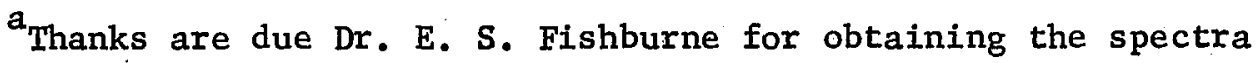
used in this study. 
in the infrared bands, calculation of rotational constants $B$ and $D$ is not as accurate. If one has only fifteen or twenty lines, calculation of $B$ and $D$ by means of least-squares fits to combination differences is apt to be very unsatisfactory; and, in cases such as those encountered in the present work at 8640 and $6850 \AA$, where only three or four lines are measurable on the P-branch side, combination differences are complete1y useless. On the other hand, a fourth-order fit to $\nu(m)$, given by the first five terms of Eq. (17) uses every data point; its disadvantage, compared to the combination differences, is that it fits the data to five constants $\left(v_{0}, B^{\prime}, B^{\prime \prime}, D^{\prime}, D^{\prime \prime}\right)$ and not two or three, with a high uncertainty resulting for each constant. Because of this problem, a third method, described below, has been employed to determine rotational constants from the observational data.

With the single exception of the $\Pi$ - $\Pi$ transition at $12618 \mathrm{~cm}^{-1}$, al1 of the bands analyzed here are transitions from the ground state. The $\mathrm{B}_{0}, \mathrm{D}_{0}$ values for the ground state are very well known (see Table 9 of Chapter IV). If the expression for $v(m)$ is rearranged as

$$
\begin{aligned}
v(m)-B^{\prime \prime}\left(m-m^{2}\right)-D^{\prime \prime}\left(-2 m^{3}+m^{4}\right)=v_{0} & +B^{2}\left(m+m^{2}\right) \\
& +D^{\prime}\left(2 m^{3}+m^{4}\right)
\end{aligned}
$$

where the fact that $D \ll B$ for acetylene allows the terms $\mathrm{Dm}^{2}$ to be dropped (compare with Eq. (1.7)), then the $B^{\prime \prime}=B_{0}$ and $D^{\prime \prime}=D_{0}$ terms can be calculated and the left side of Eq. (30) can be fitted to only three 
unknowns. This has, in fact, been done with the data of this chapter. A multiple regression procedure developed by Mr. J. Luce of our laboratory has been used to fit the data to the expression in Eq. (30). This program employs an iterative matrix inversion and double precision arithmetic and exceeds the accuracy of the usual least squares fits. With it the constants given in Table 15 were determined from the data tabulated in Table 14. The uncertainty listed with each band center, $B$, or $D$ value is the standard deviation; Table 15 shows that a high level of accuracy and small confidence interval was obtained with relatively. few data points.

Assignment of the bands at 11570.73 and $11586.43 \mathrm{~cm}^{-1}$ is discussed below. The upper states of these bands had been assigned by Funke (18) as $2 a, 2,2 s$ and $1 a, 3,4 s$ in the notation of his day, where the quantum numbers are $\mathrm{V}_{\mathrm{C}-\mathrm{H}}, \mathrm{V}_{\mathrm{C}-\mathrm{C}}$, and $\mathrm{V}_{\text {bend }}$ and the letters indicate asymmetric or symmetric. Herzberg (1), however, lists them as $\left[1212^{0} 0^{0}\right.$ ? $]$ and $\left[1303^{1} 1^{1}\right.$ ?]. The two notations refer to the same state in the first case, although Funke's is somewhat ambiguous; however, Funke's assignment of the latter state would be $0314^{0} 0^{0}$ in our notation. The data presented in this chapter agree perfectly with Herzberg's assignment.

The vibrational constants required for the calculations pursued here are known reasonably well from Reference 3 and the previous chapters. Conspicuous by its absence from 1ists in these two places is $\mathrm{x}_{22}$. Since $\mathrm{x}_{22}$ is important in the photographic bands, it is calculated here. The calculation below uses the value of $(00100$ unperturbed $)$ 
TABLE 14

LINE POSITIONS OF SOME PHOTOGRAPHIC BANDS

ROTATIONAL ANALYSIS OF THE BAND $5 v_{3}$

(A11 units $\mathrm{cm}^{-1}$ )

\begin{tabular}{|c|c|c|c|c|}
\hline $\mathrm{J}$ & $P(J)$ & $\mathrm{O}-\mathrm{C}$ & $R(J)$ & $0-C$ \\
\hline 0 & & & & \\
\hline 1 & & & & \\
\hline 2 & & & & \\
\hline 3 & 15592.89 & 0.00 & 15608.93 & 0.00 \\
\hline 4 & 15590.36 & -0.01 & 15610.96 & +0.01 \\
\hline 5 & 15587.74 & -0.01 & 15612.92 & +0.02 \\
\hline 6 & 15585.06 & 0.00 & 15614.80 & +0.01 \\
\hline 7 & 15582.32 & $\therefore+0.01$ & $\therefore, \cdots$ & - \\
\hline $8^{*}$ & 15579.54 & $\ldots+0.04$ & 15618.35 & -0.02 \\
\hline 9 & 15576.64 &.+0.02 & 15620.05 & -0.01 \\
\hline 10 & 15573.67 & 0.00 & 15621.64 & -0.04 \\
\hline 11 & 15570.70 & +0.04 & 156.23 .23 & -0.01 \\
\hline 12 & 15567.58 & 0.00 & 15624.73 & 0.00 \\
\hline 13 & 15564.44 & +0.01 & 15626.16 & +0.01 \\
\hline 14 & 15561.23 & +0.01 & 15627.51 & 0.00 \\
\hline 15 & 15557.97 & +0.02 & 15628.80 & -0.01 \\
\hline 16 & & & 15630.02 & -0.01 \\
\hline 17 & & & 15631.17 & -0.02 \\
\hline
\end{tabular}


TABLE 14 (Continued)

\section{ROTATIONAL ANALYSIS OF THE BAND $5 v_{3}$ \\ (All units $\mathrm{cm}^{-1}$ )}

\begin{tabular}{|c|c|c|c|c|}
\hline $\mathbf{J}$ & $P(J)$ & $0-C$ & $R(J)$ & $0-C$ \\
\hline 18 & 15547.73 & -0.01 & & \\
\hline 19 & 15544.21 & +0.01 & & \\
\hline 20 & & & 15634.28 & 0.00 \\
\hline 21 & 15537.00 & +0.06 & & \\
\hline 22 & & & & \\
\hline 23 & 15529.44 & +0.02 & 15636.75 & -0.02 \\
\hline 24 & & & & \\
\hline 25 & & & 15638.10 & -0.01 \\
\hline
\end{tabular}

ROTATIONAL ANALYSIS OF THE BAND $v_{1}+3 v_{2}+3 v_{4}^{1}+v_{5}^{1}$

(A11 units $\mathrm{cm}^{-1}$ )

\begin{tabular}{llllll}
\hline $\mathrm{J}$ & $\mathrm{P}(\mathrm{J})$ & $\mathrm{O}-\mathrm{C}$ & $\mathrm{R}(\mathrm{J})$ & $\mathrm{O}$ \\
\hline
\end{tabular}

0

1 .

11591.00

$-0.03$

2

3

11579.32

$+0.05$

11595.49

0.00

4

11597.69

$+0.02$

5

11574.37

$+0.05$

11599.85

$+0.04$

6

11601.90

$-0.01$ 
TABLE 14 (Continued)

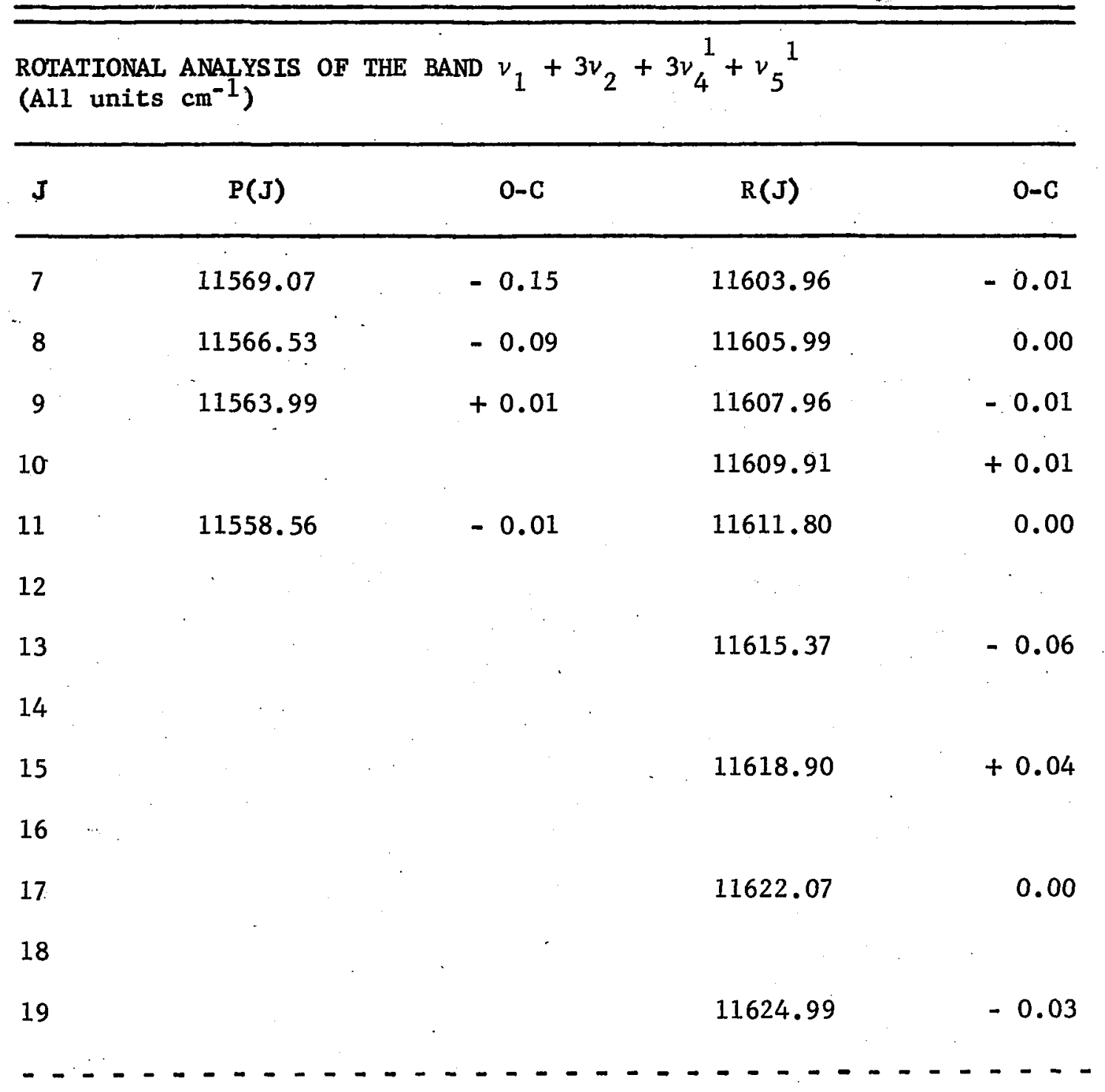


TABLE 14 (Continued)

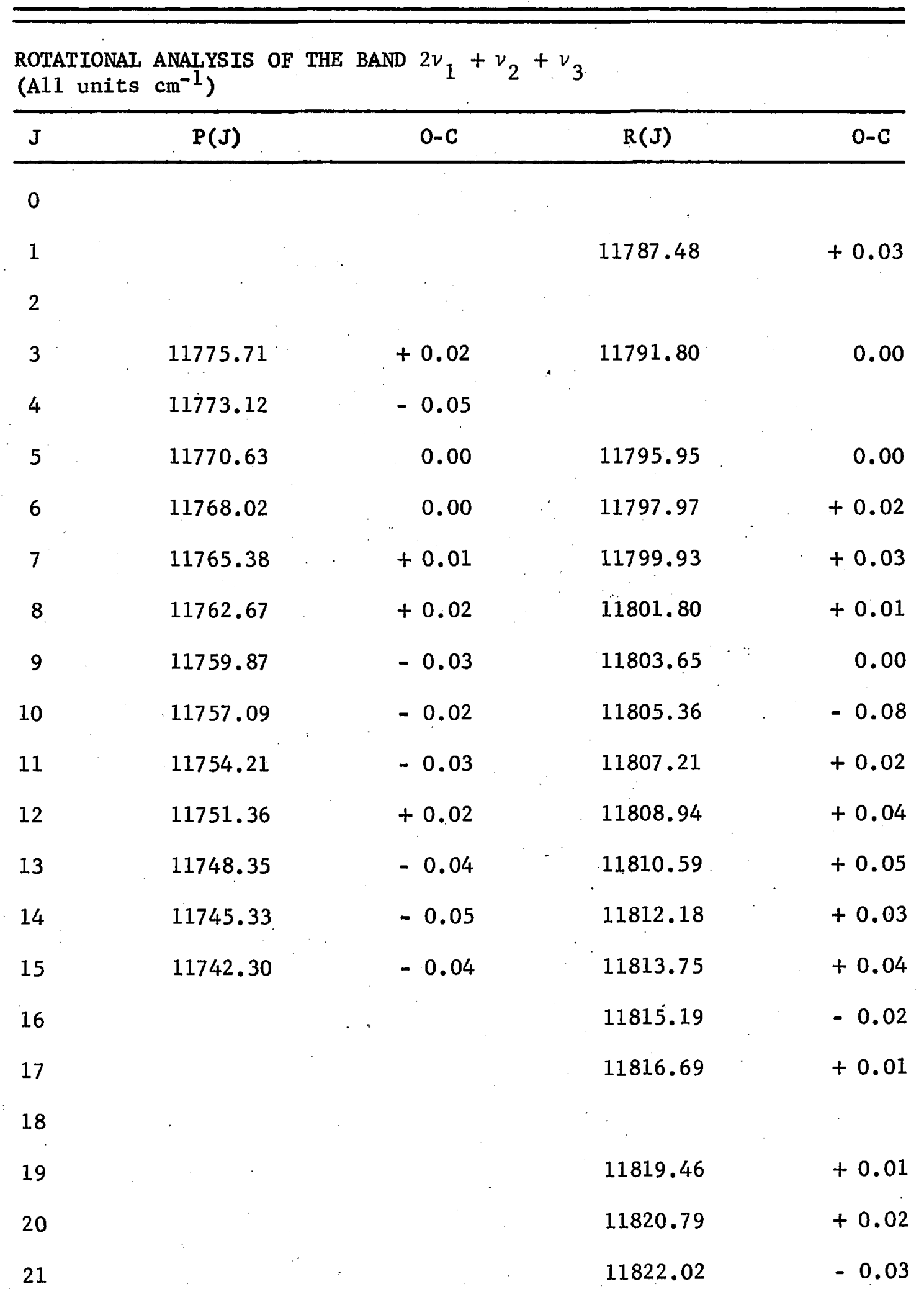


TABLE 14 (Continued)

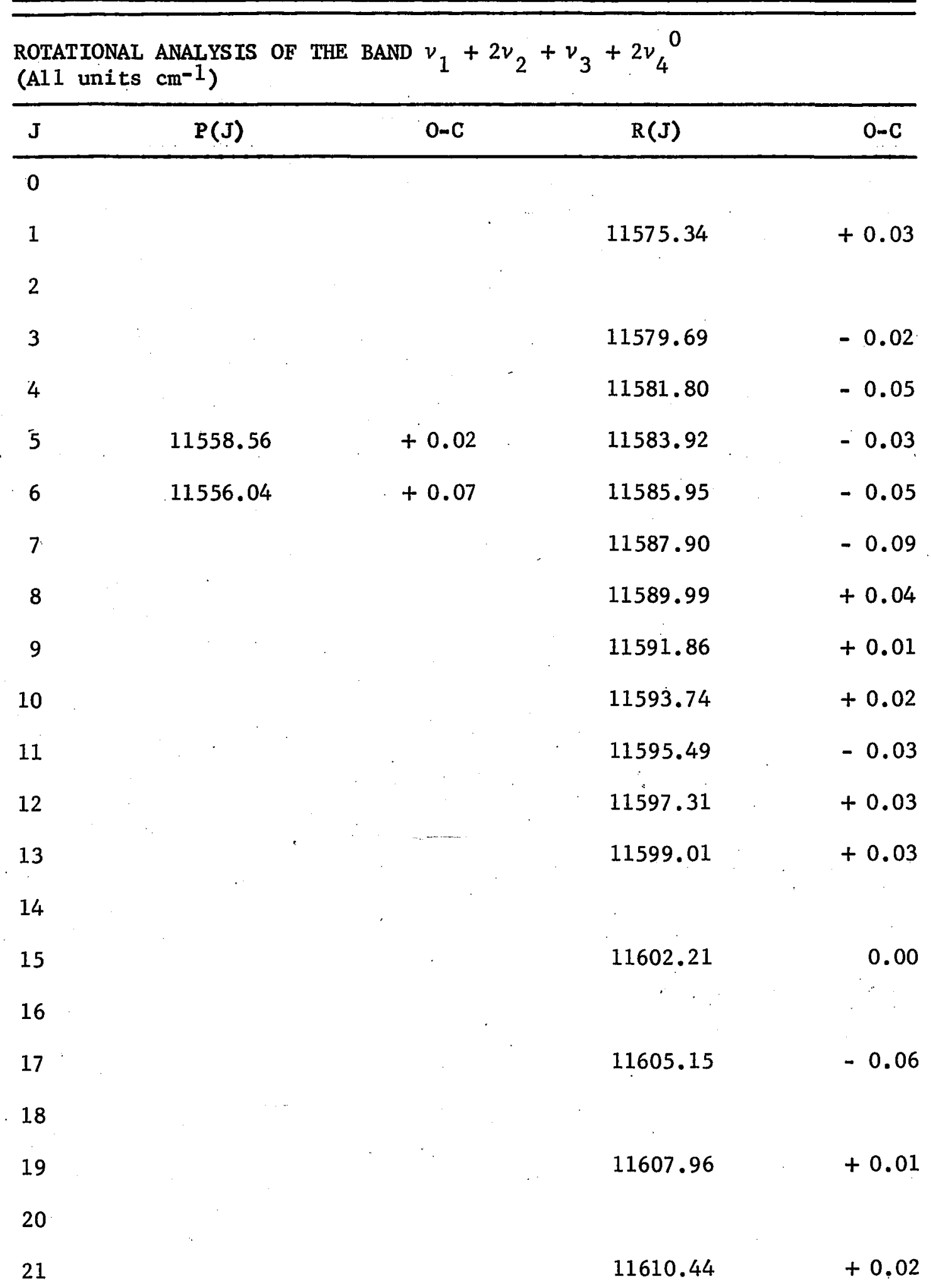


TABLE 14 (Continued) ROTATIONAL ANALYSIS OF THE BAND $v_{1}+v_{2}+3 v_{3}$
(A11 units $\mathrm{cm}^{-1}$ )

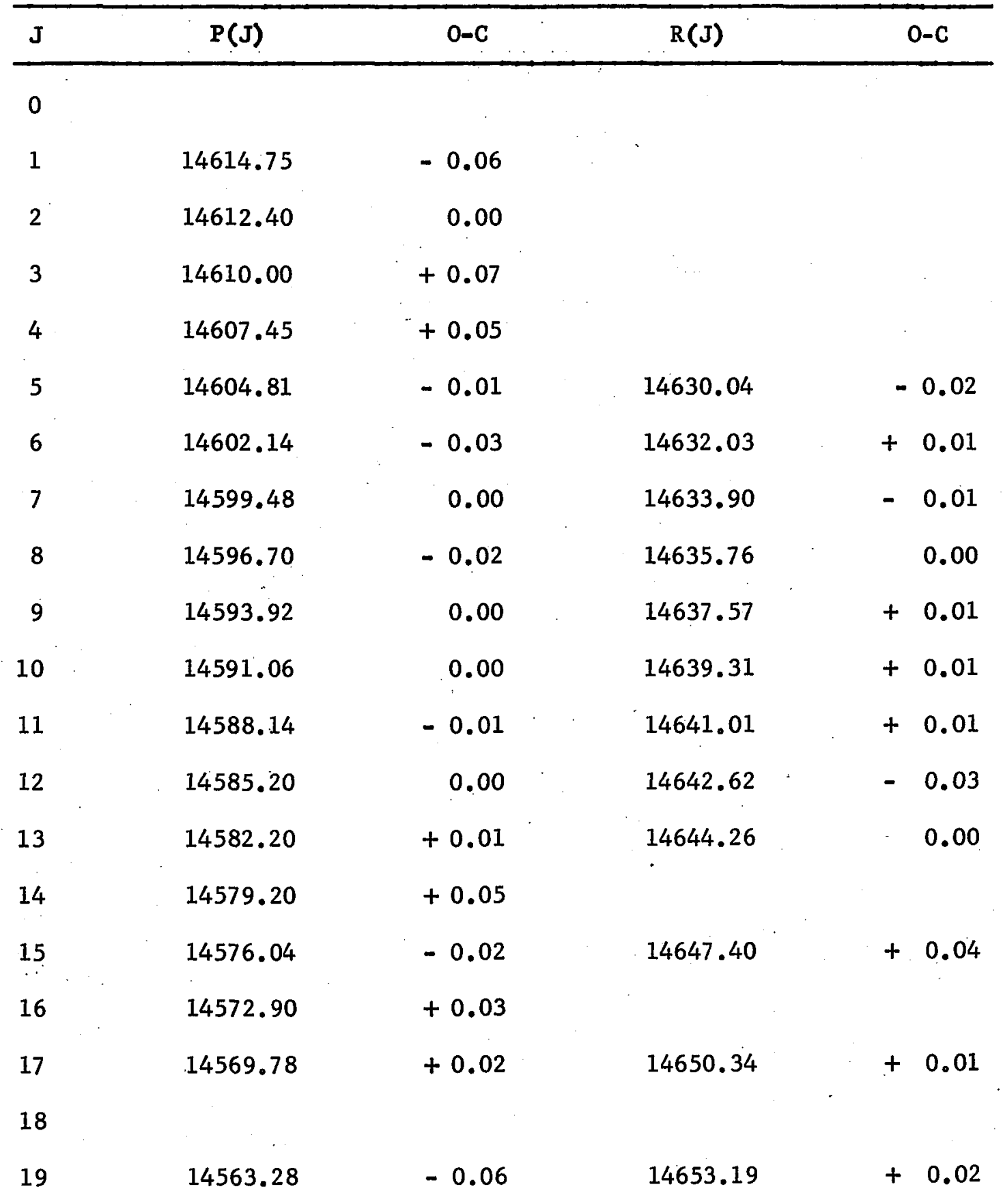


TABLE 14 (Continued)

\begin{tabular}{|c|c|c|c|c|}
\hline $\mathbf{J}$ & $P(J)$ & $0-c$ & $R(J)$ & $0-C$ \\
\hline 0 & & & & \\
\hline 1 & - & & & \\
\hline 2 & & & & \\
\hline 3 & & & 14605.99 & +0.03 \\
\hline 4 & 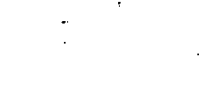 & & 14608.08 & +0.03 \\
\hline 5 & 14584.69 & -0.09 & 14610.00 & -0.08 \\
\hline 6 & & & 14612.15 & +0.08 \\
\hline 7 & 14579.42 & -0.08 & 14614.01 & +0.01 \\
\hline 8 & 14576.84 & +0.07 & 14615.96 & +0.08 \\
\hline 9 & 14574.00 & 0.00 & 14617.73 & +0.02 \\
\hline 10 & & & & \\
\hline 11 & 14568.29 & -0.02 & 14621.26 & +0.03 \\
\hline 12 & & & & \\
\hline 13 & - & & 14624.58 & +0.03 \\
\hline 14 & & & 14626.04 & -0.10 \\
\hline 15 & & & 14627.67 & -0.02 \\
\hline 16 & & & 14629.10 & -0.10 \\
\hline 17 & & & 14630.78 & +0.12 \\
\hline
\end{tabular}


TABLE 14 (Continued)

ROTATIONAL ANALYSIS OF THE BAND $v_{2}+3 v_{3}$
(A11 units $\mathrm{cm}^{-1}$ )

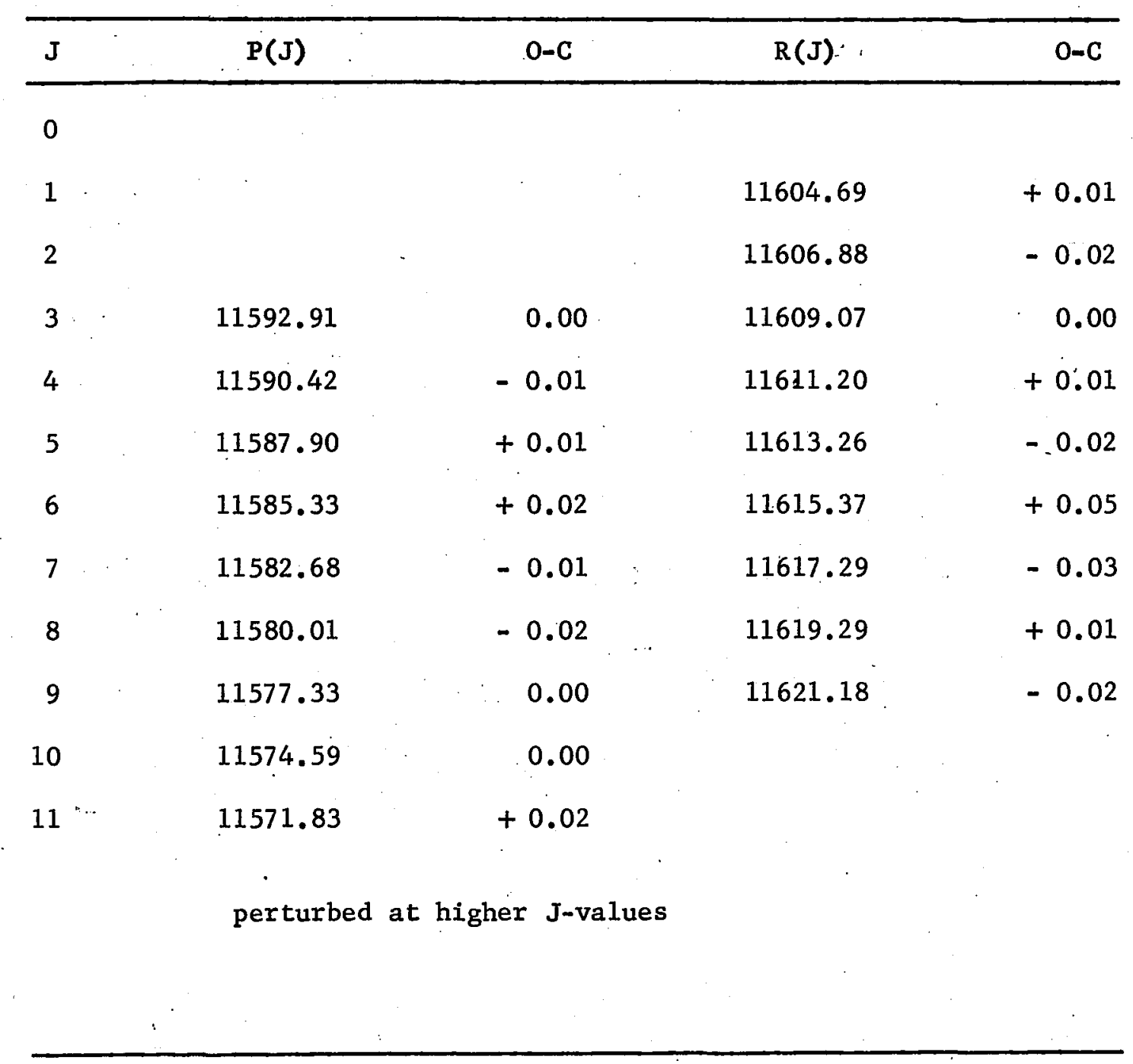


TABLE 15

ROTATION CONSTANTS DERIVED FROM THE PHOTOGRAPHIC BANDS (A11 values are vac. $\mathrm{cm}^{-1}$; uncertainties are standard deviations)

\begin{tabular}{ccccc}
\hline $\begin{array}{l}\text { UPPER } \\
\text { STATE }\end{array}$ & $\begin{array}{l}\text { LOWER } \\
\text { STATE }\end{array}$ & BAND CENTER & $B^{2}$ & $D^{\prime} \times 10^{6}$ \\
\hline $1212^{0} 0$ & 00000 & $11570.729 \pm 0.02$ & $1.15533 \pm 0.0002$ & $6.4 \pm 0.4$ \\
$1303^{1} 1$ & 00000 & $11586.431 \pm 0.02$ & $1.15922 \pm 0.0003$ & $7.9 \pm 0.8$ \\
01300 & 00000 & $11600.110 \pm 0.01$ & $1.15375 \pm 0.0006$ & $5.2 \pm 5.0$ \\
21100 & 00000 & $11782.894 \pm 0.01$ & $1.15125 \pm 0.0001$ & $4.5 \pm 3.0$ \\
10310 & 00010 & $126.18 .520 \pm 0.05$ & $1.15055 \pm 0.0011$ & $6.9 \pm 4.5$ \\
10300 & 00000 & $12675.691 \pm 0.01$ & $1.15132 \pm 0.0001$ & $1.0 \pm 0.2$ \\
$0232^{0} 0$ & 00000 & $14597.082 \pm 0.04$ & $1.14990 \pm 0.0007$ & $1.8 \pm 2.1$ \\
11300 & 00000 & $14617.165 \pm 0.01$ & $1.14729 \pm 0.0002$ & $5.9 \pm 0.4$ \\
00500 & 00000 & $15600.176 \pm 0.008$ & $1.14345 \pm 0.00006$ & $1.30 \pm 0.10$ \\
\hline
\end{tabular}


from Reference 2 and the level 03100. This level was chosen since it appears to be free of resonance; unlike many of the upper levels of photographic bands, it cannot exhibit Darling-Dennison resonance, and the Fermi resonance with $0401^{1} 1^{1}$ is apparently very weak, since no neighboring band is in evidence which could be assigned as 04011 .

$$
\begin{aligned}
01000 & =\omega_{2}+x_{22}=1973.99 \\
03000 & =03100-\left(00100_{\text {unperturbed }}\right)-3 x_{23} \\
& =9151.70-3288.66+8.46=5871.30 \\
& =3 \omega_{2}+9 x_{22}
\end{aligned}
$$

Eqs. (31) and (32) yield $\omega_{2}=1990.88$ and $x_{22}=-16.89 \mathrm{~cm}^{-1}$. These values are considered superior to those presented elsewhere. With them, $1303^{1} 1^{1}$ is calculated as

$$
\begin{aligned}
1303^{1} 1^{1}= & \left(0003^{1} 1^{1}\right)+(03000)+(10000)+3 x_{12} \\
& +3 x_{14}+x_{15}+3 x_{25}+9 x_{24} \\
= & 2560.59+5871.30+3372.83-39.27-49.62 \\
& -10.85-4.14-114.30=11586.54 \mathrm{~cm}^{-1},
\end{aligned}
$$

compared to the value of 11586.43 observed. 
Similarly,

$$
\begin{aligned}
1212^{0} 0^{0}= & 11100+01000+0002^{2} 0^{0}-4 g_{44} \\
& +x_{12}+x_{23}+4 x_{24}+2 x_{14}+2 x_{34}+2 x_{22} \\
= & 8512.10+1973.99+1235.07+0.88 \\
& -1309-2.82-50.80-33.08-16.44 \\
& -33.78=11572.03 \mathrm{~cm}^{-1}
\end{aligned}
$$

compared to $11570.73 \mathrm{~cm}^{-1}$ observed. Since the value of $\mathrm{g}_{44}$ used above is relatively uncertain (see Chapter IV), this agreement is quite satisfactory.

Comparison of calculated and observed values of $B^{\prime}-B^{\prime \prime}$ for these bands is made below, using the data from Table 15 and the $\alpha$ 's from Reference 3:

Band

$$
\text { Obs. }\left(B^{\prime}-B^{\prime \prime}\right) \times 10^{3}
$$

Ca1c. $\times 10^{3}$

$0-C \times 10^{3}$

$1212^{0} 0^{0}-00000$

$$
21.27 \pm 0.20
$$

22.42

$-1.15 \pm 0.20$

$1303^{1} 1^{1}-00000$

$17.38 \pm 0.30$

17.58

$-0.20 \pm 0.30$

The agreement is very good. The calculated value for $1303^{1} 1^{1}$ was obtained as $B^{\prime \prime}-B^{\prime \prime}=\alpha_{1}+3 \alpha_{3}+\left(\alpha_{\text {effective }}\right) 0003^{1} 1^{1}$, where $\alpha_{\text {effective }}=B^{\prime}-B_{0}$ for the band $0003^{1} 1^{1}$. This was done so that the rotational effects of the off-diagonal elements due to vibrational and 
rotational l-type effects and shown in Matrix $V$ are completely included. This is an important point, since the rotational l-type doubling constants $q_{i}$ in Matrix $V$ make $\left(\alpha_{\text {effective }}\right) 0003^{1} 1^{1}$ differ from $3 \alpha_{4}+\alpha_{5}$ by $2.04 \times 10^{-3}$, a large amount compared to the 0-C differences above. The $\left(B^{\prime}-B^{\prime \prime}\right)$ values for the other six transitions studied are given below $\left(\mathrm{cm}^{-1}\right)$ :

$\begin{array}{lccc}\text { Transition } & \text { Obs. }\left(B^{\prime}-B^{\prime \prime}\right) \times 10^{3} & \text { Calc. } \times 10^{3} & 0-C \times 10^{3} \\ 01300-00000 & 22.85 \pm 0.60 & 23.10 & -0.25 \pm 0.60 \\ 21100-00000 & 25.35 \pm 0.10 & 25.54 & -0.19 \pm 0.10 \\ 10300-00000 & 25.38 \pm 0.10 & 23.62 & 1.76 \pm 0.10 \\ 02320-00000 & 26.70 \pm 0.70 & 26.80 & -0.10 \pm 0.70 \\ 11300-00000 & 29.31 \pm 0.20 & 29.92 & -0.61 \pm 0.20 \\ 00500-00000 & 33.15 \pm 0.06 & 28.00 & 5.15 \pm 0.06\end{array}$

Here the discrepancy observed for the B-value of the 00500 state is presumably due to the need for higher order terms caused by the large quantum number; that is, $\mathrm{B}^{\prime}=\mathrm{B}_{0}-\alpha_{3} \mathrm{v}_{3}-\beta_{3} \mathrm{v}_{3}^{2}$. If this is the case, our. data yield $\alpha_{3}=5.35 \times 10^{-3} \mathrm{~cm}^{-1}$ and $\beta_{3}=0.25 \times 10^{-3} \mathrm{~cm}^{-1}$. when combined with the values in Reference 3. This calculation does not yield very reliable values, however, and is given only to show the order of magnitude of the extra $\beta$-term required.

The discrepancy indicated by the $0-C$ value for 10300 is worthy of note. This band is influenced by an extremely strong rotational perturbation described by Funke (17). The constants given above were de- 
termined by fitting the observed line positions up to $\mathrm{J}=26$ to the formula discussed in this chapter. At about $J=26$ a crossing of states apparently takes place, with extra lines resulting; $J=18$ of the 10300 level is split into two levels $(R(17)$ and $P(19)$ of 10300 - 00000 are doublets), indicating a crossing of states at that point also.

With the exception of these two bands, all of the observed B values agree extremely well with those calculated using the $\alpha_{i}$ given in Reference 3.

The agreement of observed and calculated B-values for the other states indicates that the linear expression for this inertial coefficient remains good for the vast majority of levels which occur as upper states of transitions in the photographic region. It suggests that future evaluation of the $\alpha_{i}$ constants can include measured B-values from the photographic bands without the necessary inclusion of nonlinear terms in the expression used.

Most of the bands observable in the photographic region involve excitation of $v_{3}$ to $v=2$ or more and consequently require consideration of Darling-Dennison resonance as well as Fermi resonance of the form $\left(v_{3} \| v_{2}+v_{4}+v_{5}\right)$. Because of this, the vibrational calculations are complicated to a degree which makes them undesirable at this time. The band centers presented in this work, however, should aid in such calculations in the near future, when more levels have been determined from observed transitions. Most important, the vibrational calculations included here for the purposes of identifying the transitions to $1212^{0} 0$ and $1303^{1} 1^{1}$ gave such good results that it appears unlikely that any 
cubic vibrational terms need be included in the calculation of upper states for bands occurring throughout most of the photographic region; highly excited levels such as 00500 are probable exceptions.

The conclusion to be drawn from these observations is that it is not justifiable to use only data observed in the region beyond the photographic (3) in the determination of the best set of rotational and vibrational constants for ${ }^{12} \mathrm{C}_{2} \mathrm{H}_{2}$. Data from the visible and near infrared apparently satisfy the linear approximation for B-values and the quadratic vibrational expression for band centers, as long as resonance terms are properly included. Since this appears to be so, the inclusion of such data in future summaries, such as that given in 1963 in Reference 3, is strongly recommended and should aid in the numerical evaluation of off-diagonal elements, such as the Darling-Dennison resonance parameter. Additional studies of photographic bands involving $v_{1}$ and $v_{3}$ need to be made before this analysis can be performed with sufficient accuracy to justify the labor involved. Measurement of 10200,00400 and 40000 would help; this could be done, in principle, by observation of the transitions to these levels from the 00001 lower state. 


\section{APPENDIX}

The acetylene absorption bands observed in the three micron region:

$\begin{array}{ll}\text { Band A } & v_{3} \\ \text { Band B } & v_{2}+v_{4}^{1}+v_{5}^{1} \\ \text { Band C } & v_{3}+v_{4}^{1}-v_{4}^{1} \\ \text { Band D } & v_{3}\left(^{12} \mathrm{c}^{13} \mathrm{CH}_{2}\right) \\ \text { Band E } & v_{2}+2 v_{4}^{2}+v_{5}^{1}-v_{4}^{1} \\ \text { Band F } & v_{3}+v_{5}^{1}-v_{5}^{1} \\ \text { Band G } & v_{2}+v_{4}^{1}+2 v_{5}{ }^{1}-v_{5}{ }^{1} \\ \text { Band H } & v_{2}+v_{4}^{1}+v_{5}{ }^{1}\left({ }^{12} \mathrm{C}^{13} \mathrm{CH}_{2}\right)\end{array}$

The traces show the spectrum observed at different pressures. 


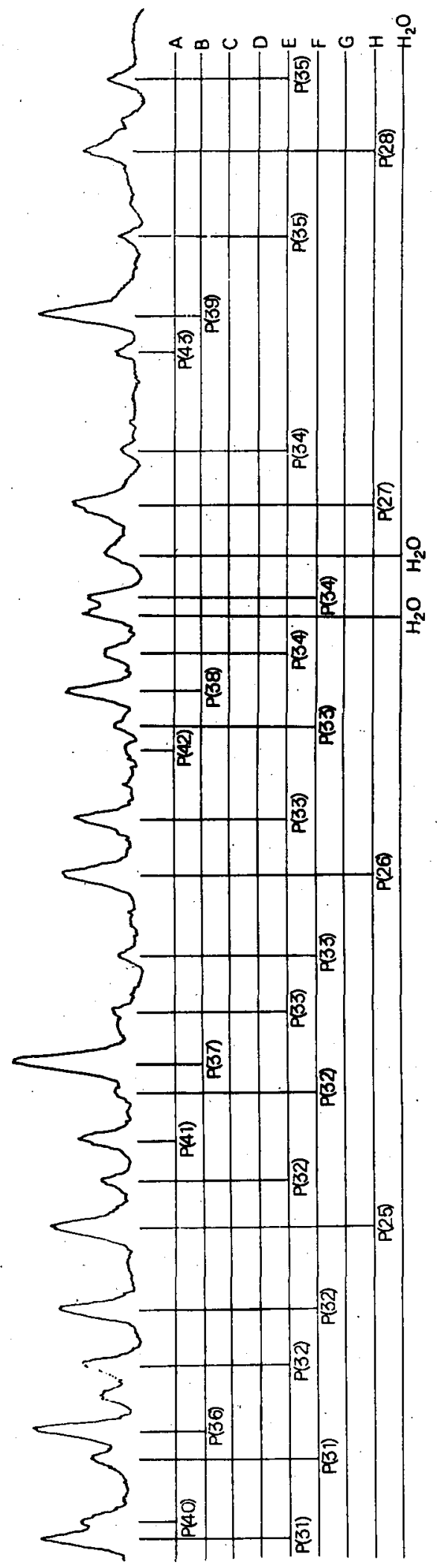




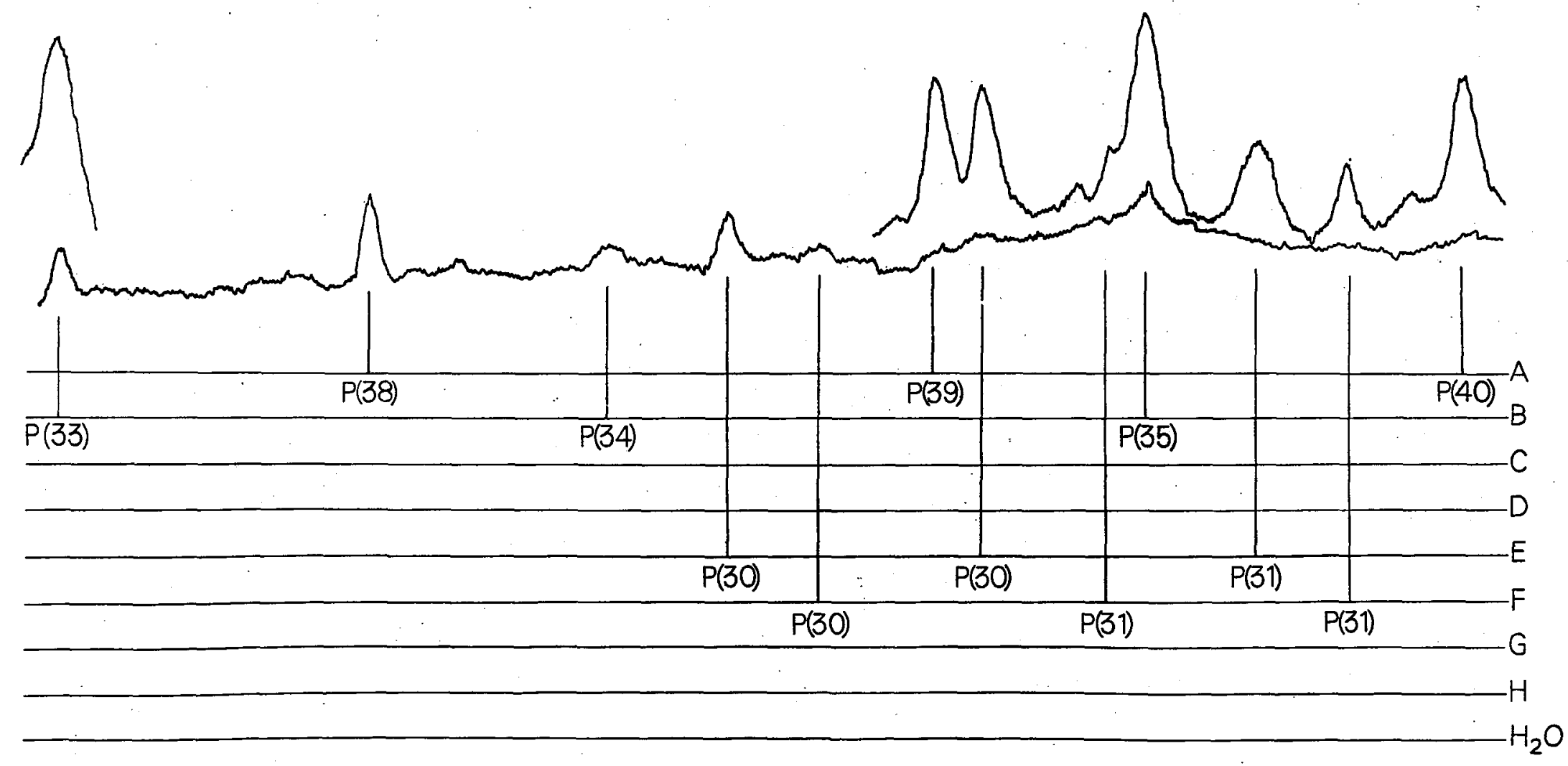




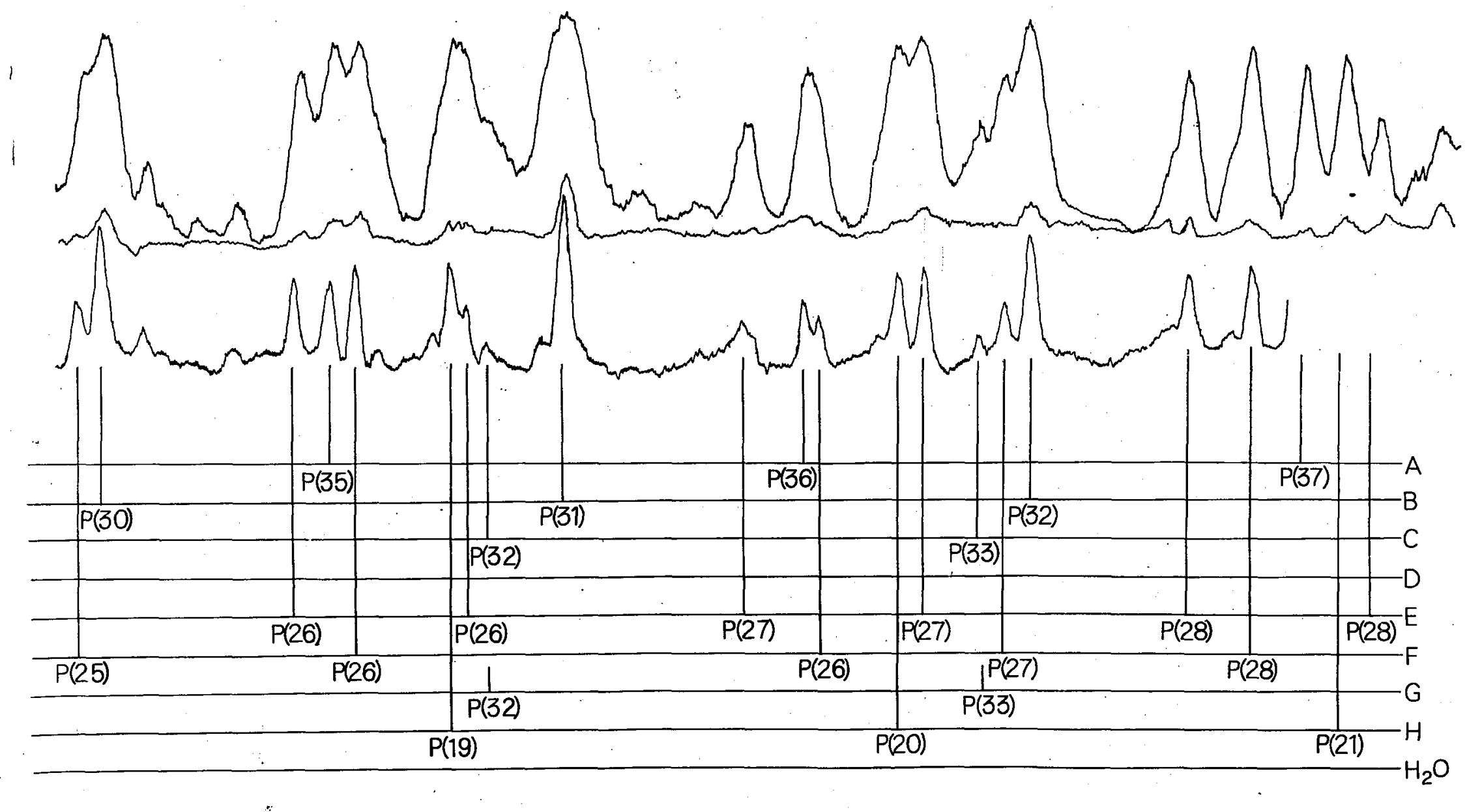




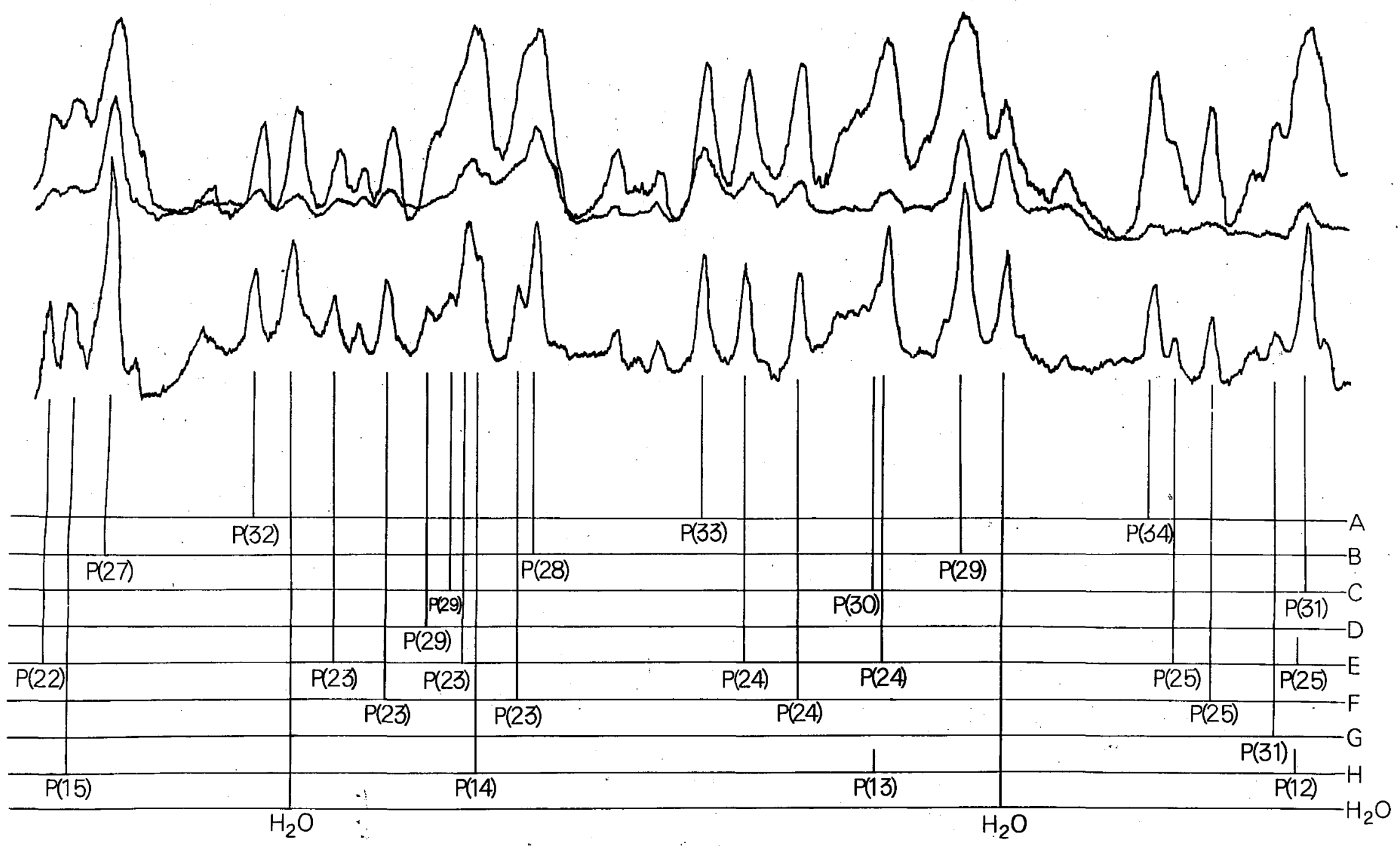

N 


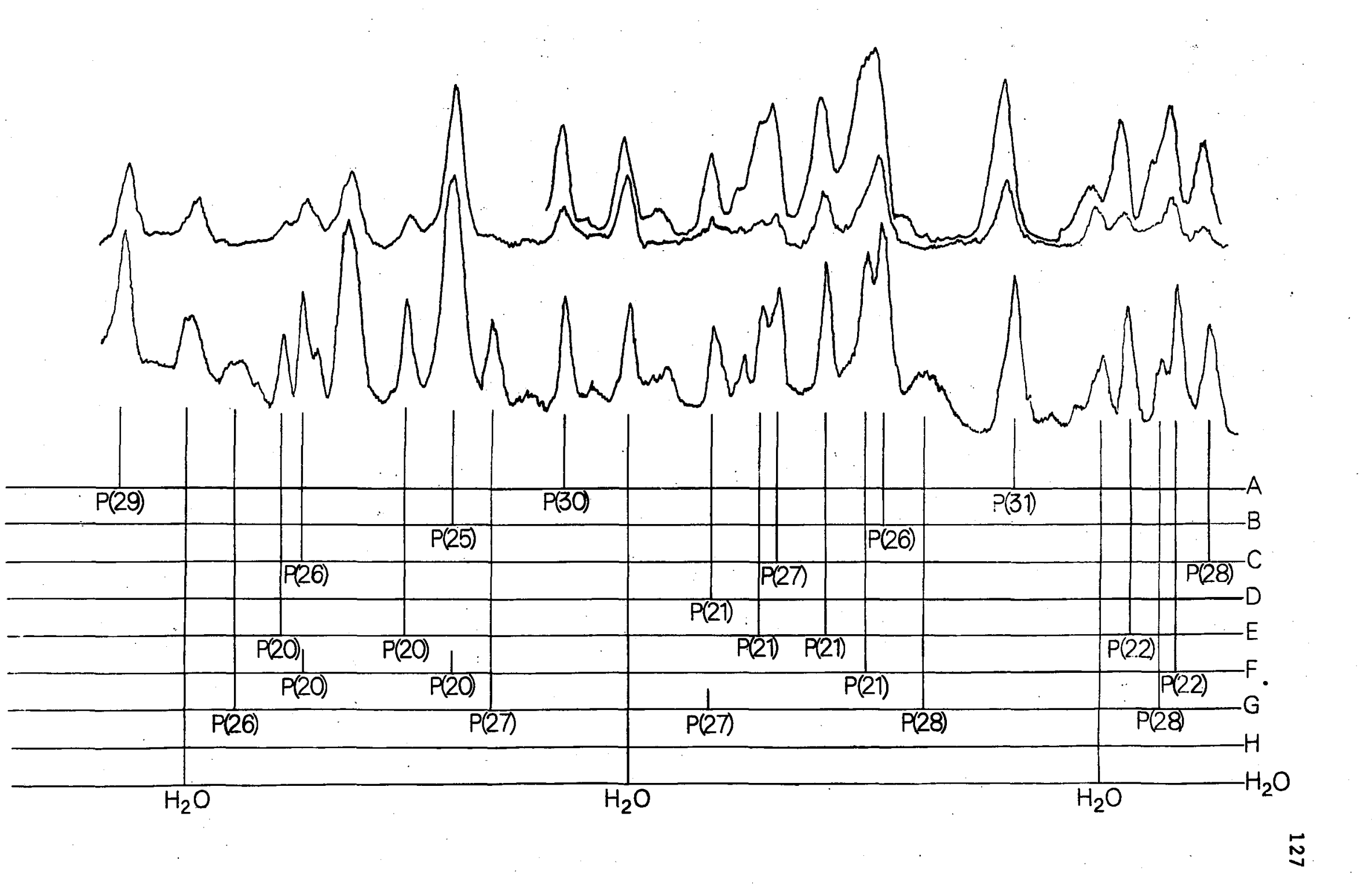




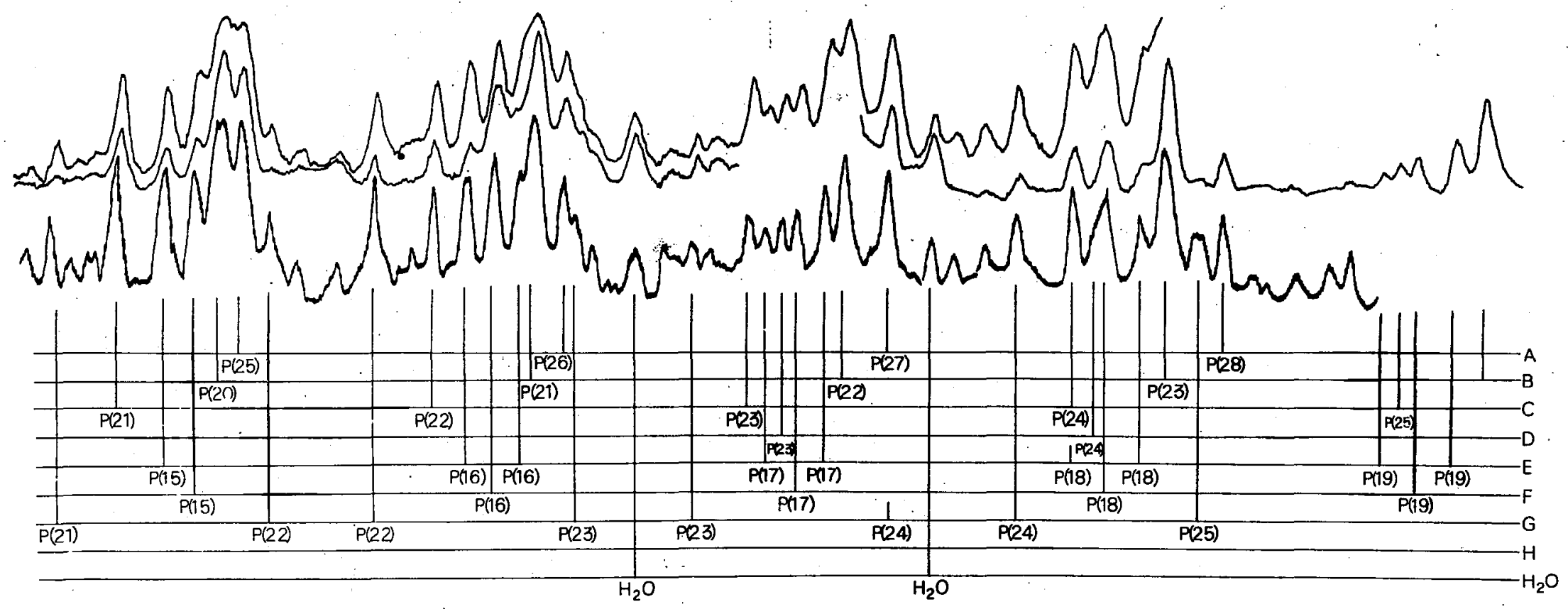

$\underset{\infty}{\mathbb{N}}$ 


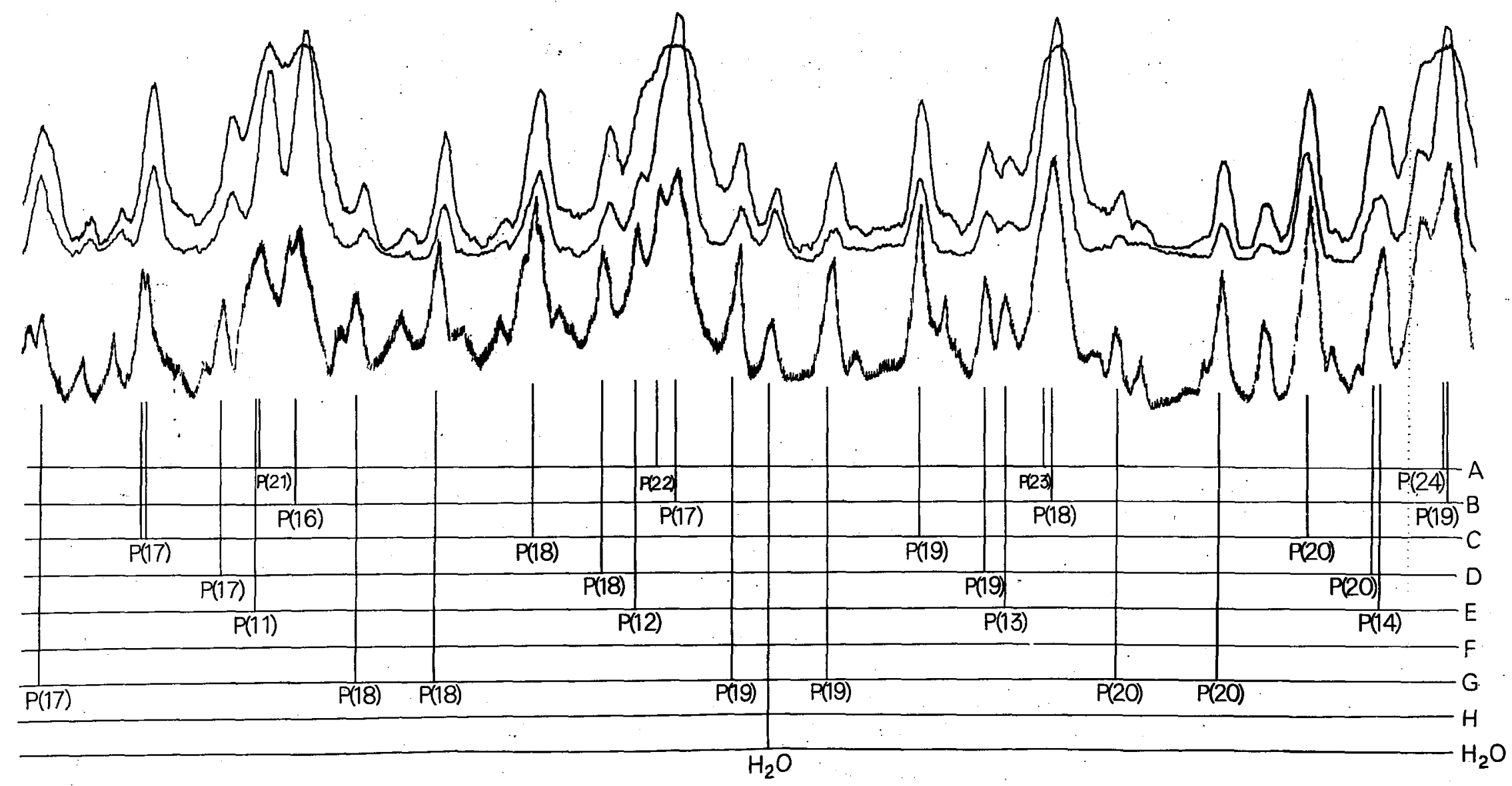


요

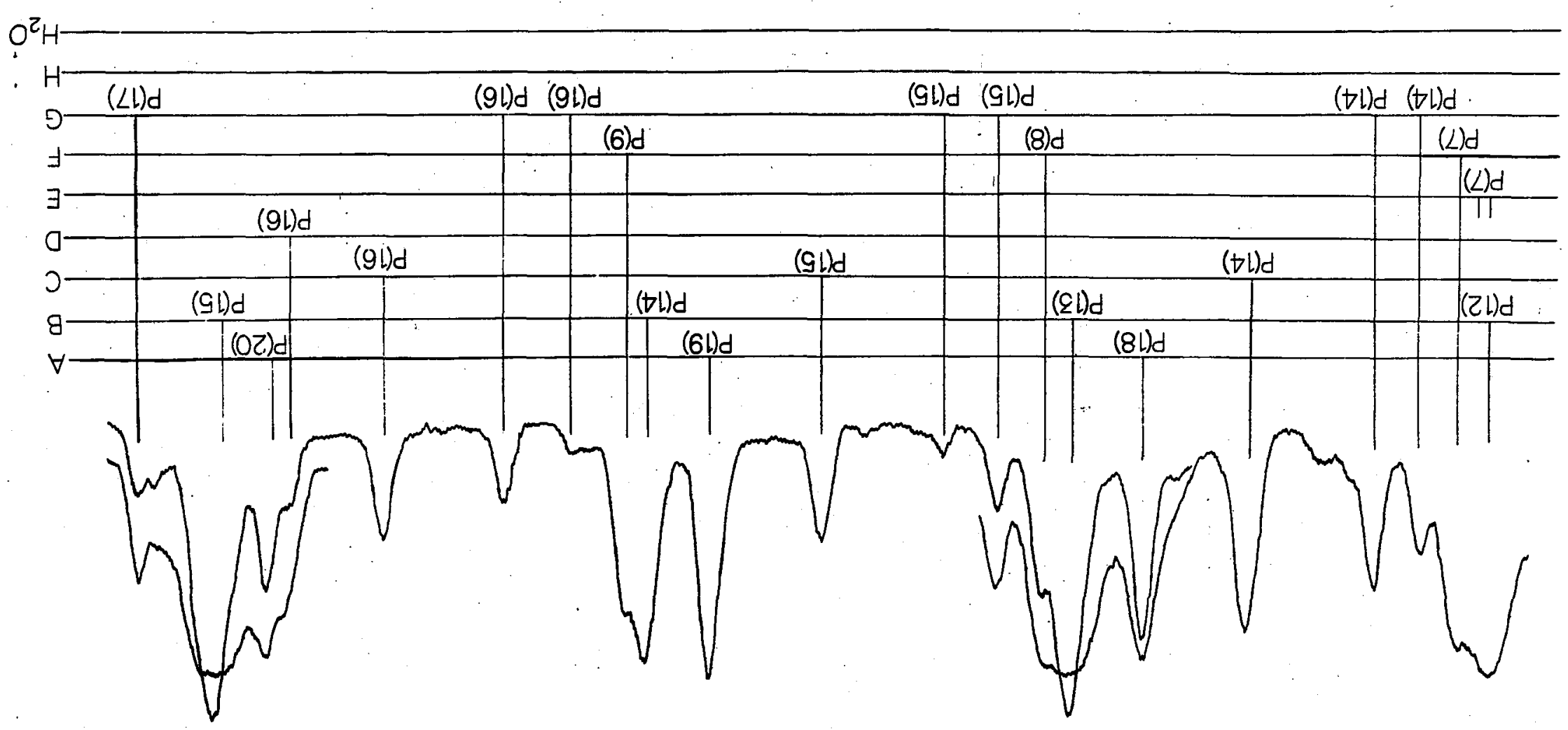




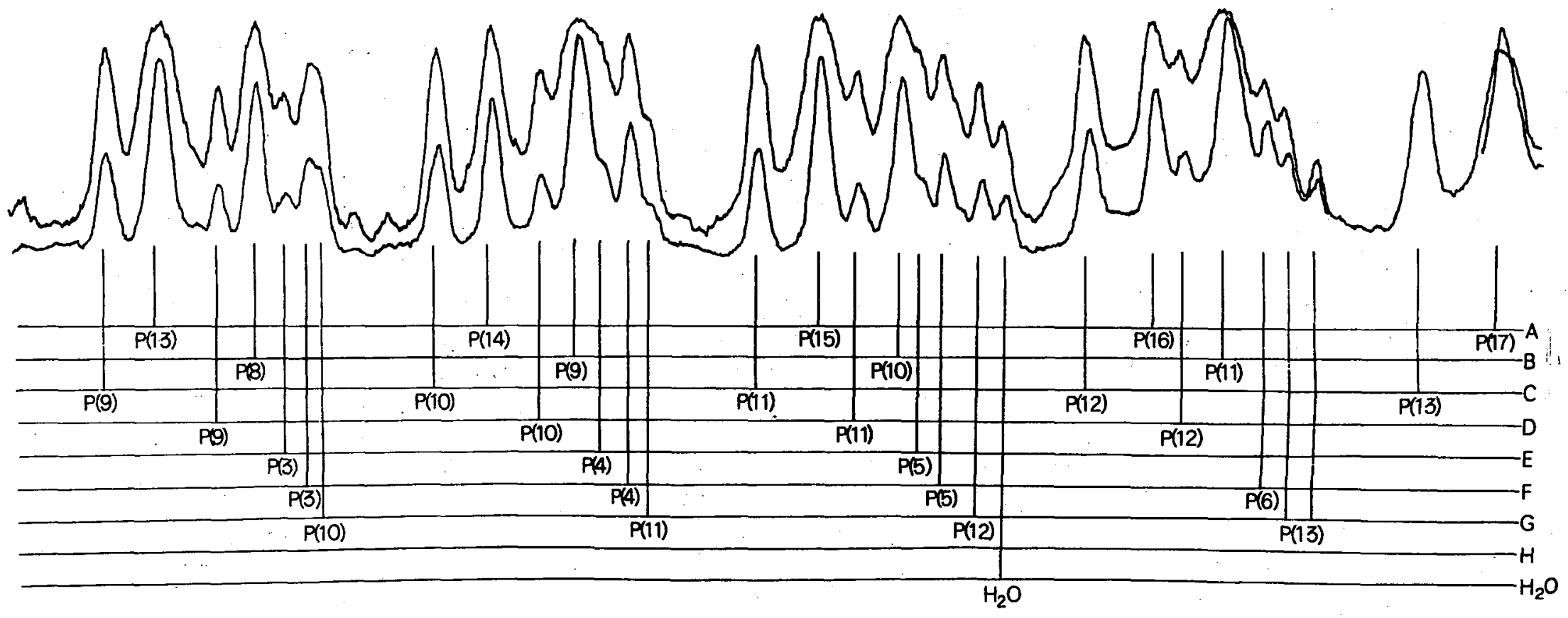

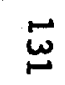




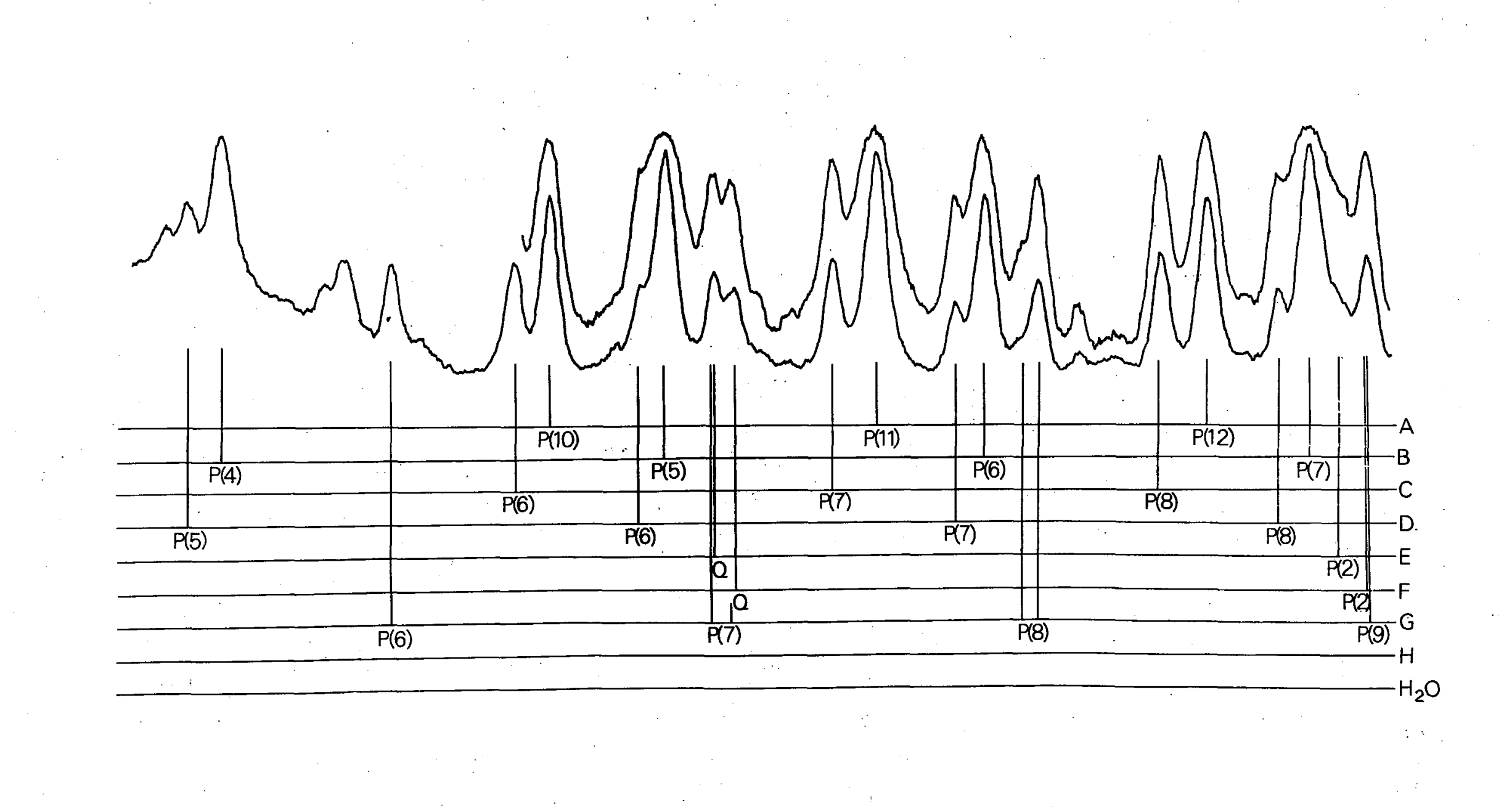




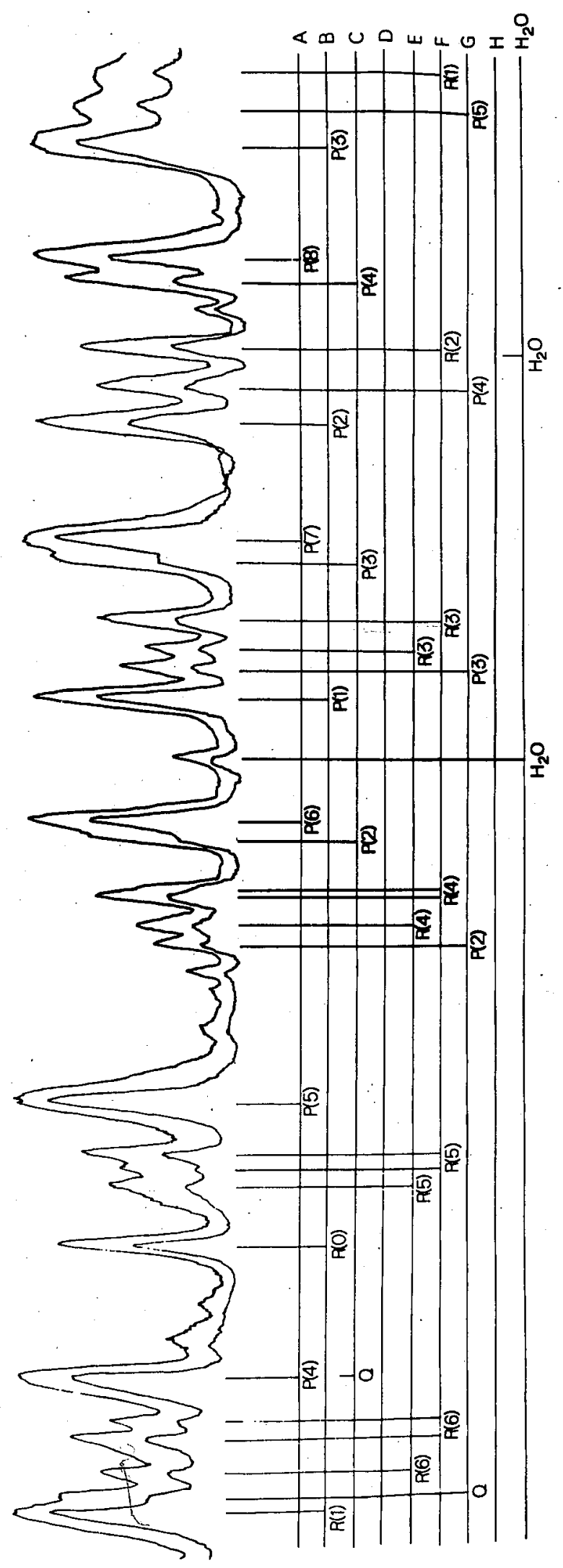




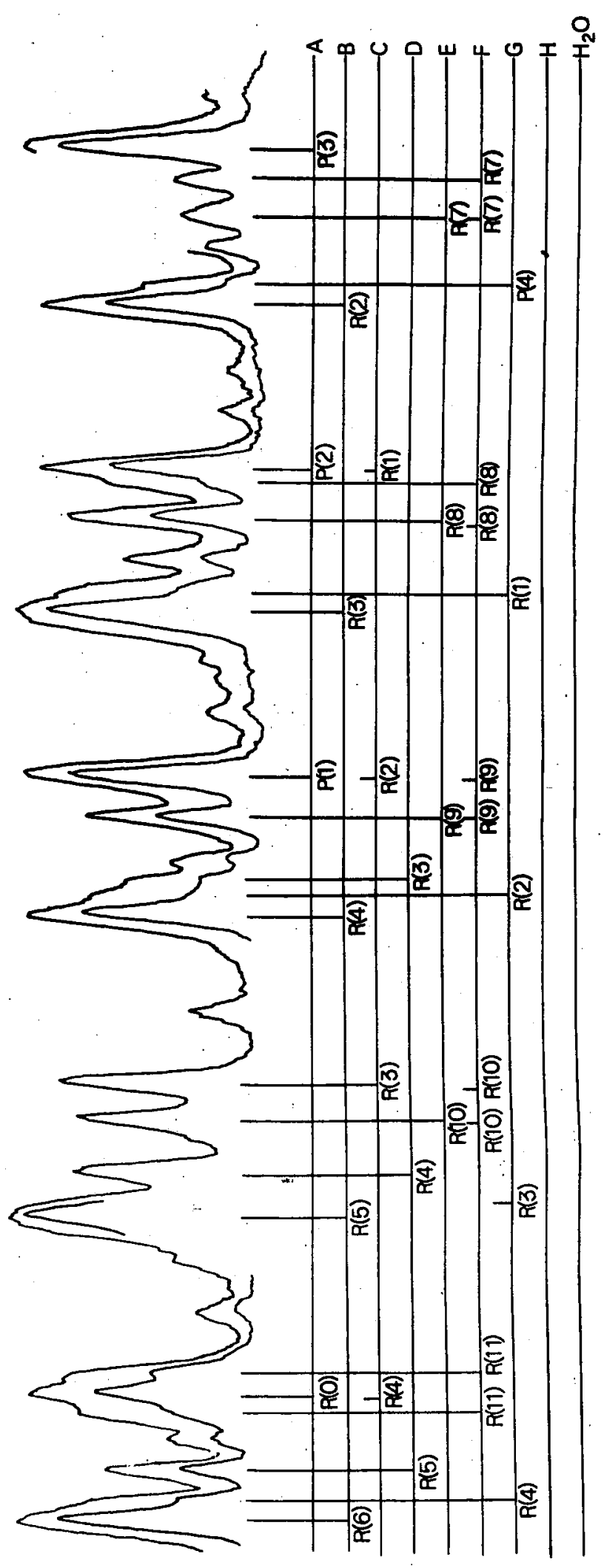

134 


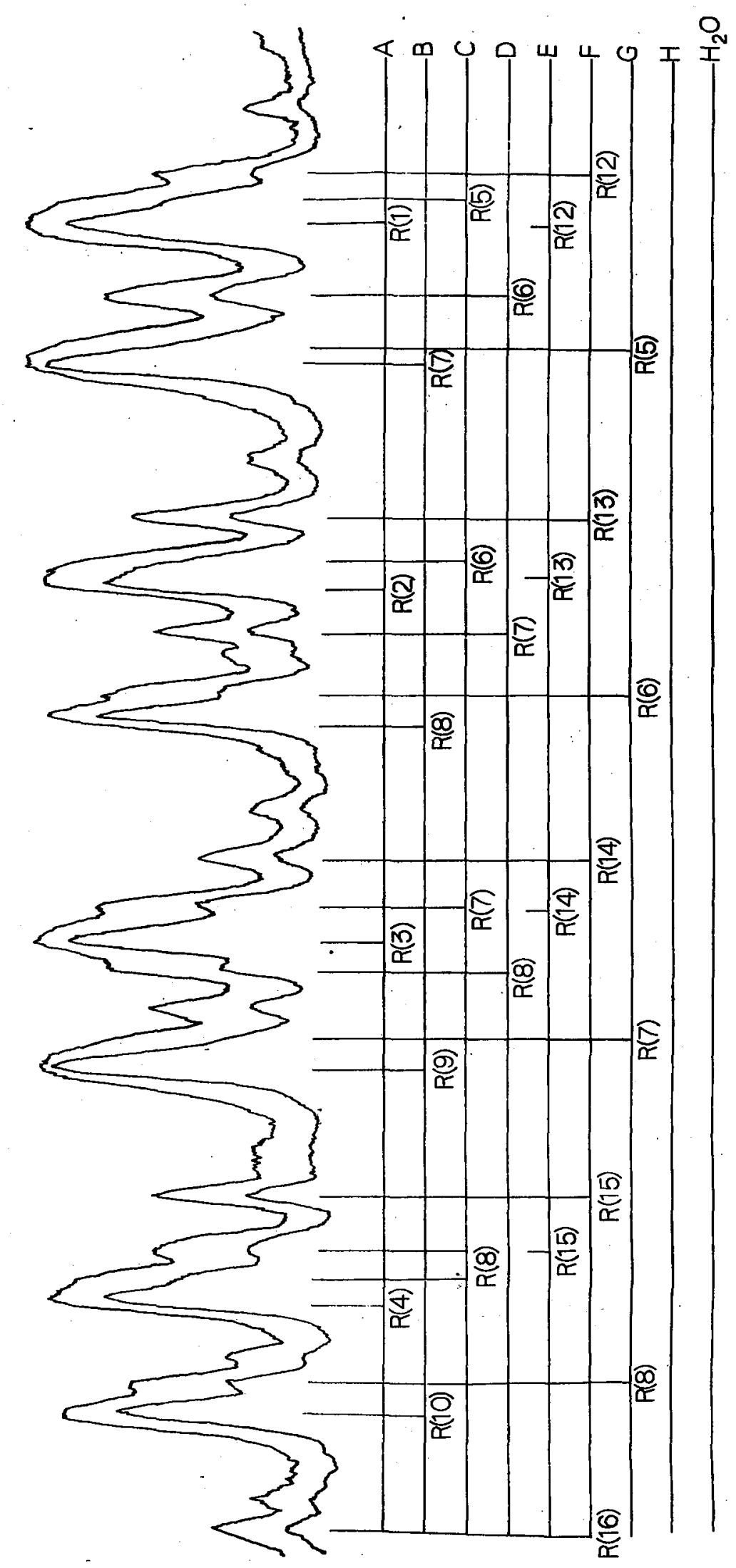




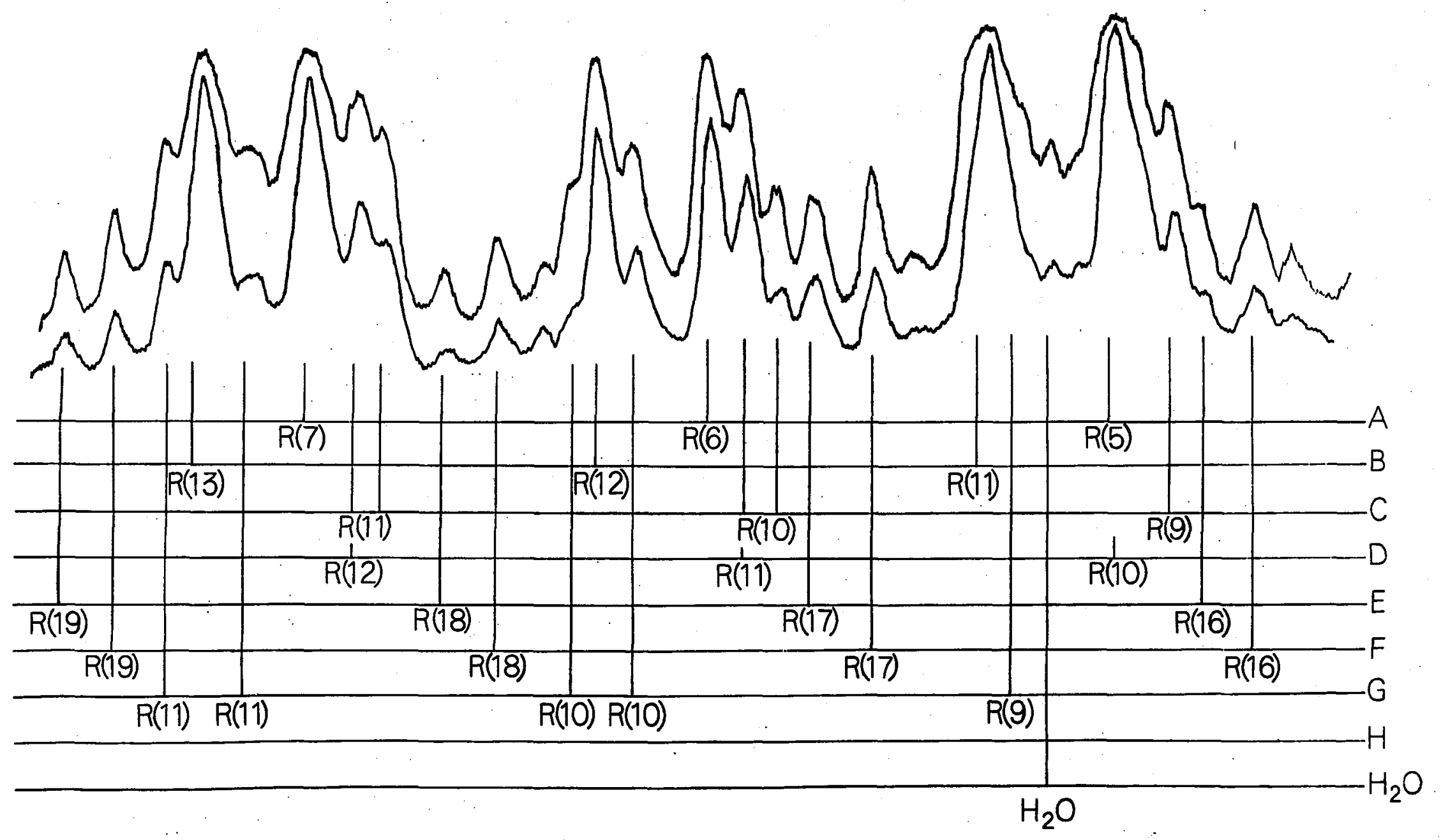

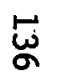




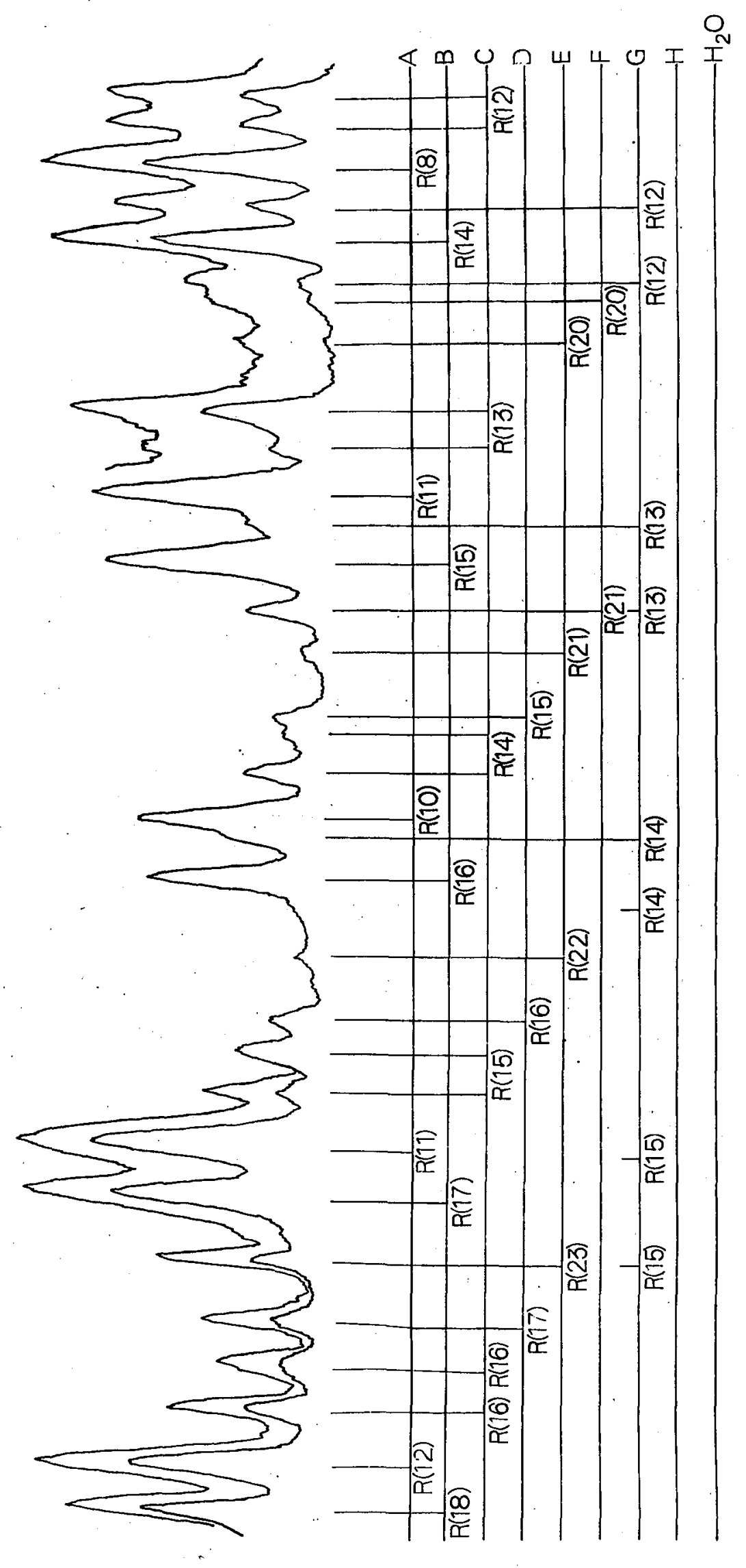




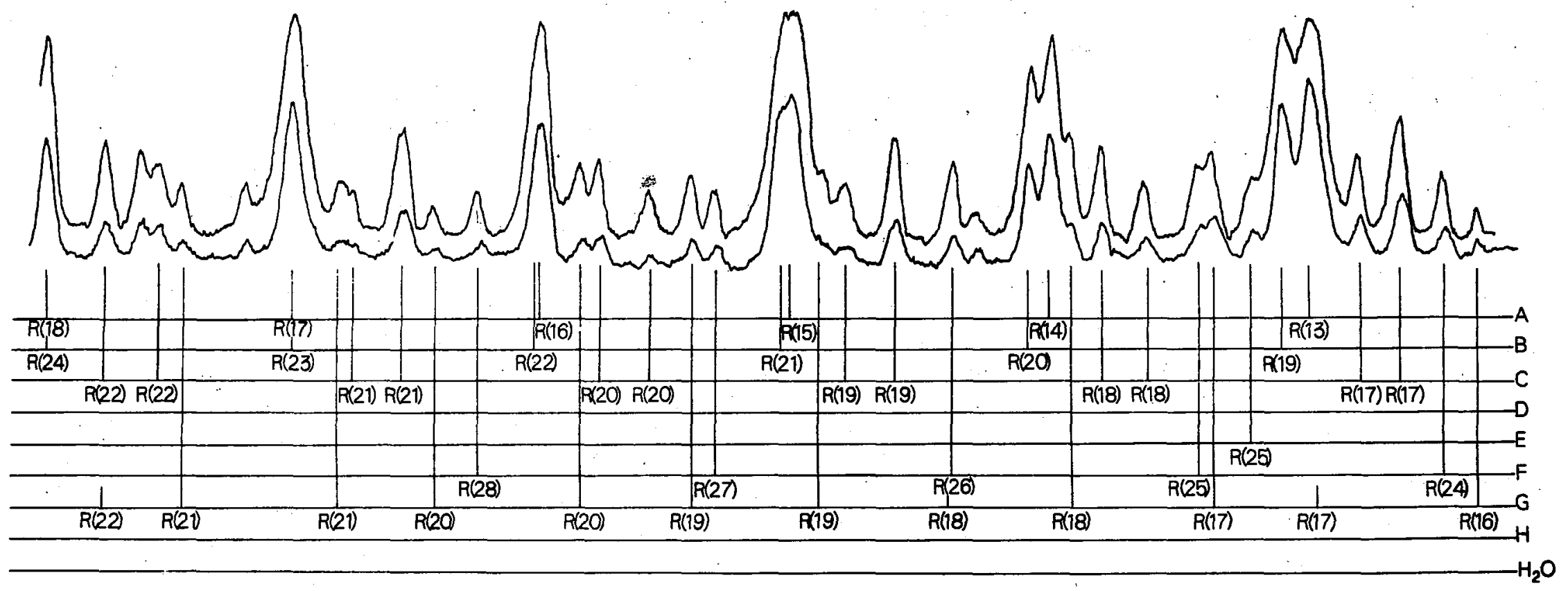

$\underset{\infty}{\omega}$ 


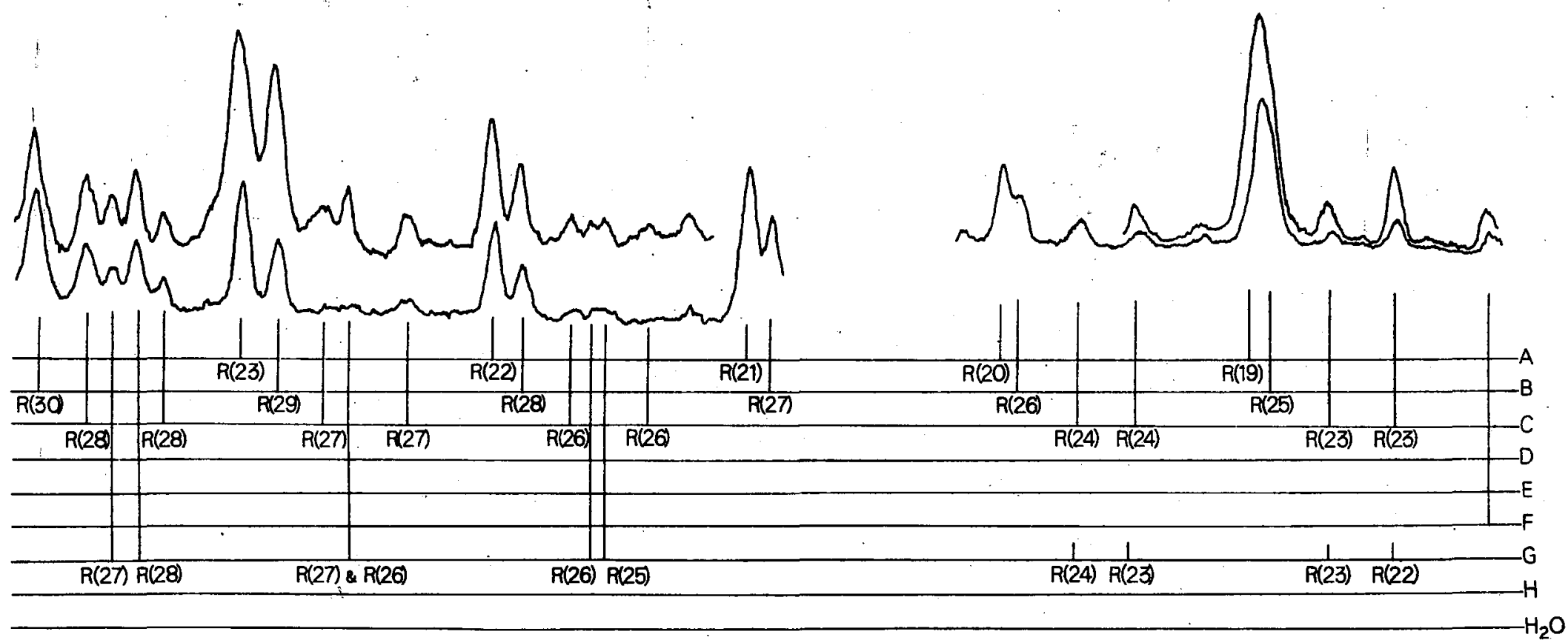




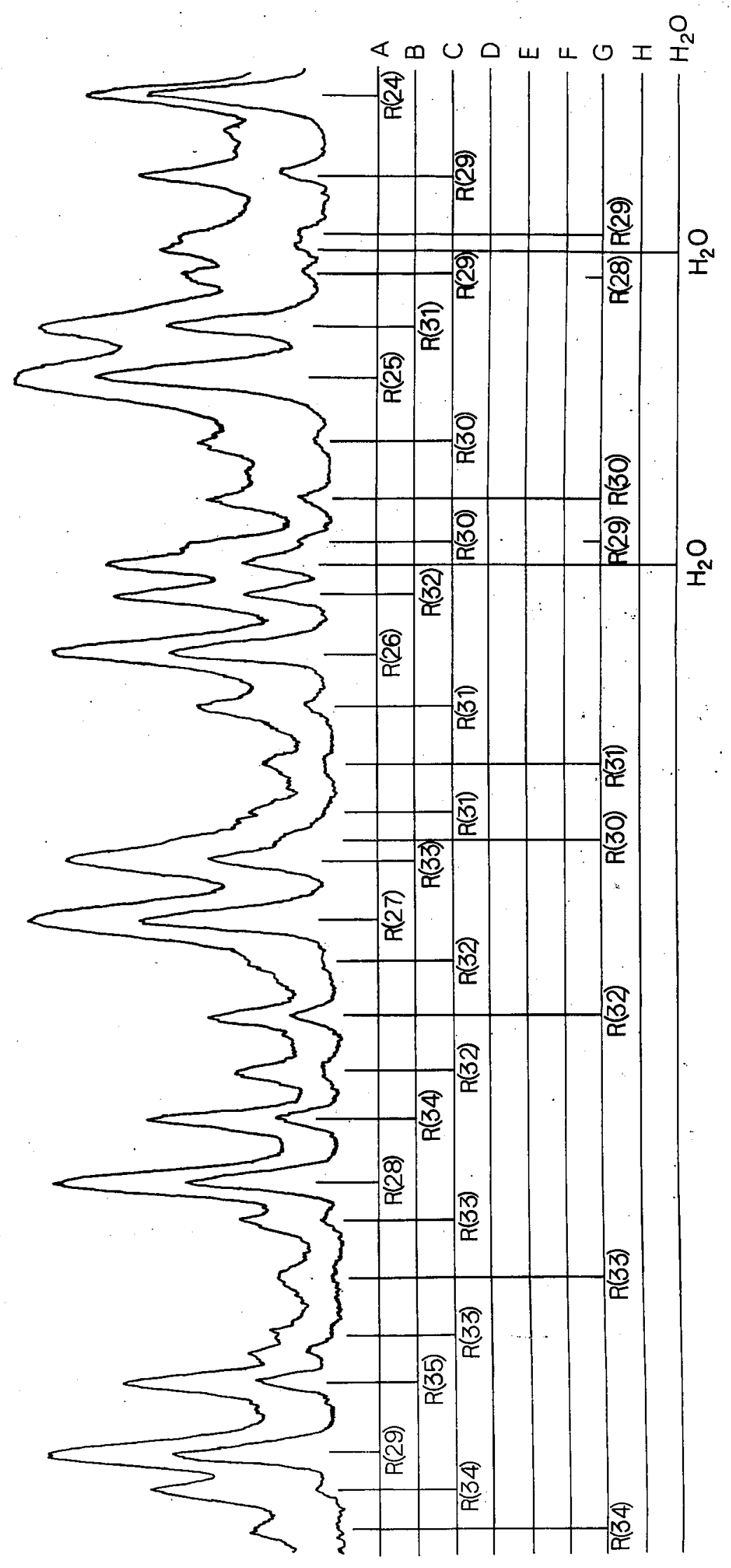




\section{REFERENCES}

1 G. Herzberg, "Molecular Spectra and Molecular Structure, Vol. II," Van Nostrand, Princeton, New Jersey, 1945.

2 W. J. Lafferty and R. J. Thibault, J. Mol. Spectry. 14, 79 (1964).

3 E. K. Plyler, E. D. Tidwell, and T. A. Wiggins, J. Opt. Soc. Am. 53, $589 \cdot(1963)$.

4 J. F. Scott and K. Narahari Rao, J. Mol. Spectry. 16, 15 (1965).

5 J. F. Scott and K. Narahari Rao, J. Mo1. Spectry. (in press).

6 G. Amat and H. H. Nielsen, J. Mol. Spectry. 2, 152 (1958).

7 F. L. Keller, Ph.D. dissertation, University of Tennessee, 1955.

8 W. H. Shaffer and A. H. Nielsen, J. Chem. Phys. 9, 847 (1941).

9 T. J. Coburn, K. Narahari Rao, and H. H. Nielsen, J. Chem. Phys. 25, 607 (1956).

10 G. Amat and H. H. Nielsen, J. Mol. Spectry. 2, 163 (1958).

11 H. H. Nielsen, G. Amat, and M. Goldsmith, J. Chem. Phys. 26, 1060 (1957).

12 T. A. Wiggins, E. K. Plyler, and E. D. Tidwe11, J. Opt. Soc. Am. 51, 1219 (1961).

13 H. H. Nielsen and W. H. Shaffer, J. Chem. Phys. 11, 140 (1943).

14 K. K. Innes, J. Chem. Phys. 22 , 863 (1954).

15 J. F. Scott and K. Narahari Rao, J. Mo1. Spectry. 18, 152 (1965).

16 J. F. Scott and K. Narahari Rao, J. Mo1. Spectry. 18, 451 (i965).

17 F. W. Loomis and R. W. Wood, Phys. Rev. 32, 223 (1928).

18 G. W. Funke, Zeits. f. Physik 104, 169 (1936). 